\title{
Hidden diversity within the Nemastoma bidentatum Roewer, 1914 complex (Opiliones: Nemastomatidae) Part I: Morphological evidence
}

\author{
Tone NOVAK ${ }^{\circledR 1, *}$, Ljuba SLANA NOVAK ${ }^{\circledR 2}$, Peter KOZEL ${ }^{\circledR 3}$, \\ Miriam Gudrun SCHAIDER ${ }^{\circledR 4}$, Christian KOMPOSCH ${ }^{\circledR 5}$, Saška LIPOVŠEK ${ }^{\circledR 6}$, \\ Jan PODLESNIK ${ }^{\circledR 7}$, Igor PAUŠIČ ${ }^{\circledR 8}$ \& Günther RASPOTNIG ${ }^{\circledR 9}$ \\ 1,3,6,7,8 Department of Biology, Faculty of Natural Sciences and Mathematics, \\ University of Maribor, Koroška 160, 2000 Maribor, Slovenia. \\ ${ }^{2}$ Ozare 31, 2380 Slovenj Gradec, Slovenia. \\ ${ }^{3}$ Karst Research Institute IZRK ZRC SAZU, Titov trg 2, 6230 Postojna, Slovenia. \\ ${ }^{3}$ UNESCO Chair on Karst Education, University of Nova Gorica, Vipava, Slovenia. \\ ${ }^{4,9}$ Institute of Biology, Karl-Franzens University, Universitätsplatz 2, 8010 Graz, Austria. \\ ${ }^{5}$ ÖKOTEAM - Institut für Tierökologie und Naturraumplanung, Institute for Animal Ecology and \\ Landscape Planning, Bergmanngasse 22, 8010 Graz, Austria. \\ ${ }^{6}$ Faculty of Medicine, University of Maribor, Taborska ulica 8, 2000 Maribor, Slovenia. \\ ${ }^{6}$ Faculty of Chemistry and Chemical Engineering, University of Maribor, \\ Smetanova ulica 17, 2000 Maribor, Slovenia. \\ ${ }^{6}$ Gottfried Schatz Research Center, Division of Cell Biology, Histology and Embryology, \\ Medical University of Graz, Neue Stiftingtalstrasse 6, 8010 Graz, Austria. \\ *Corresponding author: tone.novak@guest.um.si \\ 2Email: tone.novak@guest.arnes.si \\ 33Email: peter.kozel@um.si \\ ${ }^{4}$ Email: miriamschaider@hotmail.com \\ ${ }^{5}$ Email: c.komposch@oekoteam.at \\ ${ }^{6}$ Email: saska.lipovsek@um.si \\ ${ }^{7}$ Email: jan.podlesnik@um.si \\ ${ }^{8}$ Email: igor.pausic@um.si \\ ${ }^{9}$ Email: guenther.raspotnig@uni-graz.at

\footnotetext{
${ }^{1}$ urn:lsid:zoobank.org:author:F6B51342-4296-473E-A3E8-AB87B0655F49

${ }^{2}$ urn:Isid:zoobank.org:author:1603E85F-EE29-475A-8F98-B8B8E059C030

${ }^{3}$ urn:1sid:zoobank.org:author:8C33627F-2D3D-4723-A059-B87462535460

${ }^{4}$ urn:lsid:zoobank.org:author:8787CDCA-1F3E-48A2-8139-5CB73DE71733

${ }^{5}$ urn:lsid:zoobank.org:author:CDE15756-4605-4C5C-AE6C-C576852C9092

${ }^{6}$ urn:1sid:zoobank.org:author:1F7DB78E-CEE0-4840-95B4-8E4ADA3211DB

${ }^{7}$ urn:lsid:zoobank.org:author:59572EBE-8871-4EAC-92F7-F87201CB6F67

${ }^{8}$ urn:lsid:zoobank.org:author:35939273-4DBF-4CC4-AB06-FBDF1D17051B

${ }^{9}$ urn:lsid:zoobank.org:author:75E9550E-01EF-439F-A3AB-6F463F35D03B
}

Dedicated to our teachers, colleagues and friends Dr Jürgen Gruber and Em. Prof. Dr Jochen Martens. 
Abstract. Nemastoma bidentatum Roewer, 1914 is a complex of closely related litter-dwelling harvestmen, characterized by a penis glans with two pairs of lateral lancet-like spines, and a femur IV with a sawlike series of pointed tubercles. Here we a) revise the hitherto known taxa within the $N$. bidentatum complex, and b) analyze in detail the relations among the taxa in Slovenia. The study revealed that the $N$. bidentatum complex consists of four species: $N$. bidentatum Roewer, 1914 s. str., $N$. relictum Gruber \& Martens, 1968 stat. nov., N. pluridentatum (Hadži, 1973) stat. nov. and N. kozari Novak, Kozel, Podlesnik \& Raspotnig sp. nov. Moreover, $N$. bidentatum s. str. consists of six subspecies: $N$. bidentatum bidentatum Roewer, 1914, N. bidentatum sparsum Gruber \& Martens, 1968, N. bidentatum gruberi Novak, Slana Novak, Kozel \& Raspotnig ssp. nov., N. bidentatum martensi Novak, Slana Novak \& Raspotnig ssp. nov., N. bidentatum schmidti Novak, Raspotnig \& Slana Novak ssp. nov. and N. bidentatum sneznikensis Novak, Komposch, Slana Novak \& Raspotnig ssp. nov. In Slovenia, the six subspecies of $N$. bidentatum form a parapatric complex around $N$. bidentatum schmidti. Hybrids occur in the contact zones between adjacent subspecies, but they are missing between distant subspecies. The taxonomic distinction of lineages/subspecies is congruent with their distribution patterns. With six of the nine taxa present, Slovenia is considered the center of the $N$. bidentatum complex speciation. At the time being, this complex is the most diversified harvestman group on a subspecific and young-species level and provides important details on speciation processes in Opiliones.

Keywords. Allopatric speciation, arachnids, Dyspnoi, morphological taxonomy, parapatric speciation.

Novak T., Slana Novak L., Kozel P., Schaider M.G., Komposch C., Lipovšek S., Podlesnik J., Paušič I. \& Raspotnig G. 2021. Hidden diversity within the Nemastoma bidentatum Roewer, 1914 complex (Opiliones: Nemastomatidae) Part I: Morphological evidence. European Journal of Taxonomy 777: 1-67.

https://doi.org/10.5852/ejt.2021.777.1561

\section{Introduction}

In recent decades, relations among the higher taxa of harvestmen have become increasingly clear and amenable (Schönhofer 2013; Giribet \& Sharma 2015; Fernández et al. 2017). However, there is still a need for major revisions in some taxa at the genus and family levels. Dyspnoi Hansen \& Sørensen, 1904, e.g., still exhibit many problems with species delineation and assignment (Schönhofer 2013). Except for some Ortholasmatinae Shear \& Gruber, 1983, Dyspnoi are restricted to the temperate zones (Gruber 2007). In central Europe, more than a third of harvestman species and subspecies belong to this suborder (Blick \& Komposch 2004), while in Slovenia they account for almost half (Novak et al. 2006; unpublished own data). One unresolved puzzle within Dyspnoi is the Nemastoma bidentatum Roewer, 1914 complex, Nemastomatidae Simon, 1872, distributed in Central and Eastern Europe, with sparse, disjunct localities in northern Europe. These 1.8-2.5 mm long, black or nearly black Nemastoma (Fig. 1), have been recorded in Austria, Bosnia and Herzegovina, Bulgaria, Croatia, Czech Republic, Germany, Hungary, Italy, Montenegro, Romania, Russia, Serbia, Slovakia, Slovenia and Turkey (Roewer 1914, 1923, 1951; Gruber \& Martens 1968; Hadži 1973a; Staręga 1976; Martens 1978, 2006; Mučalica 1988; Ćurčić et al. 1999; Klimeš 2000; Novak \& Gruber 2000; Blick \& Komposch 2004; Komposch \& Gruber 2004; Novak 2004a, 2004b; Babalean 2005; Novak et al. 2006; Schönhofer \& Holle 2007; Komposch 2009, 2011; Schönhofer 2013; Kurt 2014).

Roewer $(1914,1923)$ cited Comana Vlasca in Romania as the type locality of $N$. bidentatum Roewer, 1914. Hadži (1927) provided the first detailed drawings of $N$. bidentatum from an unknown locality in Slovenia, deposited in the Ferdinand Schmidt Collection at the Natural History Museum in Ljubljana. Gruber \& Martens (1968) established that Roewer's illustrations show a taxon living exclusively in the Eastern Alps. Accordingly, they (ibid.) redescribed the nominal subspecies N. b. bidentatum Roewer, 1914 upon individuals from Bärental in the Karawanken Mts, Carinthia, Austria, along with two new 

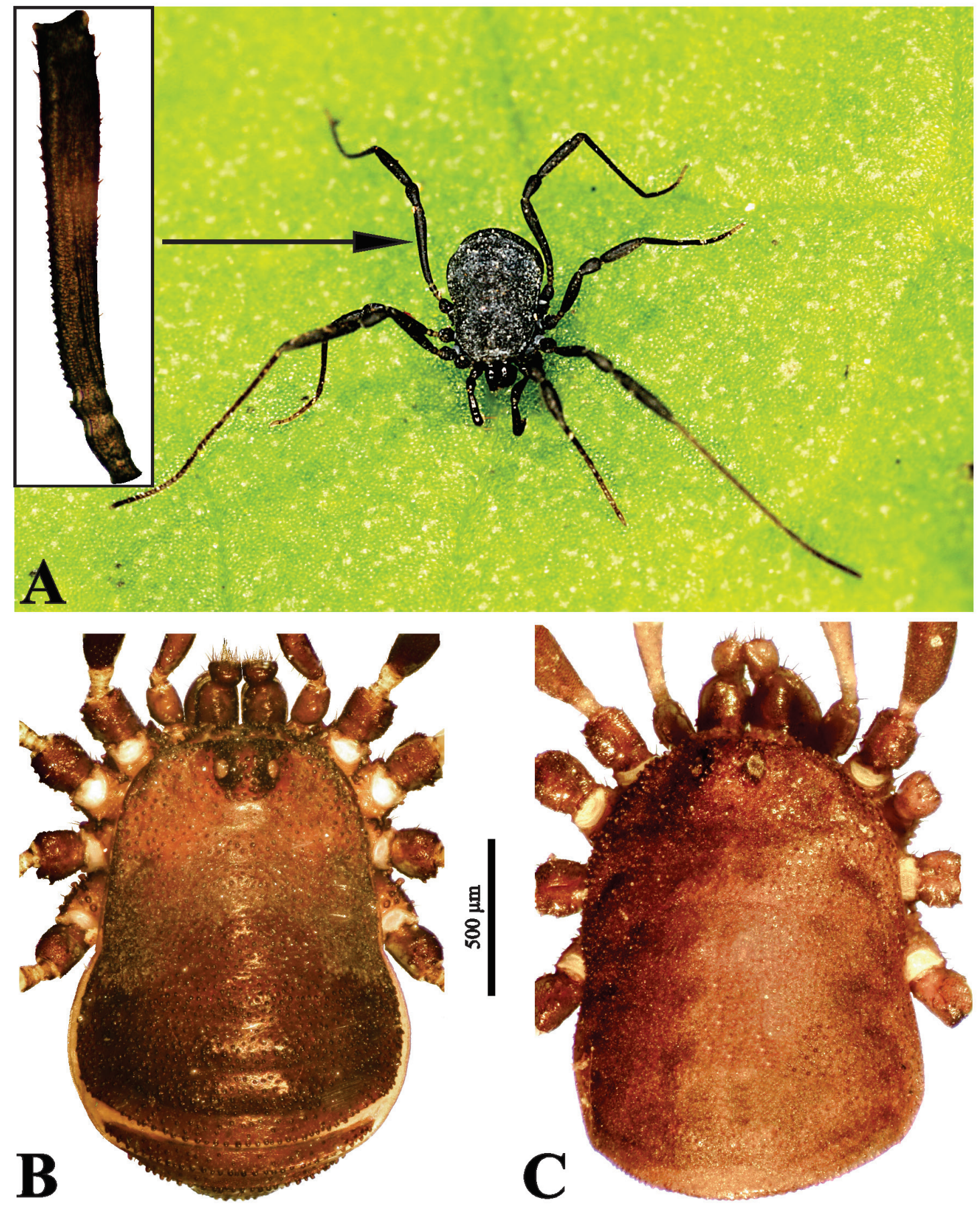

Fig. 1. Representatives of the Nemastoma bidentatum Roewer, 1914 complex. A. The habitus of a male Nemastoma bidentatum gruberi Novak, Slana Novak, Kozel \& Raspotnig ssp. nov.; Svete gore, Slovenia (photo: C. Komposch); inset: femur IV with two pseudo-articles (apparent articles, i.e., sections delimited with pale, indented rings on the proximal femur portion), and a saw on the latero-posterior margin. $\mathbf{B}$. The body of a male Nemastoma relictum Gruber \& Martens, 1968 stat. nov. showing the tuberculate surface of glittering scutum compositum, and position of the ocular mound (tuber oculorum), supracheliceral lamellae, chelicerae with cheliceral apophysis, and pedipalps and leg. The frontal portion of the scutum is smeared over with a hardened secretum of the integumental glands (cf. Wolff et al. 2016); Mt. Großes Karr, the Koralpen Mts, Austria. C. The body of a male Nemastoma bidentatum gruberi ssp. nov., smeared over with the secretum; Kozje, Slovenia. 
subspecies: N. b. sparsum Gruber \& Martens, 1968 and N. b. relictum Gruber \& Martens, 1968. They ascribed the subspecies rank to the new taxa, since there were only limited differences in the genital morphology, and hybrids between $N$. b. bidentatum and $N . b$. sparsum probably occurred. They (ibid.) also reported $N$. b. bidentatum and $N$. $b$. sparsum from Slovenia. Later, Hadži (1973a) described two new taxa belonging to the $N$. b. complex: Nemastoma (Lugubrostoma) triste pluridentatum Hadži, 1973 and Nemastoma (Stridulostoma) seliskari Hadži, 1973. Gruber (1976) synonymized Stridulostoma Hadži, 1973 with Nemastoma C.L. Koch, 1836, and Martens (1978) synonymized both taxa with N. b. bidentatum. Novak (2005a) substantiated $N$. ?bidentatum pluridentatum (the question mark denotes the unresolved subspecific/specific status) from Bosnia as a valid taxon.

Gruber \& Martens (1968) explained that, in Austria, N. b. bidentatum and N. b. relictum do not interbreed in syntopy, and, accordingly, $N$. $b$. relictum should be raised to the species level if no hybrids were found in the future. Furthermore, Martens (1978) anticipated the syntopic occurrence of $N$. b. bidentatum and $N$. b. sparsum without hybridization. This was later confirmed in Austria, where $N . b$. bidentatum, $N$. b. relictum and $N$. b. sparsum behave as bona fide species (Komposch \& Gruber 2004; Komposch 2009). Hybrids between $N$. b. bidentatum and $N$. b. sparsum have been mentioned in Slovenia and Croatia (Gruber \& Martens 1968; Martens 1978).

The subspecies category was developed to enhance understanding of geographic variation and speciation, and to refine taxonomic distinction (Mallet 2013; Torstrom et al. 2014). There is still much disagreement on this issue, calling for the taxonomic clarification of diagnosability at the subspecies rank level (Patten \& Unitt 2002; Isaac et al. 2004; Haig et al. 2006; Sangster 2009). Genetic techniques, e.g., altered the rules for subspecific designation by applying a phylogeny-based approach, but this should not be to the exclusion of other, traditional lines of evidence; instead, an integrative taxonomic approach, providing a more objective method, has been suggested (Torstrom et al. 2014).

Taxa described as subspecies must exhibit their own evolutionary trajectory and not be clinal variants based on a single characteristic (Zink 2004; Oatley et al. 2011). When describing new subspecies or revising subspecies taxonomy, detailed description of the concepts and criteria used to determine taxonomic relations should accompany each proposed classification or reclassification (Haig et al. 2006). Higher levels of confidence can be obtained by the inclusion of multiple independent sets of characters, such as from morphology, molecular genetics, ecology, behavior and/or physiology (Haig et al. 2006; De Queiroz 2007; Miralles et al. 2010; Braby et al. 2012).

In Dyspnoi, subspecies have been described for seven of the 36 hitherto recognized genera (Schönhofer 2013). Within Nemastomatidae, most subspecies are well defined, while others, e.g., those of Mitostoma olgae (Šilhavý, 1939) deserve revision (Novak 2004b; Schönhofer 2013). To date, in the genus Nemastoma C.L. Koch, 1836, seven valid species and four subspecies are known (Gruber \& Martens 1968; Hadži 1973a; Novak 2005a; Schönhofer 2013). These species are N. bidentatum Roewer, 1914, N. bimaculatum (Fabricius, 1775), N. dentigerum Canestrini, 1873, N. lugubre (Müller, 1776), N. schuelleri Gruber \& Martens, 1968, N. transsylvanicum Gruber \& Martens, 1968 and N. triste (C.L. Koch, 1835). All four subspecies ranked within Nemastoma bidentatum Roewer, 1914: N. b. bidentatum Roewer, 1914, N. ?b. pluridentatum (Hadži, 1973), N. b. relictum Gruber \& Martens, 1968 and N. b. sparsum Gruber \& Martens, 1968.

Genetic analyses of Nemastomatidae are still scarce, currently providing no reliable model to evaluate relations at the subspecies level (Schönhofer \& Holle 2007). Fortunately, chemical analysis of the opilionid scent gland products, which comprise a variety of chemicals, contribute significantly to disentangling specific profiles from higher taxa to the subspecies level (Raspotnig et al. 2014). Despite the reluctance of many Dyspnoi to release scent gland substances (Raspotnig et al. 2014), the Nemastomatidae appear 
to produce easily collectable and highly specific secretions that are very suitable as characters for (chemo) taxonomy (Raspotnig et al. 2017). In Paranemastoma quadripunctatum Perty, 1833, Raspotnig et al. (2010) discovered an exclusively quinonic mixture of seven substances. The secretions of Nemastoma triste contained a bouquet of 10 compounds, including quinones and acyclic ketones (Schaider et al. 2018).

The taxonomic problems within the $N . b$. complex deserve renewed consideration of the taxa involved. In Slovenia, a systematic collection over several years has provided comprehensive morphological, zoogeographical, chemical and genetic data that allow new insights into the species/subspecies relations in Nemastoma. Most of the unresolved questions refer to populations in Slovenia where a number of individuals of the $N$. $b$. complex cannot be assigned to any of the currently evidenced subspecies: N. b. bidentatum and N. b. sparsum.

In this revision, we combined morphological, geographical and ecological data. Genetic and chemical data from scent gland secretions were compiled for all species and subspecies of the genus Nemastoma and deserve a separate publication. The aims of this contribution are as follows: a) to revise recent knowledge on the $N$. $b$. complex and b) to analyze relations among the taxa of the complex on the territory of Slovenia in greater detail.

\section{Material and methods}

\section{Specimens examined and repositories}

Here we mainly consider revised data from Slovenia and, exemplarily, data from Austria, Bosnia and Herzegovina, Croatia and Italy, deposited in the central Opiliones collection, Prirodoslovni Muzej Slovenije, Ljubljana, PMSL (Natural History Museum of Slovenia), and - those collected by S. Polak - in Notranjski muzej, NM (Notranjska Museum, Postojna). The PMSL central Opiliones collection comprises the following collections: IZRK (Karst Research Institute ZRC SAZU, Postojna), NIB (National Institute of Biology), JH (Jovan Hadži), PK (Peter Kozel) and TN-LSN (Tone Novak \& Ljuba Slana Novak). Each sample has an individual code number in the brackets, consisting of an acronym of the identifier and the identification number, i.e., the current identification number in the year of identification. The acronyms are as follows: JG Jürgen Gruber, JH Jovan Hadži, CK Christian Komposch, PK Peter Kozel, SL Saška Lipovšek, JM Jochen Martens, TN Tone Novak, GR Günther Raspotnig, ASc Axel Schönhofer, LSN Ljuba Slana Novak, GŽ Gregor Žnidar. Since T. Novak identified most of the samples, these numbers are without acronyms; the acronym is added only for revised samples. In samples from other collections, we have retained the original citations.

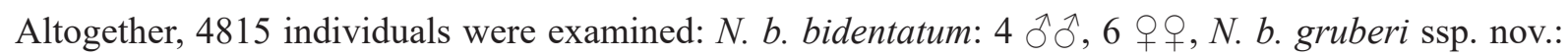

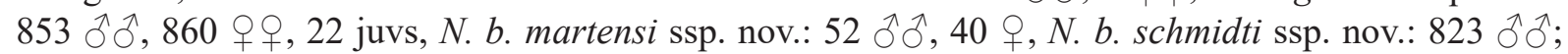

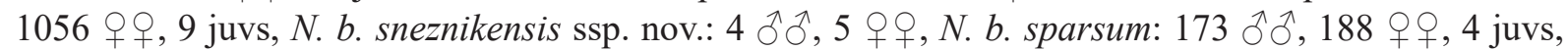

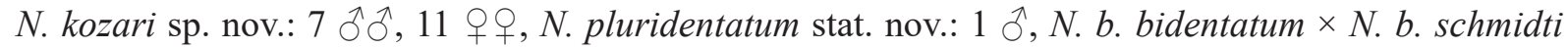

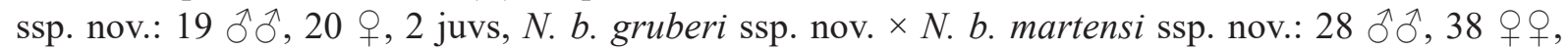
$N$. b. gruberi ssp. nov. $\times N$. b. martensi ssp. nov. $\times N$. b. schmidti ssp. nov.: 5 ô $\hat{0}, 4$ q, , N. b. gruberi ssp. nov. $\times N$. b. schmidti ssp. nov.: $93 \hat{\partial} \widehat{\partial}, 59$ 우, $N$. b. martensi ssp. nov. $\times N$. b. schmidti ssp. nov.:

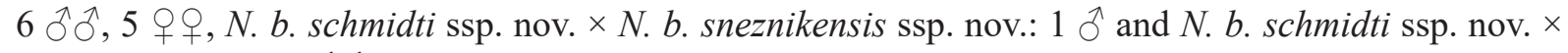
N. b. sparsum: 197 ふぇ, 198 우, 14 juvs.

\section{Repositories}

PMSL = Prirodoslovni Muzej Slovenije, Ljubljana

$\mathrm{NM} \quad=$ Notranjski muzej, Postojna

NHMW $=$ Naturhistorisches Museum Wien, Vienna 


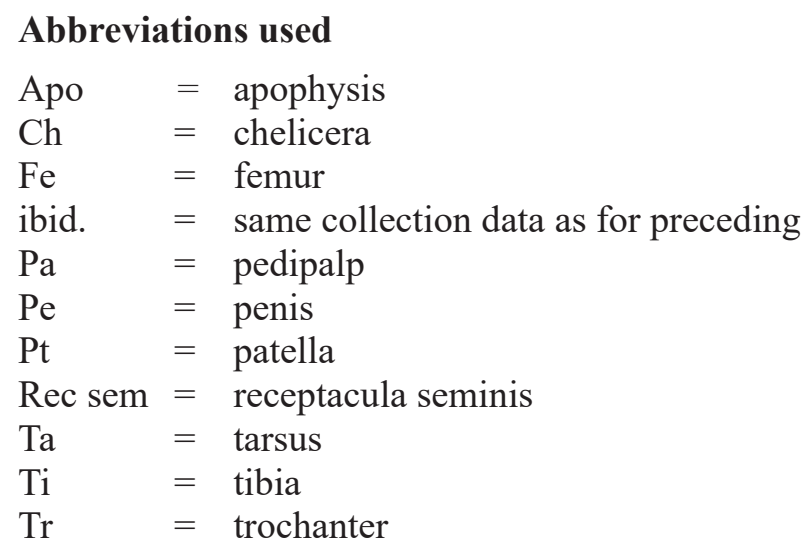

Leg numbering: I-IV.

\section{Sampling area in Slovenia}

Prior to this investigation, data on the $N . b$. complex in Slovenia were too scattered to allow proper analysis of variation within the $N . b$. complex. For the purpose of this study, we sampled $N$. bidentatum following the $10 \times 10 \mathrm{~km}$ UTM grid as the base. In this way, we provided samples in 215 UTM squares (82.4\%) covering Slovenia. In the other UTM squares, $N$. bidentatum have not been found. In locality quotations, the UTM codes serve as surrogates for GeoCoordinates. Due to the density of sampling, the UTM codes replace the GeoCoordinates of all localities within the squares. Data for the other countries were presented only exemplarily.

\section{Morphology}

The morphology of genitalia, $\mathrm{Ch}, \mathrm{Pa}$ and the presence of the saw on Fe IV were considered in taxa identification. We followed Gruber's and Martens' (1968) revision of $N$. bidentatum as the basic taxonomic reference. We included all hitherto known Nemastoma bidentatum s. lat. in the analysis, i.e., taxa having the Pe with a posteriorly indented, muscular basis, a thin, dorso-ventrally depressed truncus and a triangular glans with two pairs of articulated, lancet-like lateral spines, and a saw-like postero-lateral range of denticles on Fe IV (Fig. 1). In our study, we considered the measurements and shape of the basal Ch article and Ch-Apo (present only in males), the measurements and shape of the Pa, especially the Pa-Fe, including the number and distribution of spines, thorns, denticles, tubercles and setae, as well as characters of the glans and Rec sem. A spine is a long, rigid, acute prominence, a thorn a stout prominence with obtuse to nearly missing terminal point, a denticle is a tooth-like, mostly pointed microstructure $(<10 \mu \mathrm{m}$ high), a tubercle is a blunt mictrostructure, and a seta is a hair-like projection of cuticle (terminology according to Gruber \& Martens 1968; Martens 1978; Murphree 1988; Schulz \& Pinto-da-Roch 2007; Willemart et al. 2009, except the thorn, which is our own interpretation). The secretion field is a group of thin openings of the glandular organs on the Ch-Apo, secreting the nuptial gift that roughly coincides with a brush-like field of setae (Gruber \& Martens 1968; Martens 1978; Gruber 2007). Ch and Pa were studied in the lateral position, and the $\mathrm{Pa}$ armament was additionally examined in a medio-superior view, where this information was of supplementary importance for the presentation. The Pa-Fe minimum width/maximum width is routinely cited for the taxa. The Pa-Fe maximum width is the largest diameter, subterminally or terminally on the $\mathrm{Pa}-\mathrm{Fe}$, and the $\mathrm{Pa}-\mathrm{Fe}$ minimum width is the narrowest diameter of the $\mathrm{Pa}-\mathrm{Fe}$ at the very proximal portion or a little bit distant from the Tr-Fe joint. In some species, a terminal bonce, i.e., a head-like enlargement, is present. For descriptions, 3-5 females were taken from singletaxon populations, considering their $\mathrm{Ch}, \mathrm{Pa}$ and $\mathrm{Rec}$ sem. Typical male hybrids, i.e., those showing some specific traits beside intermediate traits not present in any subspecies, were briefly described. Since the female hybrids show only intermediate traits, we have not described their morphology separately.

We studied the morphology under a Nikon Eclipse i50 compound microscope (Nikon, Japan), with a mounted digital DS-Fi3 microscope camera, and processed with Nikon NIS-Elements D ver. 5.30.00 
software. Transmission light microscopy was combined with illumination from above. Images were manipulated for optimal quality using various applications in GIMP ver. 2.10.22. Drawings were made with a drawing tube mounted on an Olympus $\mathrm{CH} 30$ microscope, and then post-processed in the InkScape ver. 0.91 program. We combined glycerol and dry preparations in the procedures. Ovipositors were heated (up to ca $70^{\circ} \mathrm{C}$ ) in lactic acid until the ovipositor became translucent enough to examine the Rec sem. Measurements are in millimeters, if not otherwise indicated.

\title{
Ecology
}

All the taxa within the $N$. $b$. complex are specialized for living in a loose, humus organic soil horizon and litter. Specific differences in environmental requirements of different taxa deserve a separate study. Here we have roughly assessed the requirements through identification of typical phytocenosis at particular sites. Field notes on the habitat and microhabitat characteristics inhabited by particular taxon of $N$. $b$. complex were recorded to provide further ecological data. Data on ecology and habitat provide further information that may be of supporting importance in identification in particular cases.

\section{Results}

\author{
Class Arachnida Lamarck, 1801 \\ Order Opiliones Sundevall, 1833 \\ Suborder Dyspnoi Hansen \& Sørensen, 1904 \\ Family Nemastomatidae Simon, 1872 \\ Subfamily Nemastomatinae Simon, 1872
}

Genus Nemastoma C.L. Koch, 1836

Diagnosis (according to Gruber \& Martens 1968, slightly modified)

Genus of Nemastomatidae Simon, 1872, with body length 1.5-2.5 mm, with scutum compositum; uniformly black or with silver or golden lateral spots. Pe with base perpendicular to dorso-ventrally flattened truncus, glans bilaterally symmetric or subsymmetric, without or with 1-8 pairs of lateral spines or denticles.

Nemastoma bidentatum Roewer, 1914 complex

\section{Diagnosis}

Uniformly black subspecies of Nemastoma bidentatum and closely related species, with glans with two pairs of lateral lancet-like, articulated spines, and saw-like series of denticles on Fe IV.

\section{Morphology}

The Pe is characterized by a bulbous, muscular, posteriorly medially split basis, a thin truncus and triangular glans, eventually with a shoulder-like truncated tip, either slightly narrower, evenly wide or slightly wider than the truncus, with two pairs of lateral lancet-like, articulated spines. The distal part of the glans is characteristic of some taxa. The shape and size of the Ch basal article and especially of ChApo, and the shape, size and armament of $\mathrm{Pa}-\mathrm{Fe}$, and in some taxa $\mathrm{Pa}-\mathrm{Ti}$, are further diagnostic characters. Other characters, such as the Pa and leg lengths, may support identification, but are not definitively distinctive. The identification key includes both sexes in the nine pure taxa known to date, except the unknown female of $N$. pluridentatum stat. nov., and typical male hybrids.

\section{Remarks}

To date, four additional taxa of the $N$. bidentatum complex have been discovered in Slovenia: $N$. $b$. gruberi Novak, Slana Novak, Kozel \& Raspotnig ssp. nov., N. b. martensi Novak, Slana Novak \& Raspotnig ssp. nov., N. b. schmidti Novak, Raspotnig \& Slana Novak ssp. nov. and N. b. sneznikensis Novak, 
Komposch, Slana Novak \& Raspotnig ssp. nov., and one in Bosnia and Herzegovina: N. kozari Novak, Kozel, Podlesnik \& Raspotnig sp. nov. Within these new taxa, the relatively consistent external morphology of the male Ch-Apo, Pa and glans allows reliable assignment of most individuals. However, N. b. gruberi ssp. nov. shows considerable variation in morphology, especially in the $\mathrm{Pa}-\mathrm{Fe}$ armament, which in some particular cases still raises doubts about the reliability of the assignment (see the description of the taxon for more details). This is because we are still missing a broad review of potentially syntopic hybrids that should be based on data sets independent from morphology, e.g., chemical characters from highly specific scent gland secretions. Such individuals arise from some marginal localities of the area, e.g., from the very south-west of Slovenia, where we still have not been able to exclude the eventual existence of hybrids with $N$. b. schmidti ssp. nov. Morphological differences between females based on the Ch, Pa and Rec sem characters are less conspicuous, but allow correct identification of over $90 \%$ of individuals belonging to pure subspecies. Besides, a pairwise vicariance in most neighboring subspecies reveals their different ecological requirements, resulting in niche partitioning. On the other hand, hybrids in the contact zones of adjacent populations of some taxa reveal active gene exchange, supporting the subspecies status of these taxa. Nemastoma bidentatum in Austria deserves a separate treatise, while scarce records from the Balkan countries do not allow any further conclusion for the time being.

Thus, to date, there are four species in the Nemastoma b. complex in total: Nemastoma bidentatum Roewer, 1914, N. relictum Gruber \& Martens, 1968 stat. nov. and N. pluridentatum (Hadži, 1973) stat. nov., both raised to the species level, and the newly discovered N. kozari Novak, Kozel, Podlesnik \& Raspotnig sp. nov.

Nemastoma bidentatum Roewer, 1914

Nemastoma bidentatum Roewer, 1914: 141, figs 20a-d (original description) [partim; type series also includes $N$. triste].

Nemastoma bidentatum - Roewer 1923: 658, figs 817a-d [partim; type series also includes N. triste]. — Gruber \& Martens 1968: 141-142 (redescription). — Martens 1978: 106-107. — Schönhofer 2013: 36 .

Diagnosis (according to Gruber \& Martens 1968, slightly modified)

Species of the Nemastoma $b$. complex with male Pa-Fe having either at least two conspicuous spines or long, rigid setae instead of some spines, or 6-7 irregular tubercles, or 5-11 denticles, and Pa-Fe min:max width 1:2.5-3.5, Rec sem with 1 or none tubular and 12-18 balloon-like vesicles.

\section{Remarks}

To date, the species includes six subspecies: $N$. b. bidentatum Roewer, 1914, $N$. b. sparsum Gruber \& Martens, 1968, and the newly discovered N. b. gruberi Novak, Slana Novak, Kozel \& Raspotnig ssp. nov., N. b. martensi Novak, Slana Novak \& Raspotnig ssp. nov., N. b. schmidti Novak, Raspotnig \& Slana Novak ssp. nov. and N. b. sneznikensis Novak, Komposch, Slana Novak \& Raspotnig ssp. nov.

Nemastoma bidentatum bidentatum Roewer, 1914

Figs 2-3, 4A, 5A, 6A, 7B, 8B, 9A, 10A, 11B, 12A, 13A; Tables 1-2

Nemastoma bidentatum Roewer, 1914: 141, figs 20a-d (original description) [partim; type series also includes $N$. triste].

Nemastoma bidentatum - Roewer 1923: 658, figs 817a-d [partim; type series also includes N. triste]; 1951: 132 [partim; the description also refers to $N$. bidentatum sparsum]. 
Nemastoma bidentatum bidentatum - Gruber \& Martens 1968: 143-146, figs 4-6, 23 (redescription) [partim; type series also includes N. bidentatum schmidti ssp. nov.]. — Martens 1978: 107-111, figs 140-141 [partim; type series also includes $N$. bidentatum schmidti ssp. nov.]. - Komposch \& Gruber 2004: 485 [partim; type series also includes N. bidentatum schmidti ssp. nov.]. — Novak et al. 2006: 269. — Schönhofer 2013: 36.

Diagnosis (according to Gruber \& Martens 1968, slightly modified)

Subspecies of $N$. bidentatum with large, arched dorsal hump on male Ch basal article, and large, apically, sagittally shallowly bifid, quarter moon-like Ch-Apo, $\sim 2.4$ times as high as wide. Pa-Fe distinctively club-shaped (Pa-Fe min:max width 1:3.5), with 3 stout spines in distal Pa-Fe quarter, spine 2 the largest and longest. Pa-Pt Apo equilateral triangular with rounded apex. Pa-Ti with conspicuous proximal dorsomedial hump. Glans isosceles triangular, terminally rounded-truncated. Rec sem of 1 tubular and 12 elongated balloon-like vesicles.

\section{Material examined}

AUSTRIA - VM44 • 1 q; Bärental; 23 Apr. 2018; L. Slana Novak and T. Novak leg. (6/2018); PMSL.

SLOVENIA - VM44 • 2 우; Ljubelj; 1 Jun. 1993; S. Brelih leg. (799/1998, rev. 2018); PMSL・ 1 ô, 1 †; Ljubeljski prelaz; 20 Jun. 2018; T. Novak leg. (23/2018); PMSL・ 3 §ิ ô, 2 우; ibid.; 23 Jul. 2021; L. Slana Novak and T. Novak leg. (298/2021); PMSL.

Description (according to Gruber \& Martens 1968, slightly modified)

Male (from Ljubelj)

BoDy. Length 1.5-1.7.

Chelicerae. Ch basal article with large, arched hump in front of dorsal indentation, and large ventral triangular hump; Ch-Apo conspicuously quarter moon-like, Apo apex sagittally bilobed, medial lobe with inconspicuous apical pinnacle (Figs 4A, 6A, 9A). Secretion field apically medially. Proximal article length 0.57 , distal article length 0.54 , max width 0.17 , movable finger length 0.25 .

PediPalPs. Pa-Tr with high, subequilateral dorsal hump. Pa-Fe distinctively club-shaped (Pa-Fe min:max width $\sim 1: 3.5$ ), ventrally bent; with 3, rarely 4 stout spines in distal Pa-Fe quarter; spine 2 the largest, with 2-3 pinnacles. Pa-Pt distinctively ventrally bent, with large, stout, apically rounded medio-ventral Pt-Apo. Pa-Ti straight or slightly bent dorsally, with small, but distinct proximal dorso-medial hump. Pa-Ta terminally narrowing, conically oval (Figs 5A, 6A, 10A). Pa article lengths in Table 1.

Penis. Pe length 1.7, Pe basis $1 / 3$ Pe length, glans isosceles triangular, terminally rounded-truncated (Figs 7B, 8B).

LeGs. Leg article lengths in Tables 1-2.

Female (from Ljubelj)

BoDy. Length 1.6-1.8.

Chelicerae. Ch with subparallel dorsal and ventral margins, slightly arched dorsally, Ch length:width $\sim 2.6: 1$ (Fig. 13A). Proximal article length 0.57, distal article length 0.63, max width 0.21, movable finger length 0.32 . 
Table 1. Length of appendage-segments in millimeters in N. bidentatum bidentatum Roewer, 1914 from Ljubelj, Slovenia: $\widehat{\partial}(+)$.

\begin{tabular}{lccccccc}
\hline & Trochanter & Femur & Patella & Tibia & Metatarsus & Tarsus & Total length \\
\hline Pedipalp & $0.25(0.33)$ & $0.76(0.80)$ & $0.53(0.56)$ & $0.46(0.49)$ & - & $0.29(0.31)$ & $2.29(2.49)$ \\
Leg I & $0.23(0.24)$ & $0.68(0.70)$ & $0.39(0.38)$ & $0.61(0.64)$ & $1.06(1.05)$ & $0.97(0.98)$ & $3.94(3.99)$ \\
Leg II & $0.27(0.31)$ & $1.30(1.56)$ & $0.47(0.45)$ & $1.13(1.01)$ & $2.03(1.68)$ & $0.84(1.47)$ & $5.98(6.48)$ \\
Leg III & $0.19(0.23)$ & $0.87(0.96)$ & $0.36(0.35)$ & $0.61(0.64)$ & $1.15(1.17)$ & $0.88(0.91)$ & $4.06(4.26)$ \\
Leg IV & $0.25(0.25)$ & $1.32(1.21)$ & $0.39(0.44)$ & $0.78(0.89)$ & $1.84(1.84)$ & $1.18(1.16)$ & $5.75(5.79)$ \\
\hline
\end{tabular}

Table 2. Length of appendage-segments in millimeters (trochanters not presented) in $N$. bidentatum bidentatum Roewer, 1914 from Bärental, Austria: $\widehat{\partial}$ ( $(+)$ (from Gruber \& Martens 1968).

\begin{tabular}{lcccccc}
\hline & Femur & Patella & Tibia & Metatarsus & Tarsus & Total length \\
\hline Leg I & $0.75(0.75)$ & $0.35(0.35)$ & $0.55(0.60)$ & $1.00(0.90)$ & $0.85(0.85)$ & $3.50(3.50)$ \\
Leg II & $1.20(0.80)$ & $0.35(0.40)$ & $1.05(1.05)$ & $1.90(1.95)$ & $1.55(1.60)$ & $6.05(5.80)$ \\
Leg III & $0.75(0.85)$ & $0.30(0.35)$ & $0.60(0.55)$ & $1.15(1.05)$ & $0.80(0.80)$ & $3.60(3.60)$ \\
Leg IV & $1.25(1.30)$ & $0.35(0.35)$ & $0.75(0.75)$ & $1.75(1.70)$ & $1.05(1.05)$ & $5.15(5.15)$ \\
\hline
\end{tabular}

PediPALPS. Pa-Tr long, Pa-Tr length:width 2.5:1, Pa-Tr dorsal margin with low hump with long, straight posterior portion and stair-like front indentation in distal quarter; Pa-Fe max:min width $~ 1.9: 1$; Pa-Ti straight, simply rod-shaped; Pa-Ta narrow long-oval (Fig. 13A). Pa article lengths in Table 1.

OviPositor. Ovipositor length 1.05, Rec sem with one tubular and 12 elongated balloon-like vesicles (Fig. 11B).

LEGs. Leg article lengths in Tables 1-2.

\section{Remarks}

The type locality Comana Vlasca in Romania (Roewer 1914) is erroneous, since besides $N$. b. sparsum (sic! not described by Roewer 1914), the type series also contains $N$. b. bidentatum and $N$. triste, both endemic to the Eastern Alps (Gruber \& Martens 1968: 143). Later, Roewer (1951) designated a type specimen from the type series from an unknown locality, but the mixed composition of the type series remained unresolved (Gruber \& Martens 1968). Thus, there was a need to clarify the type locality (ICZN 1999: Art. 75.3.1.). Respecting both Roewer's drawing of the male Ch characteristic of individuals in the Karawanken/Karavane Mts and the syntopy of the Roewer's type specimens with N. triste, Gruber \& Martens (1968) redescribed the nominal subspecies N. b. bidentatum upon a neotype (Gruber \& Martens 1968: figs 4-6) from Bärental, Carinthia, Austria (not mapped in Gruber \& Martens 1968: fig. 24). Bärental is considered the type locality of the type subspecies. However, according to our findings the figured Pe tip from Leutschach/Lučine, Styria, Austria (Gruber \& Martens 1968: fig. 3), considered under the type subspecies, belonged to $N$. b. schmidti ssp. nov. All so far published evidence on N. b. bidentatum in Slovenia, Croatia and NE Italy (Hadži 1927, 1928; Gruber \& Martens 1968; Martens 1978; Novak \& Gruber 2000; Komposch \& Gruber 2004; Novak 2004a, 2004b, 2005b, 2005c; Novak et al. 1984, 1995, $1996,2002,2006,2017)$ refer to other taxa. The type series deposited in Naturhistorisches Museum Wien (Gruber \& Martens 1968) also includes N. b. schmidti ssp. nov. See remarks under N. pluridentatum stat. nov., $N$. b. schmidti ssp. nov., $N$. b. gruberi ssp. nov. $\times N$. b. schmidti ssp. nov. and $N . b$. martensi ssp. nov. $\times N$. b. schmidti ssp. nov. 


\section{Distribution}

The South-Eastern Alps: Austria, Slovenia (Gruber \& Martens 1968; Martens 1978), perhaps endemic to the Karawanken/Karavanke Mts. Vertical distribution (incomplete data): 980-1407 m a.s.l. (partly Gruber \& Martens 1968 and Martens 1978; this work). Type locality: Bärental/Zavrh-Rute (46.47 N, $14.15^{\circ}$ E, 980 m a.s.1.) near Feistritz im Rosental/Bistrica v Rožu, Carinthia, Austria.

\section{Ecology}

Montane subspecies of $N$. bidentatum inhabiting submontane and montane neutrophile fir forests within the area of distribution of the montane beech forests, dominated by Abies alba Mill., with a great admixture of Picea abies (L.) H.Karst. and Fagus sylvatica L. Phenology: adults probably eurychronous.

Nemastoma bidentatum sparsum Gruber \& Martens, 1968 Figs 2-3, 4B, 5B, 6B, 7C, 8C, 9B, 10B, 11A, C, 12B, 13B; Tables 3-4

Nemastoma bidentatum sparsum Gruber \& Martens, 1968: 146-147, figs 1-2, 7-11 (original description) [partim; the type series also includes $N$. bidentatum gruberi ssp. nov.].

Nemastoma bidentatum - Roewer 1914: 141 [partim: type series also includes N. bidentatum bidentatum and N. triste]. — Šilhavý 1956: 131, figs 243-246. — Loksa 1962: 267, figs 3a-f. — Gruber \& Martens 1968: 146-147, figs 1-2, 7-11. - Staręga 1976: 313-315, figs 25-27. — Martens 1978: 111-112, figs 143-145, 147, 150; 2006: 182-184, figs 20, 21c-d, 22a-h. - Novak \& Gruber 2000: 286. - Novak et al. 2002: 136 [partim: Radenci]; 2006: 269. — Komposch \& Gruber 2004: 485.

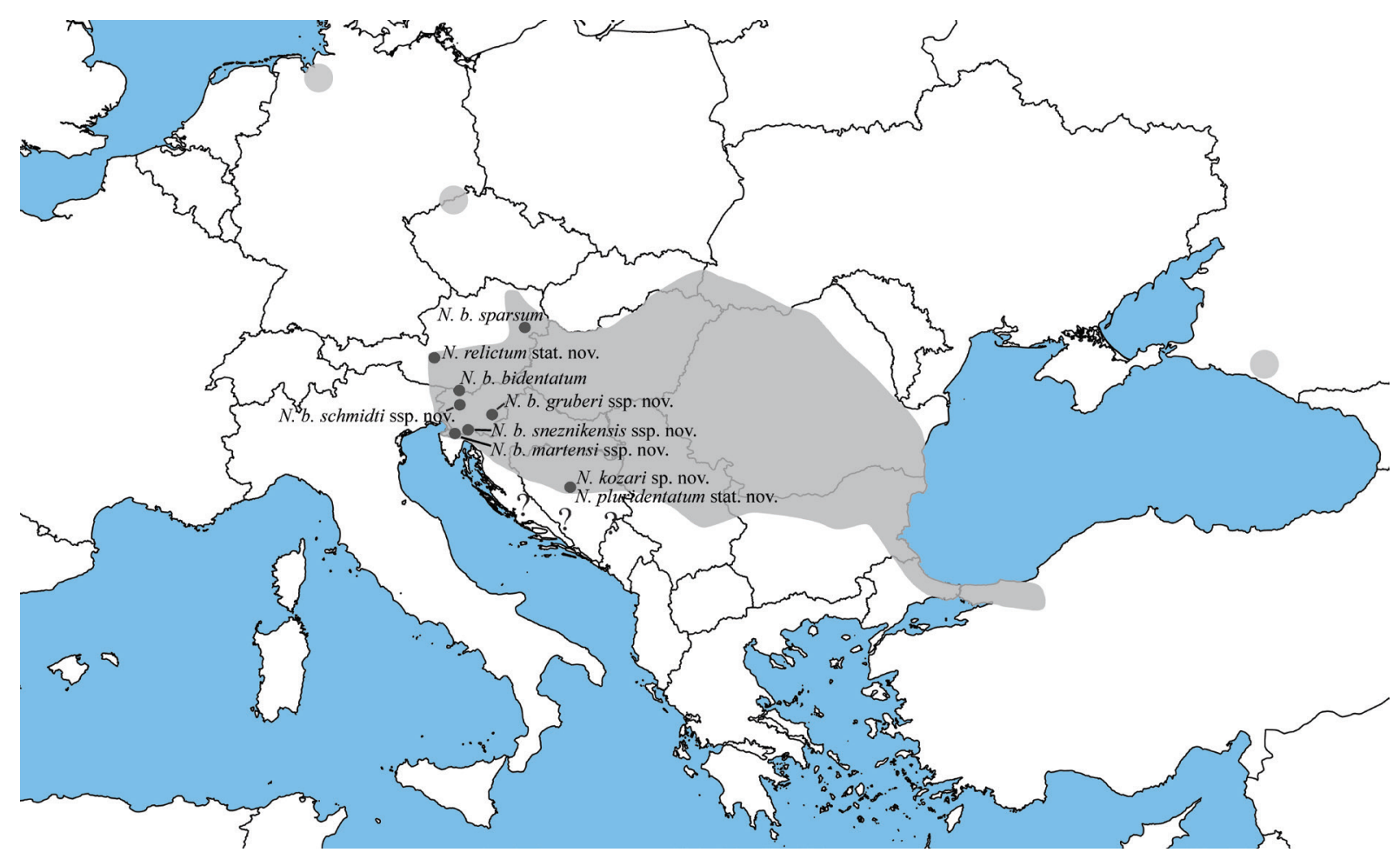

Fig. 2. The complete distributional area of the Nemastoma bidentatum Roewer, 1914 complex, according to Gruber \& Martens (1968), Martens (2006), Schönhofer \& Holle (2007) and own data, with marked positions of the type localities of the taxa. 
— Novak 2004b: 245 [partim: Breznica, Slavonski brod, Spačva near Županja]; $2005 \mathrm{a}: 315$ [partim: Bosanski Brod]. — Schönhofer \& Holle 2007: 25, figs 2a-i. — Schönhofer 2013: 36.

Diagnosis (according to Gruber \& Martens 1968, slightly modified)

Male Ch basal article with nearly straight dorsal margin in front of dorsal indentation, and short, roundly oblong Ch-Apo, with small apical pinnacle. Pa-Fe moderately club-shaped (Pa-Fe min:max width 1:2.5), with 2 distal, pointed spines, spine 2 the largest. Pa-Pt Apo conical. Glans equilaterally triangular. Rec sem with 14 balloon-like vesicles.

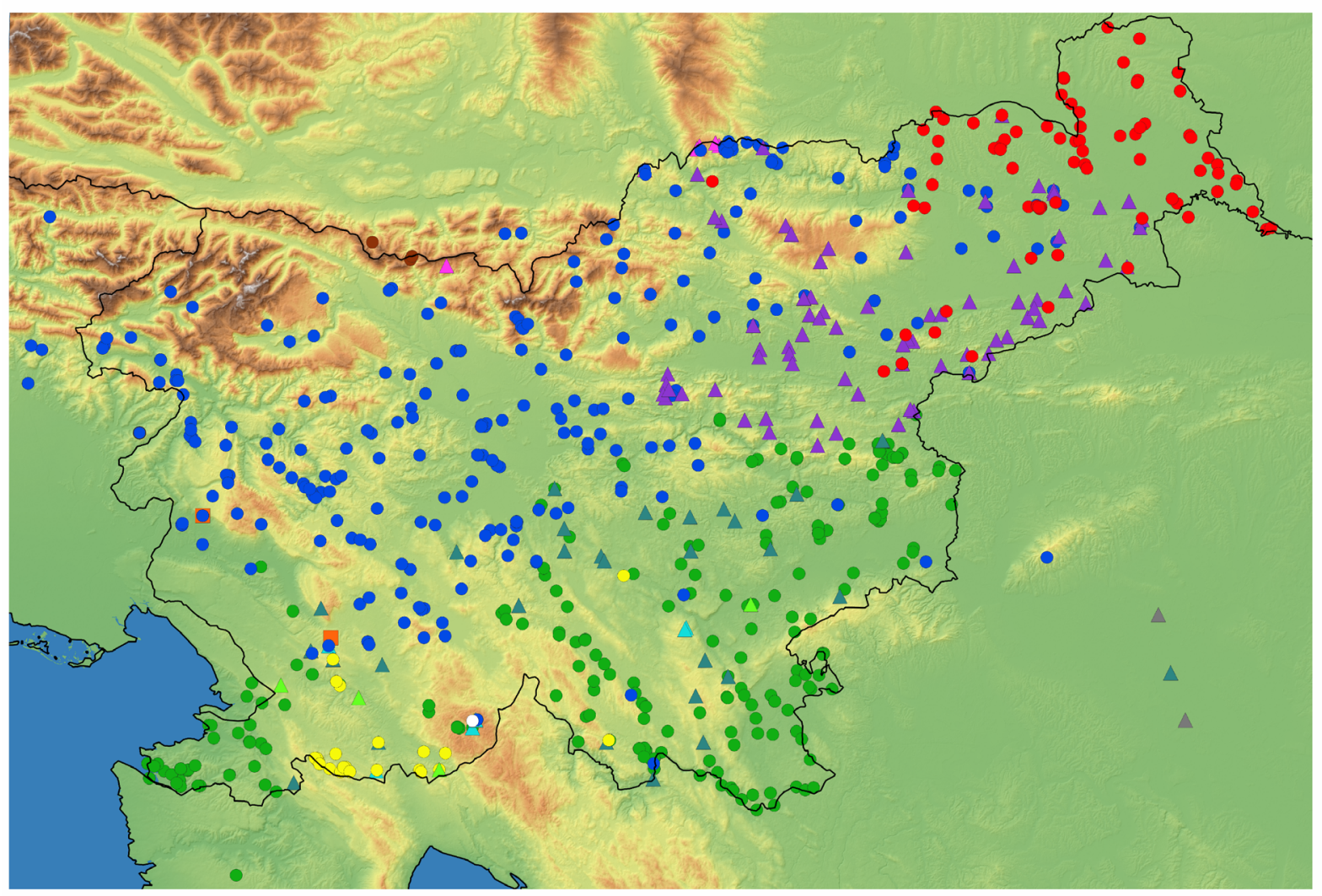

- N. b. bidentatum

- N. b. sparsum

- N. b. gruberi ssp. nov.

o N. b. martensi ssp. nov.

- N. b. schmidti ssp. nov.

o $N$. b. sneznikensis ssp. nov.

$\triangle N$. b. bidentatum $\times$ N. b. schmidti ssp. nov.

$\triangle N$. b. gruberi ssp. nov. x N. b. martensi ssp. nov.

$\square$ N. b. gruberi ssp. nov. x N. b. martensi ssp. nov. x N. b. schmidti ssp. nov.

$\Delta N$. b. gruberi ssp. nov. x N. b. schmidti ssp. nov.

$\triangle N$. b. gruberi ssp. nov. x $N$. b. sparsum ssp. nov.

$\triangle N . b$. martensi ssp. nov. x N. b. schmidti ssp. nov.

$\Delta N$. b. schmidti ssp. nov. x N. b. sparsum ssp. nov.

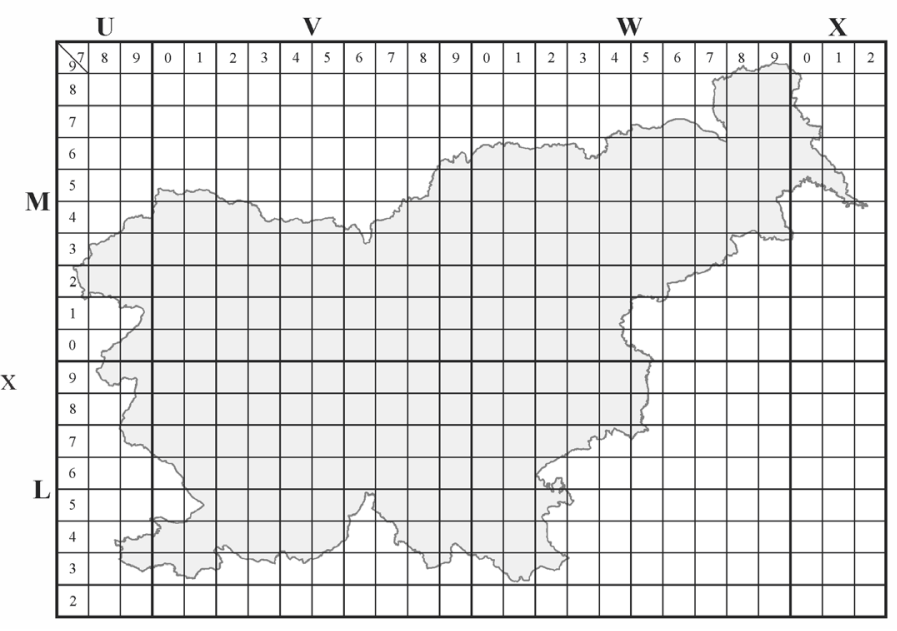

Fig. 3. Distributional areas of six subspecies (circles) and hybrids (triangles, squares) within the Nemastoma bidentatum Roewer, 1914 in Slovenia and adjacent regions. Note that many localities overlap on the map. 
NOVAK T. et al., Nemastoma bidentatum (Opiliones: Nemastomatidae) revison

\section{Material examined}

CROATIA - XM05 • 1 q; Jurovčak; 3 Apr. 1983; T. Novak and L. Slana Novak leg. (LSN 7/1983, TN rev. 2008); PMSL.

SLOVENIA - WM06 • 1 đ’; Cvitrško sedlo, Otiški vrh; 28 May 2008; T. Novak leg. (160/2011); PMSL. -WM42 - 1 đ, 2 우, 1 juv.; Mestinje; 4 Aug. 1983; T. Novak, M. Štangelj and L. Slana Novak leg. (LSN 43/1983); PMSL・ 1 ô, 1 q; ibid.; 13 Jul. 2002; L. Slana Novak and T. Novak leg. (1138/2002); PMSL • 1 q; Šmarje pri Jelšah; 6 Apr. 1986 (1169/1997, rev. 2015); PMSL • 1 q; Zgornje Poljčane; 13 Jul. 2002; L. Slana Novak and T. Novak leg. (1160/2002); PMSL. - WM45 • 1 ð̊; Ledine; 25 Jul. 1987; F. Janžekovič leg. (7/1999); PMSL • 1 đ̃; Maribor; 24 Aug. 1986; Malavašić and Koren leg. (265/2002); PMSL. - WM47 • 1 क ; Kresnica; 20 Apr. 2008; L. Slana Novak and T. Novak leg. (162/2011); PMSL. - WM52 - 1 đે; Maršečka vas; 11 Oct. 1997; L. Slana Novak and T. Novak leg. (1893/1998);

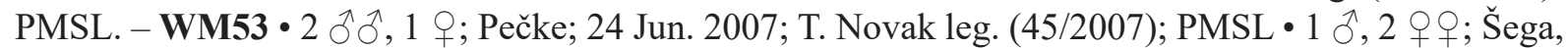
Makole; 9 Apr. 1983; T. Novak, M. Štangelj and L. Slana Novak leg. (LSN 70/1983); PMSL. - WM56 - 1 9; Kaniža; 20 May 1983; T. Novak, M. Štangelj and L. Slana Novak leg. (LSN 80/1983); PMSL • 1 ㅇ; Mt. Piramida, Maribor, 1-30 Nov. 1978; (909/1997, rev. 2015); PMSL・1 9 ; Spodnje Dobrenje; 23 Apr. 2008; L. Slana Novak and T. Novak leg. (165/2011); PMSL・Ranca, Pesnica. - WM57 • 1 万’; Ceršak; 20 Apr. 2008; L. Slana Novak and T. Novak leg. (113/2011); PMSL・ 1 đ; Kozjak pri Ceršaku; 20 Apr. 2008; L. Slana Novak and T. Novak leg. (116/2011); PMSL. - WM66 • 2 q 9 ; Črnetina; 23 Apr. 2008; L. Slana Novak and T. Novak leg. (163/2011); PMSL • 1 đ̂; Kremberg; 4 Jun. 1984; T. Novak, M. Slana Novak and L. Slana Novak leg. (LSN 343/1985); PMSL・ 2 ổ; ibid.; 23 Apr. 2008; L. Slana Novak and T. Novak leg. (114/2011); PMSL • 1 9; Spodnji Žerjavci; 23 Apr. 2008; L. Slana Novak and T. Novak leg. (115/2011); PMSL. - WM67 • 1 đ̊; Sv. Ana; 3 Jul. 2002; T. Novak leg. (799/2002); PMSL - 1 o; Nasova; 3 Jun. 1984; L. Slana Novak and T. Novak leg. (314/2002, rev. 2015); PMSL • 1 o; Stogovci; 13 Apr. 1984; T. Novak, M. Slana Novak and L. Slana Novak leg. (LSN 323/1985); PMSL • 1 ô, 1 क; Zgornja Velka; 20 Mar. 1983; T. Novak, M. Štangelj and L. Slana Novak leg. (LSN 8/1983); PMSL. - WM73 • 1 क ; Repišče; 24 Jun. 2007; T. Novak leg. (31/2007, rev. 2015); PMSL. - WM74 •

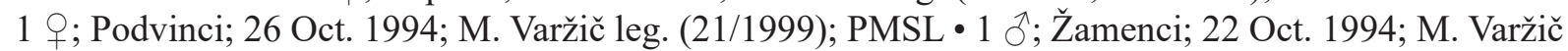
leg. (18/1999); PMSL・ 1 ơ; ibid.; (20/1999); PMSL・ 1 đ̊; ibid.; 23 Oct. 1994; M. Varžič leg. (19/1999, rev. 2015); PMSL. - WM75 • 1 o; Trnovce; 2 Jun. 2002; L. Slana Novak and T. Novak leg. (760/2002);

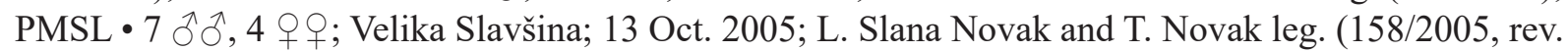

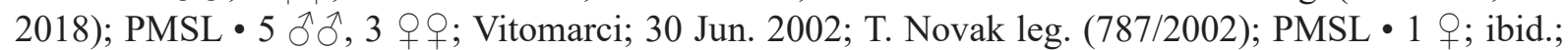
3 Jul. 2002; T. Novak leg. (792/2002); PMSL・ 1 ö; ibid.; (797/2002); PMSL・ 1 क; ibid.; 3 Jul. 2002; T. Novak leg. (794/2002, rev. 2008); PMSL・ 1 क; ibid.; (796/2002); PMSL・ 1 O;; ibid.; (797/2002); PMSL・ 1 ; ; ibid.; (800/2002); PMSL・ 1 क; ibid.; 2 Oct. 2002; T. Novak leg. (1280/2002); PMSL・ 1 \%; ibid.; (1279/2002); PMSL. - WM77 • 1 q; Črešnjevci; 24 Apr. 1983; T. Novak, M. Štangelj and L. Slana Novak leg. (LSN 79/1983, TN rev. 2008); PMSL • 1 o, 1 q; Korovci; 7 Apr. 1984; L. Slana Novak and T. Novak leg. (LSN 191/1985); PMSL・ 1 Ō, 1 q; Lomanoše; 1 Oct. 1983; T. Novak, M. Slana Novak and L. Slana Novak leg. (LSN 110/1983); PMSL. - WM78 • 1 ک ; Gerlinci; 11 May 1986; T. Novak and L. Slana Novak leg. (LSN 139/1986); PMSL. - WM86 • 2 ふ઼

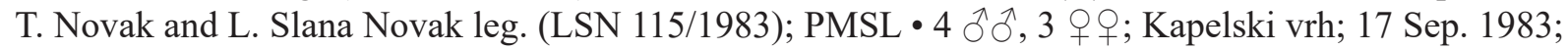
T. Novak, M. Slana Novak and L. Slana Novak leg. (LSN 112/1983); PMSL • 1 đ̊; Murski vrh; 19 May 1984; L. Slana Novak and T. Novak leg. (LSN 240/1984); PMSL • 1 + ; Radenci; 25 Jul. 1983; M. Slana

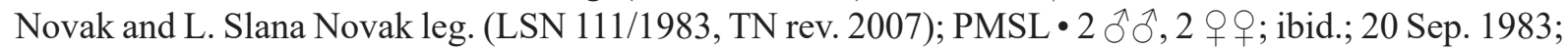
L. Slana Novak leg. (LSN 119/1983); PMSL・ 1 đ̌; Rihtarovci; 26 Feb. 1997; B. Drovenik leg. (94/2001); PMSL. - WM87 • 3 ふึત, 3 우; Cankova; 15 Sep. 1983; T. Novak and L. Slana Novak leg. (LSN 114/1983); PMSL • 3 ổ 3 , 3 우; Skakovci; 27 Mar. 1983; T. Novak and L. Slana Novak leg. (LSN 4/1983); PMSL • 1 đ̊; Sodišinci, Radenci; 19 Aug. 1983; T. Novak and L. Slana Novak leg. (LSN 68/1983); PMSL. - WM88 • 1 đ̊; Gerlinci; 11 Aug. 1983; L. Slana Novak, M. Slana Novak and T. Novak leg. (1322/1998); PMSL. - WM89 • 2 ふ઼ ${ }^{2} 4$ 우, 1 juv.; Trdkova; 30 Jul. 1983; T. Novak, M. Slana 
Novak and L. Slana Novak leg. (LSN 49/1983); PMSL. - WM94 • 1 क; Pavlovci; 15 Apr. 2000; L. Slana Novak and T. Novak leg. (744/2002); PMSL. - WM95 • 1 क ; Podgradje, Ljutomer; 27 May 2001; S. Brelih leg. (125/2001); PMSL. - WM96 - 1 juv.; Bratonci; 9 Aug. 1983; T. Novak, M. Slana Novak and L. Slana Novak leg. (LSN 335/1985, TN rev. 2008); PMSL. - WM97 • 1 ð̊; Andrejci; 28 Jul. 1981; T. Novak leg. (1188/1981, JG det.); PMSL・ 1 đ; Moravci; 16 Mar. 1984; T. Novak and L. Slana Novak leg. (LSN 243/1984); PMSL • 4 ठో 3 웅 Rakičan - Noršinci; 23 Sep. 1984; L. Slana Novak leg. (322/1985); PMSL・ 1 ô, 2 우우 ibid.;

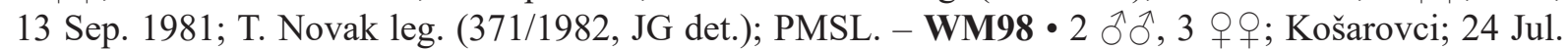
1982; L. Slana Novak, T. Novak and M. Slana Novak leg. (529/1982, JG det.); PMSL • 1 \&; ibid.; 1 Sep. 1984; L. Slana Novak, M. Slana Novak and T. Novak leg. (LSN 318/1985); PMSL・ 1 đ, 3 q o+; Mačkovci; 31 Jul. 1983; T. Novak, M. Slana Novak and L. Slana Novak leg. (LSN 67/1983); PMSL. - WM99 • 2 ô ${ }^{2}$; Lucova; 2 Jun. 1984; L. Slana Novak, M. Slana Novak and T. Novak leg. (LSN 239/1984); PMSL.

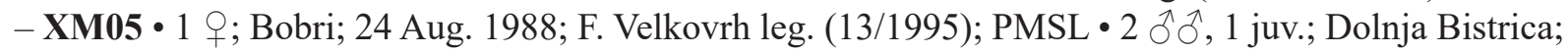

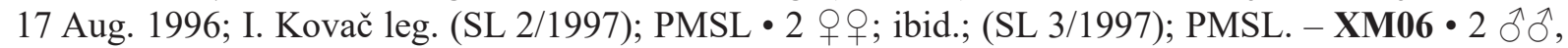

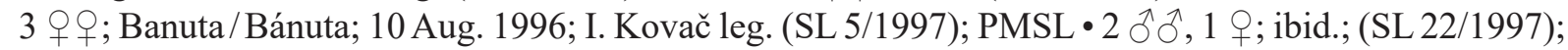

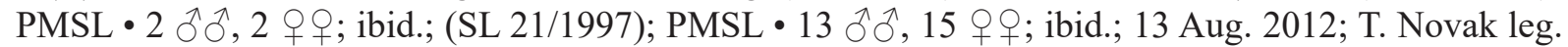
(GR 3837-3849, 3792-3797, TN det.); PMSL・ 4 ๙ð, 3 우; ibid.; (GR 3770-3776); PMSL・ several specs; Polanski log, Mala Polana; 17 Jul. 2000; T. Novak leg.; PMSL・ 1 ô, 1 ९; Radmožanci/Radamos; 17 Mar. 1984; T. Novak and L. Slana Novak leg. (LSN 109/1984); PMSL. - XM07 • 4 ô ô, 3 q 우; Dobrovnik/Dobronak; 8 Aug. 1983; T. Novak, M. Slana Novak and L. Slana Novak leg. (LSN 57/1983); PMSL • 1 क, 1 juv.; ibid.; 18 Aug. 1996; I. Kovač leg. (SL 1/1997); PMSL. - XM08 • 3 ô ô, 1 o ; Prosenjakovci/Pártosfalva; 8 Aug. 1983; T. Novak, M. Slana Novak and L. Slana Novak leg. (LSN

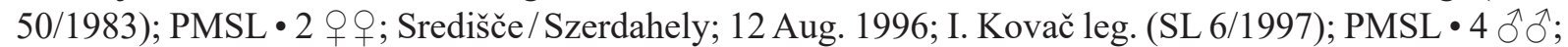
ibid.; 24 Aug. 1996; I. Kovač leg. (SL 7/1997); PMSL・ 2 우; ibid.; (SL 8/1997); PMSL・ 1 ภ , 3 o ㅇ;

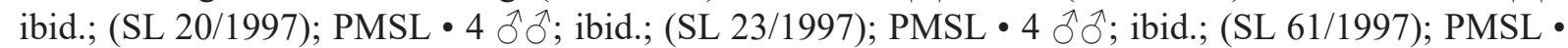

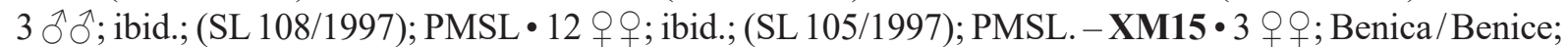

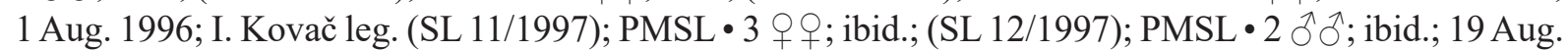
1996; I. Kovač leg. (SL 25/1997); PMSL・ 2 Q 9 ; ibid.; (SL 26/1997); PMSL・ 5 o 9 ; ibid.; (SL 28/1997);

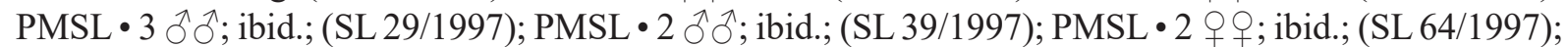
PMSL・ 1 ô, 1 O ; ibid.; (SL 65/1997); PMSL・ 1 ô, 3 우; Gaberje/Gyetyános; 22 Aug. 1996; I. Kovač leg. (SL 9/1997); PMSL・ 1 q; ibid.; (SL 10/1997); PMSL・ 1 ô; ibid.; (SL 15/1997); PMSL • 3 q ibid.; (SL 16/1997); PMSL・ 1 \&; ibid.; 12 Oct. 1996; I. Kovač leg. (SL 30/1997); PMSL・ 2 o 9 ; ibid.; (SL 55/1997); PMSL・ 5 $\widehat{\jmath}$; ibid.; 19 Oct. 1996 ( SL 17/1997); PMSL・ 4 $\widehat{\jmath}, 4$ 우 ; ibid.; (SL 24/1997); PMSL • 4 우; ibid.; (SL 103/1997); PMSL • 8 우; ibid.; (SL 104/1997); PMSL・ 2 우; ibid.;

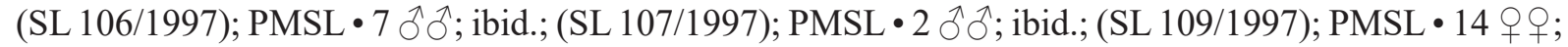
ibid.; 22 Aug. 1997; I. Kovač leg. (SL 27/1997); PMSL・ 1 ô, 1 juv.; ibid.; (SL 32/1997); PMSL・ 1 ð’;

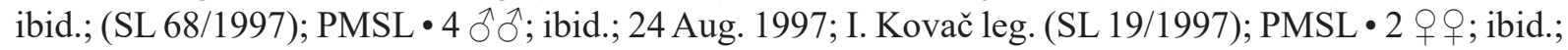
28 Aug. 1997; I. Kovač leg. (SL 13/1997); PMSL・ 2 đ̋ 1997; I. Kovač leg. (SL 4/1997); PMSL・ 4 ổ ô; ibid.; (SL 20/1997); PMSL・ 1 O; ibid.; (SL 56/1997);

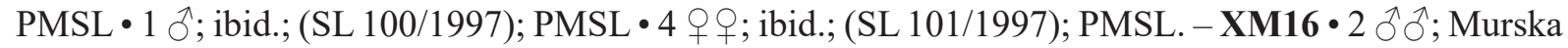
šuma; 29 Aug. 1998; T. Novak leg. (1538/1998); PMSL • several specs; ibid.; 18 Jun. 2001; T. Novak leg.; PMSL • 1 Õ, 1 q; Dolgovaške Gorice/Hossúfaluhegy; 31 Mar. 1984; T. Novak, D. Kuhar and L. Slana Novak leg. (LSN 141/1984); PMSL・ 4 ふ઼ 1 \%; Lendavske gorice/Lendvahegy; 17 Aug. 1996; I. Kovač leg. (SL 31/1997); PMSL. - XM25 • 1 đ;; Muriša, Murska šuma; B. Drovenik and A. Pirnat leg. (26/2005); PMSL.

Description (according to Gruber \& Martens 1968, slightly modified)

Male (from Prosenjakovci)

BoDy. Length 1.5-2.0. Males from Slovenia larger than those from the type locality. Length 1.96, width 1.44 
CHeLICERAE. Ch basal article with nearly straight dorsal margin in front of dorsal indentation and small ventral hump, Ch-Apo short, roundly oblong, with convex anterior margin and small apical pinnacle (Figs 4B, 6B, 9B). Secretion field medially. Proximal article length 0.53, distal article length 0.59, max width 0.18 , movable finger length 0.25 .

PEDIPALPS. Pa-Tr with highly arched dorsal hump. Pa-Fe moderately club-shaped (Pa-Fe min:max width $\sim 1: 2.5$ ), slightly ventrally bent, with 2 stout, pointed spines in distal Pa-Fe quarter; spine 2 the largest. Pa-Pt Apo triangular-conical. Pa-Ti simple, straight. Pa-Ta conically tapering (Figs 5B, 6B). Pa article lengths in Table 3.

PenIs. Pe length 1.6, Pe basis $1 / 4$ Pe length, glans equilaterally triangular (Figs 7C, 8C).

LEGS. Leg article lengths in Tables 3-4.

Female (from Prosenjakovci)

BoDy. Body length 1.6-2.4. Females from Slovenia larger than those from the type locality. Body length 2.40, body width 1.97 .

Chelicerae. Ch basal article with low-arched dorsal margin in front of dorsal indentation, and straight ventral margin with triangular hump, Ch basal article length:width $>2.6$ : 1. Proximal article length 0.57 , distal article length 0.63 , max width 0.21 , movable finger length 0.32 (Fig. 13B).

PedipalPs. Pa-Tr with moderately high, arched dorsal margin; Pa-Fe with distal bonce, Pa-Fe max:min width 1.7:1; Pa-Ti elongated-oval (13B). Pa article lengths in Table 3.

OviPositor. Ovipositor length 1.0, Rec sem with 14 balloon-like vesicles (Fig. 11A, C).

LEGS. Leg article lengths in Tables 3-4.

\section{Remarks}

In Slovenia, $N$. b. sparsum lives in the northeast, at the margins of the Pannonian basin (Martens \& Gruber 1968; Martens 1978; Kovač 1997). The type series deposited in Naturhistorisches Museum Wien (Gruber \& Martens 1968) also includes N. b. gruberi ssp. nov. distributed south of the Sava River in Slovenia and Croatia. All prior identifications of N. b. sparsum south of the Sava River (Novak et al. 1995, 2002; Novak 2005b) refer to N. b. gruberi ssp. nov. Martens (1978: 112) reported a sparsum-like population living in the mountain pine association (Hyperico grisebachii-Pinetum mugo Horvat 1938) on Mt. Veliki Snežnik (the peak at $1796 \mathrm{~m}$ ) at least up to the altitude of $1650 \mathrm{~m}$ a.s.l. These individuals may refer to $N$. b. gruberi ssp. nov., $N$. b. martensi ssp. nov. or $N$. b. sneznikensis ssp. nov. Individuals from Bosnia and Herzegovina (Mt. Plasa, Jajce) and Dalmatia, Croatia (Split) (Gruber \& Martens 1968) should be revised for identification. See remarks under $N$. b. gruberi ssp. nov. and $N$. b. gruberi ssp. nov. $\times$ N. b. schmidti ssp. nov., N. b. martensi ssp. nov. and N. b. sneznikensis ssp. nov.

\section{Distribution}

Central and eastern Europe: Austria, Bulgaria, Bosnia and Herzegovina, Czech Republic, Denmark, Hungary, Croatia, Montenegro, Romania, Russia, Slovakia, Slovenia, Serbia and Turkey (Gruber \& Martens 1968; Staręga 1976; Martens 1978, 2006; Mučalica 1988; Klimeš 2000; Komposch 2002; Komposch \& Gruber 2004; Schönhofer \& Holle 2007). Vertical distribution: 120-1650 m a.s.l. (Komposch

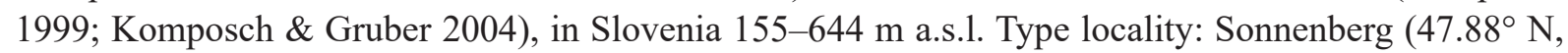
$16.31^{\circ} \mathrm{E}, 480 \mathrm{~m}$ a.s.1.), Leitha Mts, Burgenland, Austria. 
Table 3. Length of appendage-segments in millimeters in N. bidentatum sparsum Gruber \& Martens, 1968 from Prosenjakovci, Slovenia: $\widehat{\partial}($ ( + ).

\begin{tabular}{lccccccc}
\hline & Trochanter & Femur & Patella & Tibia & Metatarsus & Tarsus & Total length \\
\hline Pedipalp & $0.25(0.28)$ & $0.75(0.74)$ & $0.52(0.60)$ & $0.45(0.47)$ & - & $0.29(0.28)$ & $2.26(2.37)$ \\
Leg I & $0.23(0.24)$ & $0.68(0.70)$ & $0.39(0.38)$ & $0.61(0.64)$ & $1.06(1.05)$ & $0.97(0.98)$ & $3.94(3.99)$ \\
Leg II & $0.27(0.31)$ & $1.30(1.56)$ & $0.47(0.45)$ & $1.13(1.01)$ & $2.03(1.68)$ & $0.84(1.47)$ & $5.98(6.48)$ \\
Leg III & $0.19(0.23)$ & $0.87(0.96)$ & $0.36(0.35)$ & $0.61(0.64)$ & $1.15(1.17)$ & $0.88(0.91)$ & $4.06(4.26)$ \\
Leg IV & $0.25(0.25)$ & $1.32(1.21)$ & $0.39(0.44)$ & $0.78(0.89)$ & $1.84(1.84)$ & $1.18(1.16)$ & $5.75(5.79)$ \\
\hline
\end{tabular}

Table 4. Length of appendage-segments in millimeters (trochanters not presented) in $N$. bidentatum sparsum Gruber \& Martens, 1968 from Sonnenberg, Austria: ${ }^{\lambda}$ holotype (ㅇ) (from Gruber \& Martens 1968).

\begin{tabular}{lcccccc}
\hline & Femur & Patella & Tibia & Metatarsus & Tarsus & Total length \\
\hline Leg I & $0.75(0.75)$ & $0.30(0.30)$ & $0.55(0.55)$ & $0.95(0.95)$ & $0.80(0.90)$ & $3.35(3.45)$ \\
Leg II & $1.30(1.25)$ & $0.35(0.40)$ & $1.00(1.00)$ & $1.95(1.95)$ & $1.45(1.45)$ & $6.05(6.05)$ \\
Leg III & $0.75(0.75)$ & $0.30(0.30)$ & $0.50(0.50)$ & $1.05(1.10)$ & $0.60(0.75)$ & $3.20(3.40)$ \\
Leg IV & $1.20(1.20)$ & $0.35(0.30)$ & $0.75(0.75)$ & $1.70(1.70)$ & $1.05(1.00)$ & $5.05(4.95)$ \\
\hline
\end{tabular}

\section{Ecology}

Nemastoma b. sparsum is a lowland and hilly land subspecies inhabiting various types of acidophilous and neutrophilous forests of Fagus sylvatica, Carpinus betulus L. and Quercus petraea (Matt.) Liebl., riverine forests, and thermophilous scrubs in north-eastern Slovenia, mostly on non-calcareous soils. Phenology: adults eurychronous.

Nemastoma relictum Gruber \& Martens, 1968 stat. nov. Komposch Figs 1B, 2, 4C, 5C, 6C, 7D, 8D, 9C, 10C, 11D, 12C, 13C; Tables 5-6

Nemastoma bidentatum relictum Gruber \& Martens, 1968: 147-149, figs 14-16, 24 (original description).

Nemastoma bidentatum relictum - Martens 1978: 112-113, figs 148-150. - Komposch \& Gruber 2004: 485. — Komposch 2009: 476; 2011: 65. — Schönhofer 2013: 36.

\section{Diagnosis}

Species within the $N$. $b$. complex with button-shaped Ch-Apo, and secretion field oriented frontally. PaFe without spines, slightly club-shaped (Pa-Fe min:max width $\sim 1: 2.3$ ). Rec sem with 5 lobate, tubularsaccular vesicles.

\section{Material examined}

AUSTRIA • 2 ठิ $\widehat{~}, 3$ 우; Großes Kar, the Koralpe Mts; 25 Oct. 2005; C. Komposch leg. (CK det.); PMSL.

Description (according to Gruber \& Martens 1968, slightly modified)

Male (from Großes Kar, Salzburg)

BoDy. Length 1.55-1.60.

CheliCERAE. Ch with flat triangular dorsal margin in front of dorsal indentation; ventral margin straight, with minute triangular hump. Ch-Apo button-like, inferior and frontal margins straight, embracing a 
Table 5. Length of appendage-segments in millimeters in N. relictum Gruber \& Martens, 1968 stat. nov. from Großes Kar, Koralpe, Austria: ô ( $(+)$.

\begin{tabular}{lccccccc}
\hline & Trochanter & Femur & Patella & Tibia & Metatarsus & Tarsus & Total length \\
\hline Pedipalp & $0.25(0.25)$ & $0.65(0.63)$ & $0.51(0.47)$ & $0.41(0.37)$ & - & $0.32(0.25)$ & $2.14(1.97)$ \\
Leg I & $0.21(0.23)$ & $0.78(0.74)$ & $0.34(0.40)$ & $0.63(0.52)$ & $0.90(0.90)$ & $0.89(0.76)$ & $3.75(3.55)$ \\
Leg II & $0.20(0.23)$ & $1.23(1.23)$ & $0.42(0.39)$ & $0.98(0.98)$ & $1.82(1.62)$ & $1.21(1.35)$ & $5.86(5.80)$ \\
Leg III & $0.20(0.20)$ & $0.78(0.56)$ & $0.31(0.31)$ & $0.56(0.54)$ & $1.07(1.00)$ & $0.67(0.80)$ & $3.59(3.41)$ \\
Leg IV & $0.24(0.20)$ & $1.13(1.15)$ & $0.39(0.38)$ & $0.83(0.77)$ & $1.56(1.45)$ & $1.12(1.04)$ & $5.27(4.99)$ \\
\hline
\end{tabular}

Table 6. Length of appendage-segments in millimeters (trochanters not presented) in N. relictum Gruber \& Martens, 1968 stat. nov. from the vicinity of Tappenkarsee, Obertauern, Austria: ${ }^{\lambda}$ holotype () (from Gruber \& Martens 1968).

\begin{tabular}{lcccccc}
\hline & Femur & Patella & Tibia & Metatarsus & Tarsus & Total length \\
\hline Leg I & $0.65(0.75)$ & $0.30(0.30)$ & $0.50(0.50)$ & $0.85(0.90)$ & $0.80(0.85)$ & $3.10(3.30)$ \\
Leg II & $1.10(1.15)$ & $0.40(0.40)$ & $0.95(0.90)$ & $1.70(1.65)$ & $1.30(1.30)$ & $5.45(5.40)$ \\
Leg III & $0.70(0.75)$ & $0.30(0.30)$ & $0.50(0.55)$ & $1.00(1.00)$ & $0.75(0.80)$ & $3.25(3.40)$ \\
Leg IV & $1.10(1.15)$ & $0.35(0.30)$ & $0.75(0.75)$ & $1.50(1.50)$ & $1.00(1.00)$ & $4.70(4.70)$ \\
\hline
\end{tabular}

$90^{\circ}$ angle, Ch-Apo dorsally round, secretion field with dense setae (brush) frontally (Figs 4C, 6C, 9C). Proximal article length 0.54 , distal article length 0.53 , max width 0.18 , movable finger length 0.19 .

PEDIPALPS. Pa-Tr dorsal margin high, oval, leaned distally; Pa-Fe distended distally (max:min width $\sim 1.7: 1$ ), without spines. Pa-Pt with conspicuously convex ventral margin and finger-like medial Apo. $\mathrm{Pa}-\mathrm{Ti}$ and $\mathrm{Pa}-\mathrm{Ta}$ straight (Figs 5C, 6C). Pa article lengths in Table 5.

PenIs. Pe length 1.6, Pe basis 1/4 Pe length, glans isosceles triangular (Figs 7C, 8C).

LEGS. Leg article lengths in Tables 5-6.

Female (from Großes Kar, Salzburg)

BoDy. Length 1.70 .

Chelicerae. Ch basal article with low-arched dorsal margin in front of indentation, and ventral lowarched concavity, Ch basal article length:width $\sim 2.3: 1$ (Fig. 13C). Proximal article length 0.56, distal article length 0.55 , max width 0.19 , movable finger length 0.20 .

PediPALPs. Pa short, $\sim 2 \mathrm{~mm}$ long, Pa-Tr with high, isosceles triangular dorsal margin arched on the top, Pa-Fe distended distally (max:min width $\sim 1.6: 1$ ), Pa-Ta short, Pa-Ta length:Pa-Ti length $\sim 1: 1.6$ (Fig. 13C). Pa article lengths in Table 5.

OvIPOsITOR. Rec sem with 5 lobate, tubular-saccular vesicles (Fig. 11D).

LEGS. Leg article lengths in Tables 5-6.

\section{Remarks}

We did not examine the original type material; for the current description, we used other specimens from the type location. Rec sem were found much different from the other taxa within the $N$. b. complex. 


\section{Distribution}

Austria, endemite of eastern Central Alps (Gruber \& Martens 1968; Martens 1978; Komposch 1999, 2009). Vertical distribution: 1325-2240 m a.s.1. (Komposch 1999, 2009). Type locality: Großes Kar, near the Tappenkarsee Lake, Kleinartal, Salzburg $\left(47.19^{\circ} \mathrm{N}, 13.32^{\circ}\right.$ E, $1760 \mathrm{~m}$ a.s.l.), Radstädter Tauern Mts, Austria.

\section{Ecology}

Nemastoma relictum stat. nov. is a montane to alpine species within the $N$. $b$. complex inhabiting forests below the krummholz zone, alpine meadows, spring-areas, and gravel and block heaps in the eastern Central Alps (Gruber \& Martens 1968; Martens 1978; Komposch 1999, 2009) in 1325-2240 m a.s.1. (Komposch 1999). Phenology: evidence of adults from the end of May till the end of October, but probably eurychronous.

Nemastoma pluridentatum (Hadži, 1973) stat. nov. Novak Figs 2, 4D, 5D, 6D, 9D, 10D, 12D; Table 7

Nemastoma (Lugubrostoma) triste pluridentatum Hadži, 1973a: 43, fig 33a (original description) [partim; the description also includes $N$. bidentatum schmidti ssp. nov., $N$. bidentatum gruberi ssp. nov. $\times$ $N$. bidentatum schmidti $\mathrm{ssp}$. nov. and $N$. bidentatum martensi $\mathrm{ssp}$. nov. $\times N$. bidentatum schmidti ssp. nov.].

Nemastoma bidentatum bidentatum - Martens 1978: 107.

Nemastoma ?bidentatum pluridentatum - Novak 2005a: 314, fig. 34.

Nemastoma bidentatum pluridentatum - Schönhofer 2013: 36.

Diagnosis (partly according to Hadži 1973a)

Robust, short-legged member of the Nemastoma b. complex, with extremely stout $\mathrm{Ch}$ and Pa. Ch-Apo small, slightly trapezoid button-like, wider than high, with secretion field in frontal third. Pa-Tr nearly round, Pa-Fe and Pa-Pt very stout, Pa-Fe club-shaped, with 4 large, wide humps.

\section{Material examined}

\section{Holotype}

BOSNIA AND HERZEGOVINA - YK10 • 1 đ̊; Mt. Paljenik, Vlašić planina Mts; 1933 m a.s.l.; 18 Jun. 1962; E. Pretner leg. (4/2005, JH's microscopic preparation); PMSL. (Note: UTM code in Novak (2005a) was erroneously cited as YJ19.)

\section{Partial redescription}

Note: male body and penis missing; female unknown.

Male (holotype)

Chelicerae. Ch basal article length (without Apo) 0.57, stout, 1.8 times as long as wide at $\mathrm{Ch}$ max width at dorsal hump, distally rounded. Ch-Apo rounded low trapezoid, with longest margin distally, secretion field frontally. Distal article length 0.57, max width 0.26, movable finger length 0.28 (Figs 4D, 6D, 9D).

PediPalps. Pa stout, Pa-Fe extremely stout, with 4 large, bulky humps (instead of spines), club-shaped (Pa-Fe min:max width 1:3.6), Pa-Pt extremely stout (length:max width $\sim 1: 2.8$ ) (Figs 5D, 6D). Pa article lengths in Table 7. 
Table 7. Length of appendage-segments in millimeters in N. pluridentatum (Hadži, 1973) stat. nov. from Mt. Paljenik, Bosnia and Herzegovina: $\widehat{o}$ holotype, + unknown.

\begin{tabular}{lccccccc}
\hline & Trochanter & Femur & Patella & Tibia & Metatarsus & Tarsus & Total length \\
\hline Pedipalp & 0.27 & 0.68 & 0.49 & 0.38 & - & 0.29 & 2.11 \\
Leg I & 0.19 & 0.60 & 0.31 & 0.41 & 0.50 & 0.49 & 2.50 \\
Leg II & 0.22 & 1.05 & 0.28 & 1.25 & 1.05 & 0.80 & 4.65 \\
Leg III & 0.21 & 0.70 & 0.25 & 0.50 & 0.65 & 0.65 & 3.16 \\
Leg IV & 0.22 & 0.95 & 0.25 & 0,60 & 1,20 & 0.75 & 3.95 \\
\hline
\end{tabular}

Legs. Pseudoarticle leg-Fe formula I-IV: 0-2-1-2. Tarsomere formula I-IV: 8-13-5-8. Leg article lengths in Table 7.

\section{Remarks}

Under the name Nemastoma (Lugubrostoma) triste pluridentatum, Hadži (1973a) described four taxa: a) N. pluridentatum (Hadži, 1973) stat. nov. (Hadži 1973a: fig. 33a) from Mt. Paljenik (1943 m a.s.l.) in the Vlašić Mts, Bosnia, b) a hybrid N. b. gruberi ssp. nov. × N. b. schmidti ssp. nov. (Hadži 1973a: fig. 33b, č) from the surroundings of Novo mesto, Slovenia, c) a hybrid N. b. martensi ssp. nov. $\times N . b . s c h m i d t i$ ssp. nov. (Hadži 1973a: fig. 33c) from the surroundings of Novo mesto, which Hadži (ibid.) erroneously cited for Mt. Vlašić, and d) Nemastoma b. schmidti ssp. nov. from Mt. Šmarna gora, Slovenia (Hadži 1973a: fig. 34a), from the surroundings of Ljubljana (Hadži 1973a: fig. 34b), and from the cave Jama Sv. Janeza pri Prestranku near Postojna, Slovenia (Hadži 1973a: fig. 34c). Figs 33 and 34 were drawn freehand; consequently, shapes and dimensions only partly correspond to the real measurements. Hadži's original microscopic preparation labeled "Nemastoma bidentatum Roewer pulli, A-1065 (Novo Mesto), legit Karaman" includes two males and a female, but no juveniles. Hadži (ibid.) drew some details of the males, including damaged legs; see remarks on Nemastoma (Stridulostoma) seliskari Hadži, 1973 under $N$. b. martensi ssp. nov. $\times N$. b. schmidti ssp. nov. Given that some important parts, e.g., Pa of one male, are missing, Hadži probably made this incomplete preparation after observation of the specimens under a microscope. In his original paper (Hadži 1973a), the type specimen and the type locality of N. pluridentatum stat. nov. were not designated, but he did that in the catalogue (Hadži 1973b: 13) under a mistyped name: "N. (L.) triste pleuridentatum Hadži, 1973". There he wrote: "Loc. typ.: Novo mesto". Since the specimens from this locality belong to the above-mentioned hybrids, Novo mesto cannot be the type locality of $N$. pluridentatum stat. nov.; that is the first-mentioned location in Bosnia, as fig. 33a (Hadži 1973a) is the only drawing that refers to this taxon. Unfortunately, only the preparation of one $\mathrm{Ch}$, one Pa and four legs of the male holotype are preserved, while the body, as well as a further male and female mentioned by Hadži (1973a: 44) are missing. Because of the lack of any information on the Pe, the saw on Fe IV in preserved legs is the only available character allowing us to place this taxon into the $N$. b. complex, interpreted as $N$. ?bidentatum pluridentatum (Novak 2005a: fig. 34) or N. b. pluridentatum (Schönhofer 2013). In 2019, we visited the type locality Mt. Paljenik, but we did not find further specimens of $N$. pluridentatum. Instead we found a new, much different $N$. kozari sp. nov. there, which unlikely hybridize with $N$. pluridentatum stat. nov. Consequently, we ascribed the species status to $N$. pluridentatum. See remarks under $N$. relictum stat. nov., $N$. kozari sp. nov. and hybrids $N$. b. gruberi ssp. nov. $\times N$. b. schmidti ssp. nov. and $N$. b. martensi ssp. nov. $\times N$. b. schmidti ssp. nov.

\section{Distribution}

Bosnia and Herzegovina, endemic to central Bosnia, probably endemic to the Vlašić Mts. Type locality: Mt. Paljenik in the Vlašić planina Mts, Bosnia, (44.29 ${ }^{\circ} \mathrm{N}, 17.64^{\circ} \mathrm{E}, 1933 \mathrm{~m}$ a.s.l.), Bosnia and Herzegovina. 


\section{Ecology}

Nemastoma pluridentatum stat. nov. inhabit the highest peak areas with the Mastgrass Gentianello crispatae-Nardetum strictae Redžić 1990 communities. Phenology: adults probably eurychronous.

Nemastoma bidentatum gruberi Novak, Slana Novak, Kozel \& Raspotnig ssp. nov. urn:1sid:zoobank.org:act:17EA28A1-5F36-4DFC-9191-9C8680496FAC Figs 1A, C, 2-3, 4E, 5E, 6E, 7E, 8E, 9E, 10E, 11E, 12E, 13D; Table 8

Nemastoma bidentatum sparsum - Novak et al. 2002: 136 [partim: Brežice, Črnomelj, Kočevje, Krško, Litija, Metlika, Novo mesto, Ribnica, Sevnica,Trebnje]. — Novak 2004b: 245 [partim: Buje, Karojba, Vižinada]; 2005b: 105.

\section{Diagnosis}

Relatively long-legged subspecies of Nemastoma bidentatum, with strait Ch ventral margin, and $\mathrm{Pa}-\mathrm{Fe}$ with either 4 subequidistantly positioned spines or pointed tubercles or long setae on low protrusions, or their combination in the distal Pa-Fe half, or alternatively, with 2-3 distal stout thorns. Rec sem with one tubular and 12 elongated balloon-like vesicles.

\section{Etymology}

The subspecies name 'gruberi' is dedicated to Jürgen Gruber (Vienna), our teacher, colleague and friend, who generously introduced us to the taxonomy of harvestmen and helped in identifying problematic specimens.

\section{Material examined}

\section{Holotype}

SLOVENIA - WL18 • 1 ○ं; Dolenje Laknice, Mokronog; $45.93^{\circ}$ N, $15.21^{\circ}$ E; 237 m a.s.1.; 26 Oct. 2013; L. Slana Novak and T. Novak leg.; mixed forest litter sift; PMSL-Opiliones-GR 4661.

\section{Paratypes}

SLOVENIA - WL18 • 1 क ; same collection data as for holotype; PMSL-Opiliones-GR $4664 \cdot 5$ えð, 4 우 ; same collection data as for holotype; PMSL-Opiliones-GR4662, GR4666, GR4667, GR4669, GR4673, GR4676 to GR4679.

\section{Other material}

ITALY - VL05 • 1 क; Chiusa/Ricmanje; 29 Sep. 1999; L. Slana Novak and T. Novak leg. (275/1999, rev. 2015); PMSL. - VL15 • 1 o; Basovizza/Bazovica; 29 Sep. 1999; L. Slana Novak and T. Novak leg. (271/1999, rev. 2015); PMSL.

CROATIA - UL92 • $3 \widehat{\jmath} \widehat{\partial}, 2$ q $q$ (paratypes of N. b. sparsum); vicinity of Buje, and VL02, Vižinada; Sep. 1963; R. Sturany leg.; NHMW 4700. - VL01 • 1 đ̇; Karojba; 28 Feb. 1990; F. Potočnik leg. (1307/1998, rev. 2018); PMSL. - VL13 - 1 क; Mlini; 30 Sep. 1990; L. Slana Novak and T. Novak leg. (32/1990, rev. 2015); PMSL. - XL90 • 1 đ, 2 † o ; Ponor Sušik, Drežnik; 8 Sep. 2009; A. Schönhofer leg. (Coll. ASc 334, ASc det., TN rev. 5/2012); PMSL.

SLOVENIA - UL93 • 4 q $q, 1$ juv.; Abrami; 19 Jul. 1999; L. Slana Novak and T. Novak leg. (148/1999, rev. 2018); PMSL • 2 o o ; ibid.; 20 Jul. 1999; L. Slana Novak and T. Novak leg. (188/1999, rev. 2015); PMSL • 1 \%; Dragonja; 14 Sep. 2000; L. Slana Novak and T. Novak leg. (154/2001, rev. 2015); PMSL

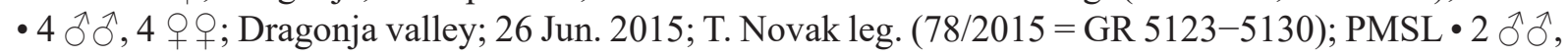
3 우; Ivankovec; 23 Sep. 1998; L. Slana Novak and T. Novak leg. (1791/1998, rev. 2018); PMSL・ 1 ㅇ; 
Lucija-Seča - Debernardo; 22 Sep. 1998; L. Slana Novak and T. Novak leg. (1817/1998, rev. 2019); PMSL 1 đ̊; Korte; 23 Sep. 1998; L. Slana Novak and T. Novak leg. (1821/1998, rev. 2018); PMSL・ 33 วิ ô, 30 우; Medljan, Korte; 21 Aug. 1999; T. Novak leg. (239/1999, rev. 2018); PMSL・1 juv.; ibid.; 22 Jun. 2000; B. Bertoncelj, B. Ogrizek and T. Novak leg. (217/2000, rev. 2018); PMSL • 1 क; ibid.; (218/2000, TN rev. 2019); PMSL・1 ठ̊; Padna; 19 Jul. 1999; L. Slana Novak and T. Novak leg. (168/1999, rev. 2017); PMSL • 2 우; Parecag; 23 Sep. 1998; L. Slana Novak and T. Novak leg. (1788/1998, rev. 2018); PMSL • 1 q; Seča; 10 Oct. 1998; L. Slana Novak and T. Novak leg. (1844/1998, rev. 2008); PMSL - 1 ô, 1 क ; ibid.; 24 Oct. 1998, L. Slana Novak and T. Novak leg. (1881/1998, rev. 2007); PMSL • 3 우;

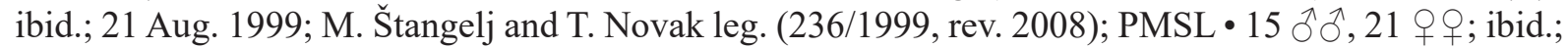
21 Aug. 1999; M. Štangelj and T. Novak leg. (231/1999, rev. 2015); PMSL・ 1 ð, 6 우 or; Sečovlje; 1 Mar. 2009; L. Slana Novak and T. Novak leg. (1/2015); PMSL・ 1 đ; Sv. Peter; 11 Oct. 1998; L. Slana Novak

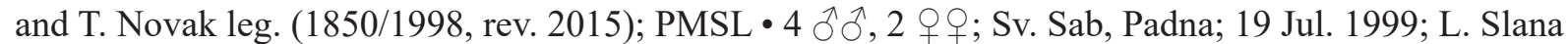
Novak and T. Novak leg. (151/1999, rev. 2009); PMSL • 1 क; Škrline, Dragonja; 20 Jul. 1999; L. Slana Novak and T. Novak leg. (194/1999, rev. 2015); PMSL. - VL03 • 2 ふ઼ ô, 1 ᄋ ; Boršt; 14 Apr. 2011; T. Novak leg. (76/2011, rev. 2015); PMSL • 2 o 9 ; ibid.; (81/2011, rev. 2015); PMSL. - VL04 • 1 ㅇ; Ankaran; 29 Sep. 1999; L. Slana Novak and T. Novak leg. (276/1999, rev. 2015); PMSL・ 1 O, 1 q; Kolombini, Spodnje Škofije; 26 Sep. 2007; L. Slana Novak and T. Novak leg. (94/2007, rev. 2018); PMSL

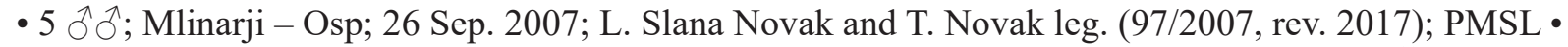
1 ก̂, 1 क; Rižana; 24 Sep. 2016; L. Slana Novak and T. Novak leg. (18/2018); PMSL • 1 q; Škocjanski zatok, Koper; 15 Oct. 2009; S. Polak leg. (PK 1/2015); NM・ 4 ổ, 3 q o+; Zgornje Škofije - Tinjan; 26 Sep. 2007; L. Slana Novak and T. Novak leg. (88/2007); PMSL. - VL07 • 4 q $O$; Hrastje, Šmarje; 17 Aug. 2000 (674/2000, rev. 2015); PMSL. - VL13 • 1 đ̊; Poletiči, Kubed; 4 Sep. 2016; T. Marinko leg. (27/2016); PMSL • 1 J, 1 क; Sočerga; 28 Sep. 1990; L. Slana Novak and T. Novak leg. (31/1990,

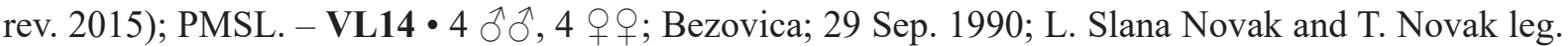
(LSN 1/1990, TN rev. 2018); PMSL • 1 ô; Hrpelje, Kozina; 2 Apr. 1993; S. Brelih leg. (651/1998, rev. 2018); PMSL・ 2 ठో ; Predloka; 29 Sep. 1990; L. Slana Novak and T. Novak leg. (35/1990, rev. 2015);

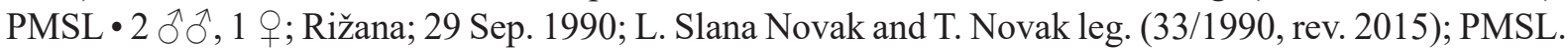
-VL15 • 1 q; Botač; 22 Mar. 1990; M. Černila, B. Horvat and I. Sivec leg. (1246/1997); PMSL. - VL16

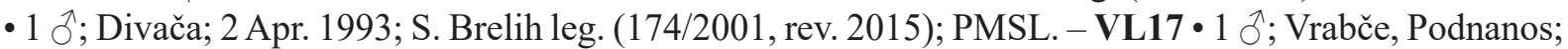
10 Mar. 1994; S. Brelih leg. (632/1998, rev. 2017); PMSL. - VL18 • 1 ð’; Uhanje; 3 Sep. 2001; T. Novak leg. (329/2001, rev. 2018); PMSL. - VL23 • 1 Õ, 1 क; Poljane pri Podgradu; 18 Aug. 2011; L. Slana Novak and T. Novak leg. (166/2012, rev. 2019); PMSL. - VL25 • 3 우; Buje; 10 Mar. 1994; S. Brelih leg. (163/2001, rev. 2018); PMSL・ 1 § , 2 우; Goriče pri Framljah; L. Slana Novak and T. Novak leg. (343/2011); PMSL • 1 o; ibid.; (344/2011); PMSL. - VL28 • 1 ô, 1 o; Podvrh, Raka; 18 Jul. 2012;

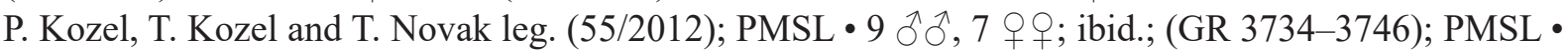
3 우; ibid.; (GR 3791); PMSL. - VL29 • 6 ỗ 0 , 12 우; Dolnje Brezovo, Sevnica; 30 Jun. 2012; (GR 3670-3683); PMSL. - VL36 • 1 Jे, 1 क; Palčje - Mt. Javorniki; 1 Oct. 2011; L. Slana Novak and T. Novak leg. (361/2011, rev. 2019); PMSL. - VL45 • 1 क; Koritnice - Mašun; 2 Oct. 2011; L. Slana Novak and T. Novak leg. (329/2011, rev. 2014); PMSL. - VL47 • 1 क; Suha reber, Koritnice; 21 Jan. 2007; S. Polak leg. (107/2007); NM. - VL54 • 1 +; Sviščaki; 20 May 1989; T. Trilar leg. (271/2000, rev. 2017); PMSL • 1 क; ibid.; 25 May 1989; T. Trilar leg. (275/2000, rev. 2017); PMSL • 1 क; ibid.; 21 Jul. 2001; B. Bertoncelj, U. Bertoncelj and T. Novak leg. (29/2001, rev. 2018); PMSL • 1 क; ibid.; (34/2001, rev. 2011); PMSL・ 1 đ, 3 우; Travni Dolci, Mt. Snežnik; 12 Aug. 2001; L. Slana Novak and T. Novak leg. (195a/2001, rev. 2020); PMSL・ 2 ô ô, 4 우우 Mt. Veliki Snežnik; 20 Jul. 1999; L. Slana Novak and T. Novak leg. (185a/1999, rev. 2020); PMSL・1 Ō; ibid.; 12 Sep. 2018; L. Slana Novak and T. Novak leg. (116/2018); PMSL. - VL36 • 1 đ, 1 q; Palčje - Javorniki; L. Slana Novak and T. Novak leg. (361/2011, rev. 2015); PMSL. - VL66 • 1 o+; Benete, Runarsko; 31 May 2004; L. Slana Novak and T. Novak leg. (33/2004, rev. 2015); PMSL. - VL67 • 5 §ð, 3 q ; Nova vas - Volčje; 26 Sep. 2006; L. Slana Novak and T. Novak leg. (25/2006, rev. 2019); PMSL • 1 đ̊; Runarsko; 26 Sep. 2006; L. Slana Novak and T. Novak leg. (38/2006, rev. 2015); PMSL・ 1 đ, 1 क; Veliki vrh, Runarsko; 26 Sep. 2006; 
L. Slana Novak and T. Novak leg. (36/2006, rev. 2015); PMSL・ 7 đð, 5 우우 Volčje; 26 Sep. 2006; L. Slana Novak and T. Novak leg. (28/2006, rev. 2015); PMSL. - VL69 • 1 \%; Veliki vrh pri Šmarju; 10 May 2008; L. Slana Novak and T. Novak leg. (156/2011); PMSL. - VL74 • 1 đ, 1 o; Mt. Goteniški Snežnik; 28 May 2000; L. Slana Novak and T. Novak leg. (247/2000, rev. 2007); PMSL • 1 q; Strma reber, Zgornji Čačiči; 27 Sep. 1996; L. Slana Novak and T. Novak leg. (LSN 126/1996, TN rev. 2008);

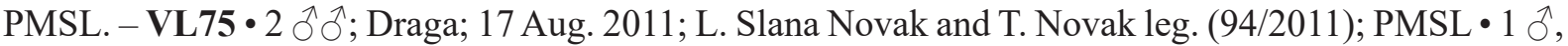
2 qo+; Mt. Goteniški Snežnik; 28 May 2000; L. Slana Novak and T. Novak leg. (253/2000, rev. 2007); PMSL. - VL76 • 1 đ̊; Bukovica, Ribnica; Jul.1994; B. Oven leg. (LSN 25/1996, TN rev. 2009); PMSL

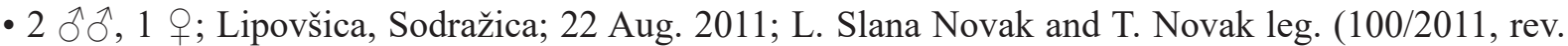
2018); PMSL • 1 đ;; Nova Štifta; 22 Aug. 2011; L. Slana Novak and T. Novak leg. (105/2011); PMSL・

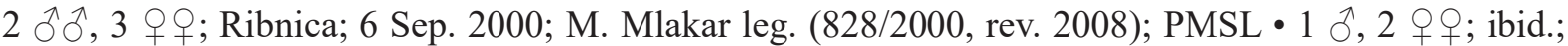
(831/2000, rev. 2019); PMSL • 1 क; Mt. Turn; 27 Jun. 1980; B. Drovenik leg. (383/2002, rev. 2018);

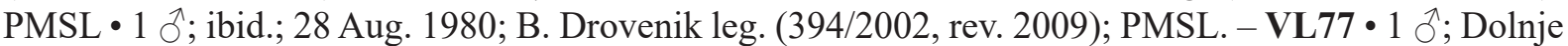
Retje; 3 May 2008; L. Slana Novak and T. Novak leg. (161/2011); PMSL • 1 q; Rašica, Velike Lašče; 16 Jun. 1986 (261/2002, rev. 2015); PMSL・ 1 ô, 1 q; Velike Poljane, Ortnek; 3 May 2008; L. Slana Novak and T. Novak leg. (142/2011, rev. 2019); PMSL. - VL78 • 1 ô; Laporje; 3 May 2008; L. Slana Novak and T. Novak leg. (151/2011); PMSL • 1 क ; Zagradec pri Grosupljem; 7 Jul. 1986; L. Slana Novak and T. Novak leg. (500/2002, rev. 2015); PMSL. - VL79 • 1 क; Veliki Lipoglav - Dobrinje; 8 Jul. 1986; L. Slana Novak and T. Novak leg. (467/2002, rev. 2015); PMSL. - VL83 • 2 đ̂đ 24 Apr. 2001; S. Brelih leg. (121/2001, rev. 2019); PMSL. - VL84 • 1 §ో; Jelenja vas; 3 May 2008;

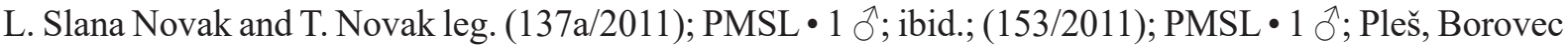
pri Kočevski Reki; 19 Sep. 2013; P. Kozel and T. Novak leg. (71/2013, rev. 2018); PMSL. - VL85 • 1 \%; Grčarice; 18 Oct. 2013; T. Novak leg. (63/2013, rev. 2015); PMSL・ 1 ð, 2 우; Grčarske Ravne; 18 Oct. 2013; T. Novak leg. (66/2013, TN rev. 2019); PMSL • 3 우; Kočevje; 6 Sep. 2000; M. Mlakar leg.

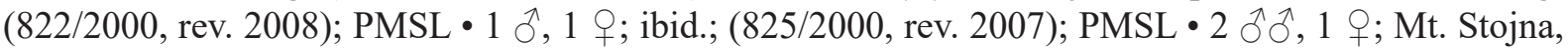
Kočevje; 25 Oct. 1996; S. Brelih leg. (92/2001, rev. 2008); PMSL. - VL86 • 1 P; Dolenja vas; 3 May 2008; L. Slana Novak and T. Novak leg. (110/2011); PMSL • 1 Ô, 1 q; ibid.; (154/2012, rev. 2018); PMSL. - VL87 • 1 đ; Breg pri Zagradcu; 4 Nov. 1993; S. Brelih leg. (805/1998, rev. 2007); PMSL. VL89 • 1 क; Potok, Stična; 4 Mar. 1994; S. Brelih leg. (658/1998, rev. 2015); PMSL. - VL93 • 1 đ;; Brsnik, Fara; 3 May 2008; L. Slana Novak and T. Novak leg. (157/2011, rev. 2018); PMSL. - VL94 • 1 ภิ, 1 o; Dolenja Žaga; 29 Apr. 2001; S. Brelih leg. (131/2001, rev. 2015); PMSL・ 1 q; Dolenja Žaga - Podstene; 26 Jun. 1999; L. Slana Novak and T. Novak leg. (90/1999, rev. 2008); PMSL・ 1 O, 1 O ; Gorenja Žaga; 3 May 2008; L. Slana Novak and T. Novak leg. (111a/2011); PMSL・ 1 ô, 1 ᄋ; ibid.; (159/2011); PMSL • 1 9; Ograja; 3 May 2008; L. Slana Novak and T. Novak leg. (109a/2011); PMSL・

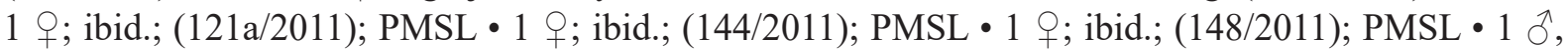
1 q; Ograja - Suhor - Morava; 3 May 2008; L. Slana Novak and T. Novak leg. (120a/2011); PMSL •

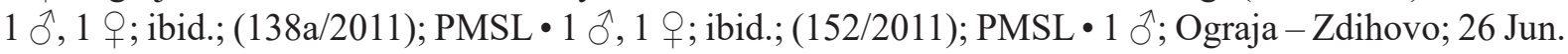
1999; L. Slana Novak and T. Novak leg. (76/1999, rev. 2008); PMSL • 3 juvs; ibid.; 12 Jun. 1999; L. Slana Novak and T. Novak leg. (32/1999, rev. 2008); PMSL • 2 juvs; Podstene pri Kostelu; 12 Jun. 1999; L. Slana Novak and T. Novak leg. (24/1999, rev. 2008); PMSL・ 1 đ̊; Rajndol; 21 Mar. 1994; S. Brelih

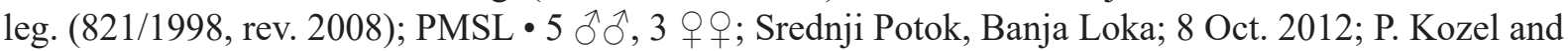
T. Novak leg. (68/2015); PMSL・ 1 đ, 1 क , 2 juvs; Štalcerski preval; 12 Jun. 1999; L. Slana Novak and T. Novak leg. (33/1999, rev. 2008); PMSL. - VL95 • 1 đ̃, 2 q ; ; Dolga vas; 17 Aug. 1985; T. Novak, M. Slana Novak and L. Slana Novak leg. (LSN 97/1986, TN rev. 2007); PMSL・ 1 o, 1 क; Livold; 3 May 2008; L. Slana Novak and T. Novak leg. (123a/2011); PMSL・ 1 Oे, 1 q ; ibid.; (158/2011); PMSL. - VL97 - 1 o; Dvor, Lašče; 10 May 2008; L. Slana Novak and T. Novak leg. (112/2011); PMSL・ 1 o, 2 q ; Železno, Dobrnič; 4 Nov. 1993; S. Brelih leg. (812/1998, rev. 2007); PMSL. - VL99 • 3 $q$; Nova gora, Čatež; 15 Aug. 2007; L. Slana Novak and T. Novak leg. (61/2007, rev. 2015); PMSL. - VM80 • 1 đ; Litija; 10 Sep. 2000; M. Mlakar leg. (809/2000, rev. 2008); PMSL・ 1 ð̊, 1 क; ibid.; (834/2000, rev. 2015); PMSL. - WL03 • 2 ổ; Dolenje Kozice; 18 Apr.-13 May 2002; B. Drovenik and A. Gergeli leg. 
(509/2018); PMSL • 1 ð’; ibid.; 18 Apr.-13 May 2002; B. Drovenik and A. Gergeli leg. (550/2018); PMSL・ 1 đ̊; ibid.; 5 Jul. 2002; B. Drovenik and A. Pirnat leg. (1135/2018); PMSL • 1 क; ibid.; 6 Aug. 2002; B. Drovenik and A. Pirnat leg. (1090/2018); PMSL • 1 क; ibid.; 30 Sep. -28 Oct. 2002; B. Drovenik and A. Gergeli leg. (PK 64/2012); PMSL - 1 q; ibid.; 25 Nov. 2002; B. Drovenik and A. Pirnat leg. (270/2018); PMSL • 2 q ; ibid.; 25 Nov. -23 Dec. 2002; B. Drovenik and A. Gergeli leg. (1027/2018); PMSL・ 4 $\widehat{\jmath}, 5$ 우; Srednji Radenci; 17 Aug. 1985; L. Slana Novak, M. Slana Novak and T. Novak leg. (LSN 51/1986, rev. 2008); PMSL・ 3 ડ̄ $\widehat{~}, 4$ 우; Stari trg ob Kolpi; 21 Mar. 1994; S. Brelih leg. (851/1998, rev. 2008); PMSL • 5 ふึ L. Slana Novak and T. Novak leg. (135a/2011); PMSL・ 5 ふึ - 1 Ĵ, 3 우, 1 juv.; Črmošnjice; 16 Aug. 1985; L. Slana Novak, M. Slana Novak and T. Novak leg. (160/1997); PMSL • 1 q; Koprivnik, Kočevski Rog; 21 Mar. 1994; S. Brelih leg. (867/1998, rev. 2008);

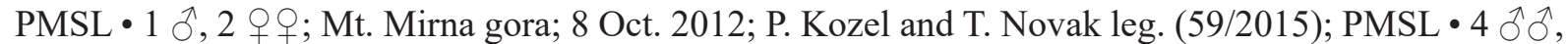
4 ㅇ; ; ibid.; 8 Oct. 2012; P. Kozel and T. Novak leg. (66/2015); PMSL. - WL06・1 Ô; Cerovec - Selišče, Podturn pri Dolenjskih toplicah; 10 May 2008; L. Slana Novak and T. Novak leg. (145/2011, rev. 2017); PMSL. - WL07 • 1 đ̊; Boršt pri Dvoru; 10 May 2008; L. Slana Novak and T. Novak leg. (113/2012); PMSL • 1 P; Prečna, Mirna; Jun. 2000; V. Jaćimović leg. (PK and TN 100/2020); PMSL. - WL08 • 1 万̃; Rdeči kal, Dobrnič; 4 Nov. 1993; S. Brelih leg. (872/1998, rev. 2007); PMSL • 1 ô, 1 O; Trebnje; 29 Aug. 2000; M. Mlakar leg. (787/2000, rev. 2018); PMSL・ 1 đ̄; ibid.; (787a/2000, rev. 2018); PMSL・ 1 O;; ibid.; (788/2000, rev. 2008); PMSL. - WL13 • 4 q ; ; Balkovci, Vinica; 15 Aug. 1999; M. Štangelj and T. Novak leg. (212/1999, rev. 2008); PMSL・ 2 ภึ, 2 우; Dolenji Radenci - Breg pri Sinjem vrhu;

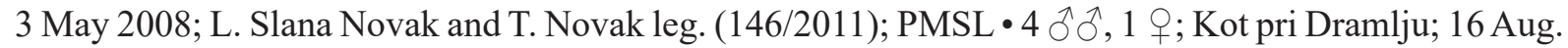
1999; M. Štangelj and T. Novak leg. (207/1999, rev. 2015); PMSL • 3 $\widehat{\jmath}, 2$ q $q$; Špeharji, Vinica;

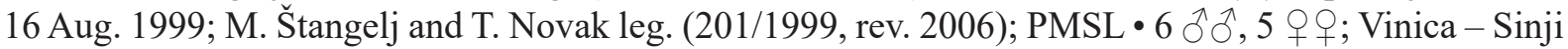
vrh; 17 Aug. 1985; L. Slana Novak, M. Slana Novak and T. Novak leg. (LSN 59/1986, TN rev. 2007);

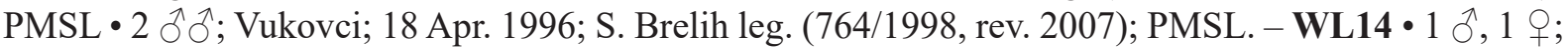
Bistrica, Črnomelj; 26 Jun. 1999; L. Slana Novak and T. Novak leg. (65/1999, rev. 2017); PMSL・ 1 क;

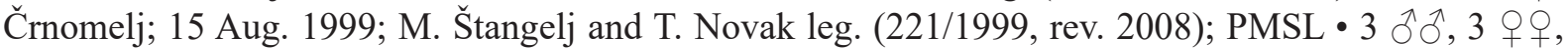
2 juvs; Desinec - Črnomelj; 17 Aug. 1985; L. Slana Novak, M. Slana Novak and T. Novak leg. (385/1998,

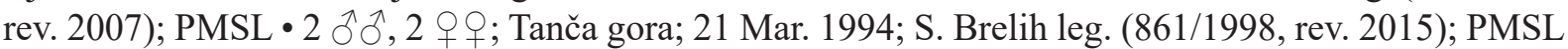

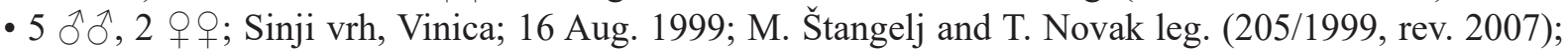

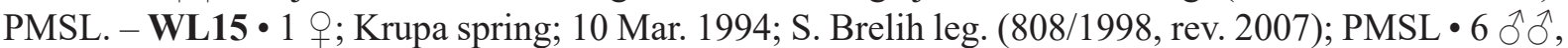
3 우, 3 juvs; Lokve, Črnomelj; 2 Jul. 1995; L. Slana Novak and T. Novak leg. (105/1997, rev. 2009);

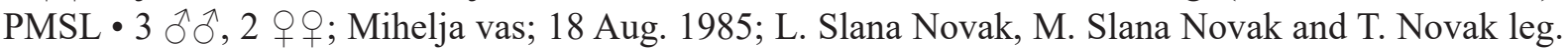
(LSN 55/1986, TN rev. 2017); PMSL • 1 q; Praprot; 6 Aug. 1997; T. Novak leg. (418/1998, rev. 2008); PMSL • 1 q; ibid.; (432/1998, rev. 2008); PMSL • 1 q; ibid.; 22 Jul. 1999; L. Slana Novak and T. Novak

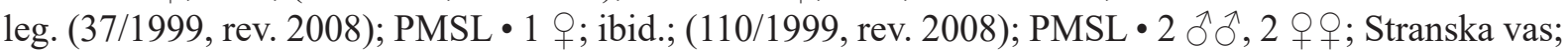

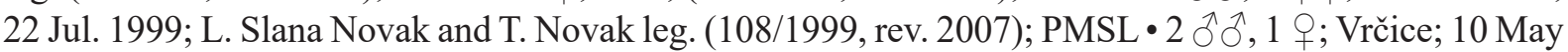
2008; L. Slana Novak and T. Novak leg. (110/2012); PMSL・ 1 ภ, 3 우; Vrčice - Semič; 8 Oct. 2012; P. Kozel and T. Novak leg. (64/2015); PMSL. - WL16 • 2 + ; ; Laze, Uršna Sela; 10 May 2008; L. Slana Novak and T. Novak leg. (103/2012, rev. 2015); PMSL • 1 क; ibid.; (108/2012, rev. 2018); PMSL. WL17 • 1 đ, 1 क; Brod, Novo Mesto; 31 Aug. 2000; M. Mlakar leg. (811/2000, rev. 2007); PMSL・ 1 đ;; Novo mesto; Karaman leg. (87/1983 microscopic preparation, JH det., TN rev. 2011); PMSL・ 1 $\widehat{\partial}, 1$; ibid.; Karaman leg. (108/1983 microscopic preparation, JH det., TN rev. 2011); PMSL • 1 P; ibid.; (814/2000); PMSL • 1 ô; ibid.; (153/2018 microscopic preparation, JH det., TN rev. 2018); PMSL • 1 \%,

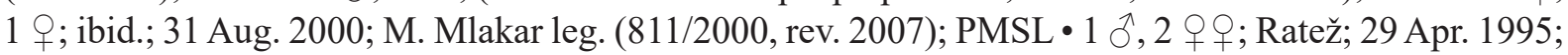

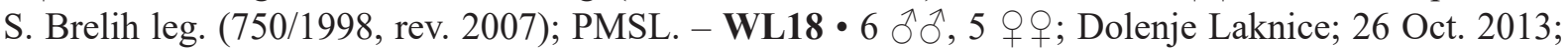
T. Novak leg. (GR 4661, 4662, 4664, 4666, 4667, 4669, 4673, 4676, 4677-4679); PMSL. - WL19 • 1 \%; Boštanj - Mokronog - Križ; L. Slana Novak and T. Novak leg. (70/2007, rev. 2011); PMSL・ 1 đ̊; Jelovec; 15 Aug. 2007; L. Slana Novak and T. Novak leg. (56/2007); PMSL. - WL23 • 1 ð̊; Zilje; 18 Apr. 1996; S. Brelih leg. (767/1998, rev. 2007); PMSL・1 Ô, 2 우; Zilje - Vinica; 17 Aug. 1985; L. Slana Novak, 
M. Slana Novak and T. Novak leg. (364/1998, rev. 2007); PMSL. - WL24 • 5 $\widehat{\partial}, 1$,+ 1 juv.; Gorenjci pri Adlešičih; 15 Aug. 1999; M. Štangelj and T. Novak leg. (218/1999, rev. 2007); PMSL・1 ठో; Dragoši,

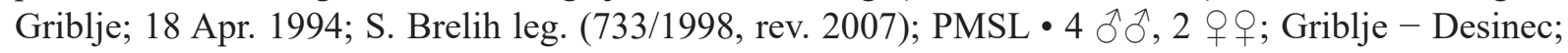
16 Aug. 1985; L. Slana Novak, M. Slana Novak and T. Novak leg. (161/1997, rev. 2009); PMSL. - WL25 - 1 Ō; Bečka jama cave, Cad. No. 6275, Boldraž; 9 Jun. 2004; V. Germovšek, T. Tkavc, A. Kocuvan and T. Novak leg. (21/2004, rev. 2007); PMSL・ 1 đ̃; Božakovo; 3 May 2004; L. Slana Novak and T. Novak

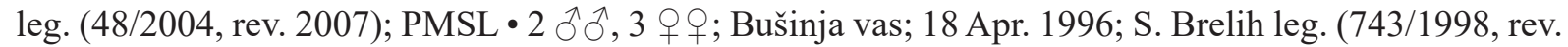
2007); PMSL・ 1 đ, 4 ㅇ; Kapljišče; 16 Aug. 1985; L. Slana Novak, M. Slana Novak and T. Novak leg. (LSN 113/1986, TN rev. 2007); PMSL・ 2 $\widehat{\jmath}, 1$ juv.; ibid. (LSN 119/1986, TN rev. 2007); PMSL・ 3 우; Metlika; 31 Aug. 2000; M. Mlakar leg. (816/2000, rev. 2007); PMSL・ 1 ठ̊; ibid.; (818/2000, rev. 2015); PMSL・ 7 ô ô, 9 우; Trnovec, Metlika; 23 Jul. 1999; L. Slana Novak and T. Novak leg. (141/1999, rev. 2008); PMSL・ 1 đ̃, 2 우; Sv. Urban, Metlika; 18 Apr. 1996; S. Brelih leg. (784/1998, rev. 2007); PMSL. - WL26 • 1 O ; Krašnji vrh; 9 Jun. 2004; V. Germovšek, T. Tkavc, A. Kocuvan and T. Novak leg. (37/2004, rev. 2007); PMSL • 1 đ̊; Markučev Mlin, Krašnji vrh; 1 Jul. 2007; L. Slana Novak and T. Novak leg. (21/2007); PMSL・ 4 우; ibid.; (17/2007, rev. 2011); PMSL. - WL27・ 1 ơ; Gabrje, Novo Mesto; 11 Apr. 1984; I. Sivec leg. (875/1997, rev. 2018); PMSL • 1 क; Gabrje - Sv. Miklavž - Krvavi kamen, Gorjanci Mts; 22 Jul. 1999; L. Slana Novak and T. Novak leg. (123/1999, rev. 2019); PMSL・ 5 ठิ ô,, 6 우; Glažarica, Mt. Gorjanci; 22 Jul. 1999; L. Slana Novak and T. Novak leg. (118/1999, rev. 2015); PMSL・ 1 Ō, 1 q; ibid.; (134/1999, rev. 2018); PMSL・ 1 क; Golo, Šentjernej; 29 Apr. 1995; S. Brelih leg. (757/1998, rev. 2007); PMSL • 1 क ; Sv. Miklavž, Gorjanci Mts; 22 Jul. 1999; L. Slana Novak and T. Novak leg. (128/1999, rev. 2019); PMSL. - WL28 • 3 ふぇ, 7 우; Brezje pri Raki; 27 Jul. 2012; S. Samu, P. Kozel and T. Novak leg. (115/2012); PMSL・1 ô, 1 क; Podvrh, Raka; 18 Jul. 2012; P. Kozel,

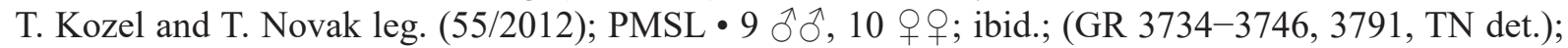

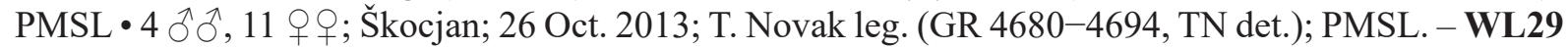
- 3 ठิ $\widehat{~}$, 1 juv.; Boštanj; 5 Jul. 1987; L. Slana Novak, M. Slana Novak and T. Novak leg. (433/1998, rev.

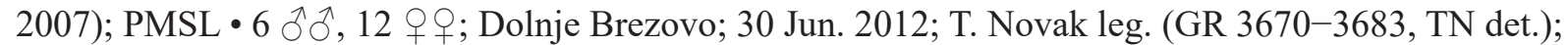
PMSL・ 1 Õ, 1 क ; Sevnica; 29 Aug. 2000; M. Mlakar leg. (785/2000, rev. 2009); PMSL. - WL37 • 1 ô,

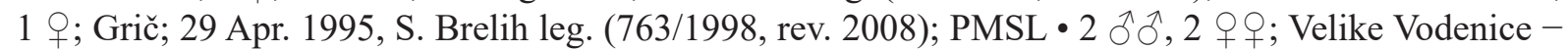
Opatova gora; 15 Aug. 1985; L. Slana Novak, M. Slana Novak and T. Novak leg. (144/1997, rev. 2015); PMSL. - WL38 • 3 o 9 ; Krakovski gozd, Sajevce; 5 May 1993; S. Brelih leg. (792/1998, rev. 2015); PMSL・ 68 ô ô, 59 우; Dolenje Radulje - Raški vrh; 5 Sep. 2012; P. Kozel and T. Novak leg. (PK 98/2012);

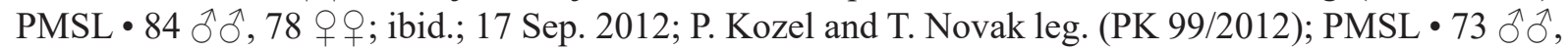

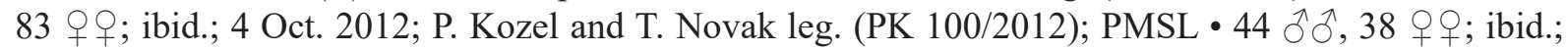
29 Apr. 2013; P. Kozel and T. Novak leg. (PK 43/2013); PMSL・ 74 $\widehat{\jmath}, 71$ q; Podulce - Zabukovje pri Raki; 20 Aug. 2012; P. Kozel and T. Novak leg. (PK 96/2012); PMSL. - WL39 • 1 ô, 1 क ; Arto,

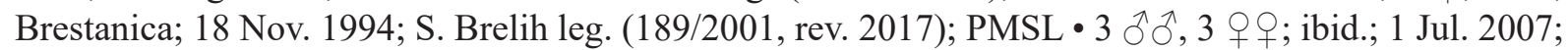
L. Slana Novak and T. Novak leg. (28/2007); PMSL・ 5 ổ, 3 우; Kremen; 12 Oct. 2005; L. Slana Novak and T. Novak leg. (141/2005, rev. 2008); PMSL • 1 O ; Krško; 11 Apr. 1978 (42/2019); PMSL •

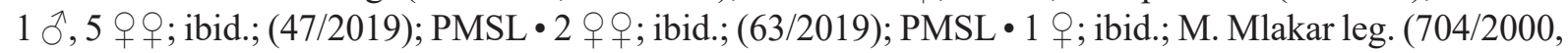

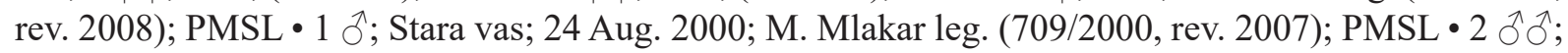

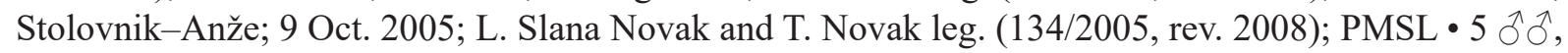
7 qo ; ibid.; 14 Oct. 2005; L. Slana Novak and T. Novak leg. (154/2005, rev. 2007); PMSL. - WL48 • 1 ડ̄; Brežice; 30 Aug. 2000; M. Mlakar leg. (794/2000, rev. 2008); PMSL・ 1 ô; ibid.; 30 Aug. 2000; M. Mlakar leg. (792/2000, rev. 2007); PMSL• 1 क; Vitovec, Velike Malence; Jul. 1994; M. Potokar leg.

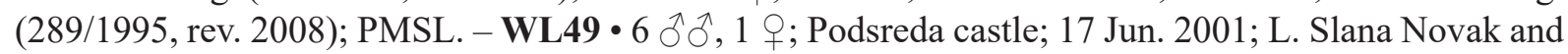
T. Novak leg. (145/2001, rev. 2008); PMSL. - WL59 • 3 ふふ઼, 4 우; Stara vas; 14 Aug. 1985; L. Slana Novak, M. Slana Novak and T. Novak leg. (141/1997, rev. 2007); PMSL • 1 đ̇; ibid.; 24 Aug. 2000; M. Mlakar leg. (709/2000, rev. 2007); PMSL・ 1 đ; ibid.; (714/2000, rev. 2007); PMSL・ 1 §; ibid.; (716/2000, rev. 2007); PMSL. - WM01 • 3 q o ; Hrastnik; 30 Aug. 2000; M. Mlakar leg. (802/2000, rev. 2018); PMSL • 2 + $O$; ibid.; (806/2000, rev. 2018); PMSL. - WM10 • 1 ô, 1 + ; Lokavec, Zidani most; 
26 May 2004; A. Kapla leg. (56/2004, rev. 2007); PMSL • 2 qo; Radeče; 29 Aug. 2000; M. Mlakar leg. (796/2000, rev. 2015); PMSL • 1 q; ibid.; (801/2000, rev. 2015); PMSL. - WM20 • 1 ô; Mt. Lisca; 26 May 2004; A. Kapla leg. (54/2004, rev. 2007); PMSL・ 1 ○; ibid.; (62/2004, rev. 2007); PMSL. WM30・ 7 ふぶ, 8 q,+ 1 juv.; Šentvid pri Planini; 14 Aug. 1983; L. Slana Novak and T. Novak leg. (LSN 52/1983, TN rev. 2007); PMSL・10 $\widehat{\jmath}, 4$ 우; Mt. Bohor; 20 Aug. 1994, L. Slana Novak and T. Novak leg. (66/1997, rev. 2008); PMSL • 3 $\widehat{\partial} \widehat{\partial}, 2$ 우; Tovornik, Mt. Bohor; 13 Jul. 2002; L. Slana Novak and T. Novak leg. (1134/2002, TN rev. 2007); PMSL・ 1 ð, 1 क; ibid.; (1149/2002, rev. 2007);

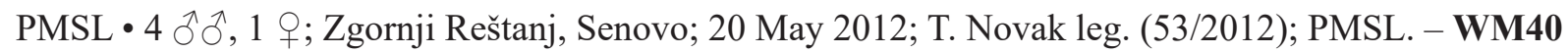
- 1 đ̃; Bistri graben, Kozje; 11 Jul. 2003; T. Novak leg. (160/2003, rev. 2007); PMSL • 2 q ; ibid.;

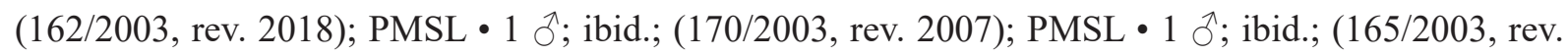

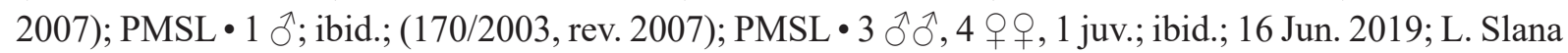
Novak and T. Novak leg. (6/2019); PMSL・ 1 Oे, 1 juv.; ibid.; (11/2019); PMSL・ 1 P; ibid.; (12/2019);

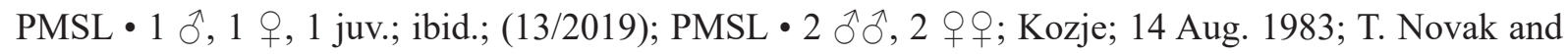
L. Slana Novak leg. (LSN 72/1983, TN rev. 2011); PMSL • 1 क; ibid.; 31 Jul. 1995; S. Polak leg.

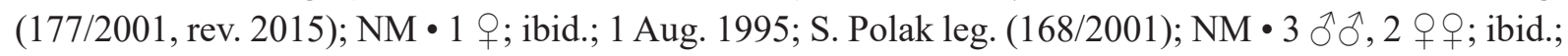
13 Jul. 2002; L. Slana Novak and T. Novak leg. (1150/2002, rev. 2007); PMSL・ 3 $\partial \hat{\jmath}, 5$ $q$ q ; Podsreda; 14 Aug. 1983; T. Novak and L. Slana Novak leg. (LSN 71/1983, TN rev. 2007); PMSL・ 2 ふぇ, 3 우웅 Stara Glažuta; 13 Jul. 2002; L. Slana Novak and T. Novak leg. (1145/2002, rev. 2007); PMSL. - WM41 - 1 Ĵ, 1 O;; Podčetrtek; 10 Aug. 1996; L. Slana Novak and T. Novak leg. (64/1997, rev. 2018); PMSL.

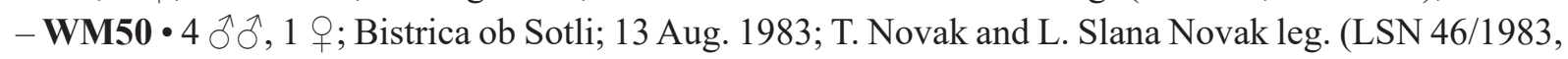
TN rev. 2007); PMSL・ 1 đ, 1 क; Svete gore; 17 May 1997; L. Slana Novak and T. Novak leg. (2/1997,

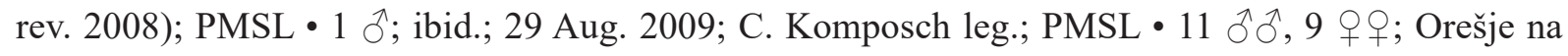
Bizeljskem; 10 Aug. 1996; L. Slana Novak and T. Novak leg. (82/1997, rev. 2009); PMSL.

\section{Description}

Male (holotype)

BoDy. Length 1.99, body width 1.48 .

Chelicerae. Ch basal article (without Apo) length 0.60, relatively long, 2.4 times as long as wide at $\mathrm{Ch}$ max width at dorsal hump. Ch-Apo small, 0.3 times as high as basal article long, rounded-trapezoid, widest distally and highest posteriorly; Ch-Apo 0.18 high, max width 0.17 , min width 0.10 (at the base), ventral margin straight, with 0.70 long and 0.02 deep indentation with 10-20 $\mu \mathrm{m}$ high tipped tubercles and denticles constituting a saw. Secretion field fronto-medially, apically. Distal article length 0.67 , max width 0.24 , movable finger length 0.29 (Figs 4E, 6E, 9E).

PediPALPS. Pa-Fe relatively thin (Pa-Fe min:max width $\sim 1: 2.8$ ), with variable armament: either (the main population) with 4 subequidistant tubercles, spines or long setae, or their combination in the distal Pa-Fe half, or alternatively (marginal population in SW Slovenia), with 2-3 distal stout thorns. Pa-Pt relatively thin (length:max width 1:4.2) (Figs 5E, 6E, 10E). Pa article lengths in Table 8.

Penis. Pe length 1.57, base 0.57, glans 0.18, stylus 0.07 (Figs 7E, 8E).

LEGS. Pseudoarticle leg-Fe formula I-IV: 0-1-1-2. Tarsomere leg-Fe formula I-IV: 10-16-9-10. Leg article lengths in Table 8.

\section{Female}

BoDy. Body length 2.49, body width 1.76. 
Table 8. Length of appendage-segments in millimeters in N. bidentatum gruberi Novak, Slana Novak, Kozel \& Raspotnig ssp. nov. from Dolenje Laknice, Slovenia: $\lesssim$ holotype (+ + ).

\begin{tabular}{lccccccc}
\hline & Trochanter & Femur & Patella & Tibia & Metatarsus & Tarsus & Total length \\
\hline Pedipalp & $0.28(0.31)$ & $0.77(0.81)$ & $0.56(0.63)$ & $0.47(0.49)$ & - & $0.30(0.31)$ & $2.38(2.55)$ \\
Leg I & $0.26(0.27)$ & $0.94(0.83)$ & $0.39(0.35)$ & $0.67(0.63)$ & $1.11(1.04)$ & $1.10(0.98)$ & $4.47(4.10)$ \\
Leg II & $0.23(0.23)$ & $1.55(1.40)$ & $0.47(0.50)$ & $0.91(1.16)$ & $2.21(2.24)$ & $1.80(1.61)$ & $7.17(7.14)$ \\
Leg III & $0.22(0.22)$ & $0.82(0.83)$ & $0.36(0.35)$ & $0.63(0.63)$ & $1.22(1.19)$ & $0.97(0.95)$ & $4.22(4.17)$ \\
Leg IV & $0.23(0.23)$ & $1.43(1.35)$ & $0.39(0.41)$ & $0.79(0.91)$ & $1.94(1.90)$ & $1.45(1.23)$ & $6.23(6.03)$ \\
\hline
\end{tabular}

Chelicerae. Ch basal article length 0.65, 2.6 times as long as wide at dorsal hump, with low-arched dorsal margin in front of dorsal indentation, and low ventral round-triangular hump (Fig. 13D). Distal article length 0.67 , max width 0.20 , movable finger length 0.31 .

PediPALPS. Pa-Tr with relatively high, symmetrically, slightly knee-like arched dorsal margin, Pa-Fe with conspicuous distal bonce, Pa-Ti elongated ellipsoid (Fig. 13D). Pa article lengths in Table 8.

Ovipositor. Ovipositor length 0.81 , Rec sem with one tubular and 12 elongated balloon-like vesicles (Fig. 11E).

LeGs. Leg article lengths in Table 8.

\section{Variability}

In eastern Slovenia, males mostly have four thin medio-ventral spines of similar size on the Pa-Fe, the proximal one placed approximately in the middle of the Pa-Fe. In south-eastern Slovenia, some spines can be very small or diminished to a tubercle with a seta. In contrast, in south-western Slovenia, males have three or rarely two large, stout thorns, sometimes resembling those in $N$. b. bidentatum. Further investigation in Croatian Istria is required to elucidate the whole range of morphological variation and the expected transition between the tender eastern and robust western armament of the Pa-Fe.

\section{Remarks}

Nemastoma $b$. gruberi ssp. nov. is the most variable subspecies within $N$. bidentatum. Prior to this study, a few collected individuals had been considered within the variability range of $N$. b. sparsum, e.g., individuals from Buje and Vižinada considered as paratypes of $N$. b. sparsum (NHMW 4700). Accordingly, Novak et al. (2002) and Novak (2004b, 2005b) misidentified specimens, and those from SW Slovenia were erroneously reported (Novak 2005b) as a disjunctive population of $N$. b. sparsum in the Sub Mediterranean region in Slovenia. Near the border of its distribution area in SW Slovenia, $N$. b. gruberi ssp. nov. has relatively uniform, robust Pa-Fe, with 3 subequidistant, rarely 2, large, stout thorns, much different from the armament in the main population.

\section{Distribution}

Croatia, Italy, Slovenia. Vertical distribution in Slovenia: 4-1619 m a.s.l. Type locality: Dolenje Laknice, Mokronog $\left(45.93^{\circ} \mathrm{N}, 15.21^{\circ} \mathrm{E}, 237 \mathrm{~m}\right.$ a.s.1.), Slovenia.

\section{Ecology}

Nemastoma b. gruberi ssp. nov. inhabits various habitat types in lowland and hilly land, from thermophile shrub and Quercetalia pubescenti-petraeae Klika 1928 communities in southwestern regions to riverine, floodplain and submontane woods in southern and south-eastern Slovenia. Phenology: adults eurychronous. 
Nemastoma kozari Novak, Kozel, Podlesnik \& Raspotnig sp. nov. urn:1sid:zoobank.org:act:7F207FF7-9241-4854-A4C3-B7DED507F740

Figs 2, 4F, 5F, 6F, 7F, 8F, 9F, 10F, 11F, 12F, 13E; Table 9

\section{Diagnosis}

Species of the $N . b$. complex with highly arched, symmetrical Pa-Tr dorsal margin, concave dorsal margin of Pa-Ti and equilateral triangular glans, and Rec sem of 5 elongated vesicles attached to a bladder.

\section{Etymology}

The species name 'kozari' is dedicated to Senad Kozar (Travnik), whose friendship and hospitality helped us in a searching expedition on the Vlašić planina Mts.

\section{Material examined}

\section{Holotype}

BOSNIA AND HERZEGOVINA - YK10 • 1 $\delta^{7}$; Mrazišče, the Vlašić planina Mts, Travnik; $44.28^{\circ} \mathrm{E}$, $17.63^{\circ}$ N; 1550 m a.s.1.; 1 Oct. 2019; S. Kozar, J. Podlesnik, P. Kozel and T. Novak leg.; light submontane beech and fir forest litter; PMSL-Opiliones-TN 137/2019.

\section{Paratypes}

BOSNIA AND HERZEGOVINA - YK10 • $2 \widehat{\jmath}, 1$, ; same collection data as for holotype; PMSLOpiliones-TN 137/2019.

\section{Other material}

BOSNIA AND HERZEGOVINA - YK10 • 4 ふぇ, 10 q $q$; Oštrike, Vlašić Planina Mts; 1 Oct. 2019, S. Kozar, J. Podlesnik, P. Kozel and T. Novak leg. (GR5534-5543, GR5547-5550); PMSL.

\section{Description}

\section{Male (holotype)}

BoDy. Length 1.90, body width: 1.41 .

Chelicerae. Ch basal article (without Apo) length 0.61, 2.0 times as long as wide at $\mathrm{Ch}$ max width at dorsal hump. Ch-Apo small, 0.24 times as high as basal article length, rounded-trapezoid, widest distally; Ch-Apo height 0.14 , max width 0.12 , min width 0.09 (at the base), ventral margin slightly concave. Secretion field apically. Distal article length 0.60, max width 0.19, movable finger length 0.23 (Figs 4F, 6F, 9F).

PediPALPS. Pa-Tr relatively very high ( $\mathrm{Pa}-\mathrm{Tr}$ height:length $\sim 0.7: 1), \mathrm{Pa}-\mathrm{Fe}$ moderately robust (Pa-Fe min:max width $\sim 1: 2.7$ ), with 4 subequidistant stout spines in the distal Pa-Fe third. Pa-Pt relatively thin (length:max width $\sim 1: 4.5$ ), Pa-Ti dorsally conspicuously concave (Figs 5F, 6F). Pa article lengths in Table 9.

PenIs. Pe length 1.55, base 0.60, glans 0.13, stylus 0.04 (Figs 7F, 8F).

LeGs. Pseudoarticle leg-Fe formula I-IV: 0-1-1-2. Tarsomere leg formula I-IV: 8-14-8-9. Leg article lengths in Table 9.

\section{Female}

BoDy. Length 2.03, body width 1.67. 
Table 9. Length of appendage-segments in millimeters in $N$. kozari Novak, Kozel, Podlesnik \& Raspotnig sp. nov. from Vlašić planina, Bosnia and Herzegovina: $\widehat{o}$ holotype ( + ).

\begin{tabular}{lccccccc}
\hline & Trochanter & Femur & Patella & Tibia & Metatarsus & Tarsus & Total length \\
\hline Pedipalp & $0.25(0.28)$ & $0.74(0.69)$ & $0.51(0.55)$ & $0.41(0.43)$ & - & $0.28(0.29)$ & $2.19(2.24)$ \\
Leg I & $0.22(0.23)$ & $0.87(0.76)$ & $0.35(0.30)$ & $0.59(0.62)$ & $0.97(0.91)$ & $0.82(0.88)$ & $3.82(3.70)$ \\
Leg II & $0.24(0.24)$ & $1.32(1.22)$ & $0.44(0.36)$ & $1.08(1.00)$ & $1.87(1.72)$ & $1.46(1.37)$ & $6.41(5.91)$ \\
Leg III & $0.23(0.26)$ & $0.82(0.78)$ & $0.33(0.31)$ & $0.53(0.51)$ & $1.09(1.06)$ & $0.82(0.72)$ & $3.82(3.64)$ \\
Leg IV & $0.27(0.26)$ & $1.28(1.23)$ & $0.43(0.38)$ & $0.88(0.77)$ & $1.74(1.69)$ & $1.04(0.79)$ & $5.64(5.12)$ \\
\hline
\end{tabular}

Chelicerae. Ch basal article length 0.63, 2.4 times as long as wide at dorsal hump, with slightly arched dorsal margin in front of indentation, and slightly concave ventral margin (Fig. 13E). Distal article length 0.66 , max width 0.23 , movable finger length 0.28 .

PeDIPALPS. Pa-Tr with moderately high, arched dorsal margin, $\mathrm{Pa}-\mathrm{Fe}$ with conspicuous distal bonce, $\mathrm{Pa}-\mathrm{Pt}$ and Pa-Ti elongated (Fig. 13E). Pa article lengths in Table 9.

Ovipositor. Ovipositor length 0.93 , Rec sem consisted of bladder and 5 elongated sac-like vesicles (Fig. 11F).

LEGS. Leg lengths in Table 9.

\section{Distribution}

Bosnia and Herzegovina, probably endemic to the Vlašić planina Mts. Vertical distribution: $1550-1630 \mathrm{~m}$ a.s.l. Type locality: Mrazišče, Mt. Paljenik in the Vlašić planina Mts, Travnik $\left(44.28^{\circ} \mathrm{N}, 17.63^{\circ} \mathrm{E}, 1550 \mathrm{~m}\right.$ a.s.1.), Bosnia.

\section{Ecology}

Nemastoma kozari sp. nov. individuals have been found in light submontane beech and fir forests, but may inhabit also other vegetation types. Phenology: adults probably eurychronous.

Nemastoma bidentatum martensi Novak, Slana Novak \& Raspotnig ssp. nov. urn:lsid:zoobank.org:act:CCDC54EF-D41A-4547-A4D4-50370212C243

Figs 2-3, 4G, 5G, 6G, 7G, 8G, 9G, 10G, 11G, 12G, 13F; Table 10

\section{Diagnosis}

Subspecies of Nemastoma bidentatum with Ch basal article with a saw-like series of 1-3 $\mu \mathrm{m}$ high denticles on anterior margin of ventral hump, and a row of 5-11 denticles and tubercles in the distal half of Pa-Fe. Rec sem of 12-14 slightly elongated balloon-like vesicles.

\section{Etymology}

The subspecies name 'martensi' is dedicated to Jochen Martens (Mainz), our teacher, colleague and friend, who provided the first modern revision of harvestmen in Slovenia.

\section{Material examined}

\section{Holotype}

SLOVENIA - VL23 • 1 §̊; Poljane pri Podgradu; $45.50^{\circ}$ N, $14.10^{\circ}$ E; 597 m a.s.1.; 25 Sep. 2011; L. Slana Novak and T. Novak leg.; thermophile scrub and mixed forest litter sift; PMSL-Opiliones-TN 287/2011. 


\section{Paratypes}

SLOVENIA-VL23・8 ふぶ, 5 우; same collection data as for holotype; PMSL-Opiliones-TN 287/2011.

\section{Other material}

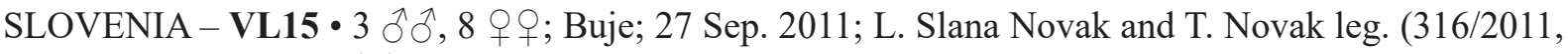
rev. 2015); PMSL・ 2 ô; ibid.; (316a/2011); PMSL・ 1 o ; Mt. Ostrič; 29 Aug. 2014; L. Slana Novak

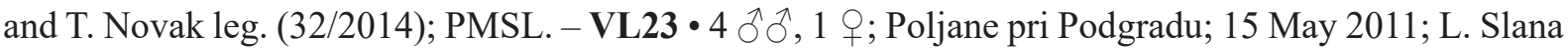

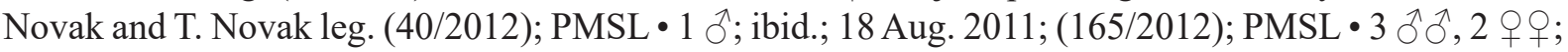
ibid.; 20 May 2012; L. Slana Novak and T. Novak leg. (GR 3597-3601, TN det.); PMSL・ 1 ภ, 2 우; ibid.; 24 Oct. 2013; L. Slana Novak and T. Novak leg. (GR 6017, 6018, 6020, TN det.); PMSL・ 2 $\widehat{\partial}$, 4 우; ibid.; 28 Oct. 2013; L. Slana Novak and T. Novak leg. (GR 4651, 4653, 4654, 4656, 4657, 4659, TN det.); PMSL • 1 §̂, 1 o ; Mt. Velika Medvižica; 18 Jul. 2014; L. Slana Novak and T. Novak leg. (15/2014); PMSL. - VL24 • 1 ठ̧; Mala Plešivica, Golac; 20 Sep. 2012; T. Novak leg. (169/2012); PMSL - 1 đ̧; ibid.; 5 Oct. 2012; L. Slana Novak and T. Novak leg. (PK 20/2012, TN rev. 2018); PMSL • 1 O ; ibid.; 21 May 2014; P. Kozel and T. Novak leg. (PK 18/2014); PMSL • 1 क; ibid.; (PK 18/2014); PMSL - 1 ठ̊; Mt. Mala Pleševica - Mt. Lipica; 19 Oct. 2014; T. Novak leg. (26/2015); PMSL・ 1 ठ̊; Obrov Golac; 19 Sep. 2011; L. Slana Novak and T. Novak leg. (253/2011); PMSL・ 1 §ं; Mt. Velika Pleševica; 19 Oct. 2014; T. Novak leg. (14/2015); PMSL・ 1 ô, 1 क; ibid.; (16/2015); PMSL・ 1 o; Velika vrata, Mt. Velika Pleševica; 6 Oct. 2012; L. Slana Novak and T. Novak leg. (186/2012); PMSL・ 4 ふै ô, 2 우; ibid.; (PK 16/2012, TN rev. 2018); PMSL. - VL25 • 1 +; Buje - Suhorje; 27 Sep. 2011; L. Slana Novak and T. Novak leg. (298/2011); PMSL. - VL26・3 đô;; Fameljska loza, Senožeče; 1 Oct. 2011; L. Slana Novak and T. Novak leg. (351/2011); PMSL. - VL33; PMSL • 3 ふో Novak and T. Novak leg. (269/2011); PMSL. - VL34 • 1 ô, 1 क ; Harije; 19 Sep. 2011; L. Slana Novak and T. Novak leg. (224a/2011); PMSL. - VL43 • 2 q ; Novokrajska jama cave, Cad. No. 810, Novokračine;

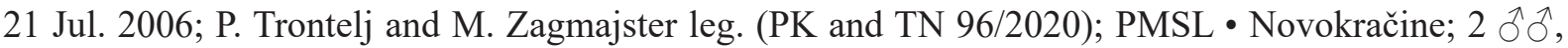
2 우; 27 Sep. 2011; L. Slana Novak and T. Novak leg. (307/2011, rev. 2015); PMSL. - VL44 • 1 ठૈ, 1 +; Jablanica - Trpčane; 9 Sep. 2011; L. Slana Novak and T. Novak leg. (252/2011, rev. 2015); PMSL. -VL53 • 1 đ̂, 1 O; Mt. Šešnovica; 27 Sep. 2011; L. Slana Novak and T. Novak leg. (300/2011); PMSL. -VL54 - 1 9; Bezgovec, Zabiče; 13 Nov.-10 Dec. 2002; B. Drovenik and A. Gergeli leg. (PK 50/2012; TN rev. 2018); PMSL • 1 क; ibid.; 16 Oct.-13 Nov. 2002; B. Drovenik and A. Gergeli leg. (PK 58/2012; TN rev. 2018); PMSL • 1 đ; Mt. Snežnik; 12 Sep. 2018; L. Slana Novak and T. Novak leg. (116b/2018); PMSL. - VL84 - 1 Oૈ, 1 क ; Pleš, Borovec pri Kočevski Reki; 19 Sep. 2013; T. Novak leg. (60/2013, rev. 2015); PMSL. - VL87 • 1 ô; Veliki Grintovec, Zagradec, Žužemberk; 10 May 2008; L. Slana Novak and T. Novak leg. (117/2011, rev. 2015); PMSL.

\section{Description}

Male (holotype)

BoDy. Length 1.63, width 1.26.

Chelicerae. Ch basal article (without Apo) length 0.57, 2.5 times as long as wide at Ch max width at dorsal hump, and with a saw-like series of 1-3 $\mu \mathrm{m}$ high denticles on anterior margin of ventral hump (not visible on Figs). Ch-Apo small, 0.31 times as high as basal article length, rounded-trapezoid, widest distally, highest posteriorly; Ch-Apo height 0.18 , max width 0.15 , min width 0.09 (at the base), frontal margin quarter moon-like. Secretion field medially. Distal article length 0.58 , max width 0.19 , movable finger length 0.23 (Figs 4G, 5G, 6G).

PediPALPS. Pa-Tr oval-trapezoid, moderately high (Pa-Tr height:length $\sim 0.6: 1$ ), Pa-Fe moderately robust (Pa-Fe min:max width 1:3.0), with 7 subequidistant low, stout, irregularly shaped tubercles and denticles in the distal Pa-Fe half. Pa-Pt moderately robust (length: max width $\sim 1: 4.0$ ) (Figs 5G, 6G). Pa article lengths in Table 10. 
Table 10. Length of appendage-segments in millimeters in N. bidentatum martensi Novak, Slana Novak \& Raspotnig ssp. nov. from Poljane pri Podgradu, Slovenia: $\widehat{o}$ holotype (iq).

\begin{tabular}{lccccccc}
\hline & Trochanter & Femur & Patella & Tibia & Metatarsus & Tarsus & Total length \\
\hline Pedipalp & $0.27(0.26)$ & $0.73(0.76)$ & $0.48(0.55)$ & $0.44(0.47)$ & - & $0.27(0.29)$ & $2.19(2.33)$ \\
Leg I & $0.23(0.23)$ & $0.93(0.80)$ & $0.35(0.35)$ & $0.60(0.56)$ & $1.03(0.94)$ & $0.78(0.89)$ & $3.92(3.88)$ \\
Leg II & $0.22(0.23)$ & $1.43(1.35)$ & $0.48(0.45)$ & $1.15(1.25)$ & $1.93(1.80)$ & $1.69(1.65)$ & $6.88(6.63)$ \\
Leg III & $0.24(0.21)$ & $0.83(0.80)$ & $0.33(0.33)$ & $0.60(0.59)$ & $1.09(1.05)$ & $0.83(0.83)$ & $3.93(3.81)$ \\
Leg IV & $0.23(0.21)$ & $1.33(1.30)$ & $0.48(0.38)$ & $0.83(0.83)$ & $1.59(1.63)$ & $0.84(0.83)$ & $5.30(5.18)$ \\
\hline
\end{tabular}

PenIS. Pe length 1.44, base 0.45, glans 0.12, stylus 0.07 (Figs 7G, 8G).

Legs. Pseudoarticle leg-Fe formula I-IV: 0-1-1-2. Tarsomere leg-Fe formula I-IV: 8-17-8-9. Leg article lengths in Table 10.

\section{Female}

BoDY. Length 2.23, width 1.60.

Chelicerae. Ch basal article length 0.60, 2.9 times as long as wide at dorsal hump, with slightly, evenly arched dorsal margin in front dorsal indentation, and medial straight portion in middle of slightly concave ventral margin (Fig. 13F). Distal article length 0.63, max width 0.20, movable finger length 0.23 .

PEDIPALPS. Pa-Tr with moderately high, arched, with straight posterior portion of dorsal margin, $\mathrm{Pa}-\mathrm{Fe}$ slightly widened distally; Pa-Pt, Pa-Ti and Pa-Ta elongated (Fig. 13F). Pa article lengths in Table 10.

OviPositor. Ovipositor length 0.94 , Rec sem of 12-14 slightly elongated vesicles (Fig. 11G).

LEGs. Leg article lengths in Table 10.

\section{Distribution}

Croatia, Slovenia. Vertical distribution in Slovenia: 320-1030 m a.s.1. Type locality: Poljane pri Podgradu $\left(45.50^{\circ} \mathrm{N}, 14.10^{\circ} \mathrm{E}, 597 \mathrm{~m}\right.$ a.s.1.), Slovenia.

\section{Ecology}

Nemastoma b. martensi ssp. nov. is native to lowland to submontane habitats with thermophile scrub and Quercetalia pubescenti-petraeae and Tilia platyphyllos Scop. forest communities, with partly substantial admixture of Fagus sylvatica and Corylus avellana L. in southwestern Slovenia. Phenology: adults probably eurychronous.

Nemastoma bidentatum schmidti Novak, Raspotnig \& Slana Novak ssp. nov. urn:lsid:zoobank.org:act:389E4C27-24B9-44F9-A724-11475CB9E12B Figs 2-3, 4H, 5H, 6H, 7A, H, 8A, H, 9H, 10H, 11H, 12H, 13G; Table 11

Nemastoma triste - Roewer 1917: 149 [partim: Maribor/Marburg], 151 [partim: Postojna/Adelsberg, Divača/Divaca, Ljubljana/Laibach, Zagreb/Agram].

Nemastoma bidentatum - Roewer 1923: 658 [partim: Postojna/Adelsberg, Ljubljana/Laibach, Zagreb/Agram; the other localities refer to N. bidentatum sparsum]. — Hadži 1927: 11-14, figs 81-93; 1928: 9; 1931: 108-109 [partim: Podkoren, Peričnik, Bled]. 
Nemastoma bidentatum bidentatum - Gruber \& Martens 1968: 141, figs 3, 23 [partim: Leutscach]. Marcellino 1973: 194. — Martens 1978: 107, fig 142 [partim: HR: Dugo selo, Zagreb; I: Laghi di Fusine; SLO: Bled, Cerknica, Ljubljana, Mt. Nanos, Postojna]. — Novak 2004a: 218.

Nemastoma quadripunctatum humerale - Hadži 1931: 41, figs 94-98 (by lapsus).

Nemastoma (Lugubrostoma) triste pluridentatum - Hadži 1973a: 43 figs 34a-c [partim: Mt. Šmarna gora, Prestranek].

?Nemastoma bidentatum bidentatum $\times$ N. bidentatum sparsum - Novak \& Gruber 2000: 285 .

\section{Diagnosis}

Subspecies of Nemastoma bidentatum with elongated oval-trapezoid Ch-Apo, sagittally shallowly bilobe apically, wider distally, with a prominent apical pinnacle posteriorly, and conspicuously club-shaped $\mathrm{Pa}$ $\mathrm{Fe}$, with dorsal margin bent in the middle and nearly straight anterior and posterior portions, and evenly arched ventral margin, with 3 stout spines in distal fourth, and low, inconspicuous Pa-Ti hump. Rec sem of one tubular and 12 slightly elongated vesicles.

\section{Etymology}

The subspecies name 'schmidti' is dedicated to Ferdinand Joseph Schmidt (1791-1878; Ljubljana), who collected the first specimens of this taxon preserved in his arthropod collection in the Slovenian Museum of Natural History, Ljubljana.

\section{Material examined}

Holotype

SLOVENIA - VM31 • 1 ð̊; Dolenja Sevnica, Poljane nad Škofjo Loko; $46.12^{\circ}$ N, $14.15^{\circ}$ E; $517 \mathrm{~m}$ a.s.1.; 27 Mar. 2018; T. Novak leg.; mixed forest litter; PMSL-Opiliones-TN 1145/2018.

\section{Paratypes}

SLOVENIA - VM31 • 2 q $\odot$; same collection data as for holotype; PMSL-Opiliones-GR 3809.

\section{Other material}

AUSTRIA - VM74 • 1 đ; Eisenkappel/Železna Kapla - Ebriach/Obirsko, Carinthia; 7 May 2018; T. Novak leg. (38/2018); PMSL • 1 क; Seilach/Sele - Ebriach/Obirsko, Carinthia; 7 May 2018; T. Novak leg. (41/2018); PMSL.

CROATIA - WL67 • 1 đ̃, 1 क; Mt. Medvednica; A. Schönhofer leg. (6/2012, rev. 2019); PMSL.

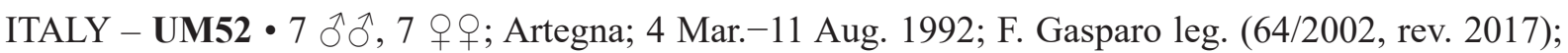
PMSL. - UM62 - 1 đ; A Alta Val Torre; 5 Aug. 1992; G. Governatori leg. (1062/2003, rev. 2017); PMSL

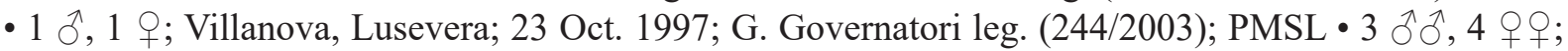
Tarcento/Čenta; 19 Aug. 2001; L. Slana Novak and T. Novak leg. (259/2001, rev. 2017); PMSL 1 ô, 5 우; ibid.; (257/2001, rev. 2018); PMSL • 1 đ̂; Valle del Torre Vedronca valley; 5 Aug. 1992; G.

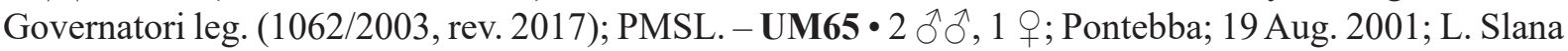
Novak and T. Novak leg. (240/2001, rev. 2017); PMSL. - UM80 • 4 ô;; Altana/Utana; 26 Aug. 2001; L. Slana Novak and T. Novak leg. (283/2001, rev. 2017); PMSL.

SLOVENIA • 1 ð, 1 \& (Schmidt's collection); (84/1985 microscopic preparation, JH det. sub N. triste, TN rev.); PMSL 1 Ô, 1 क (Schmidt's collection); (89/1985 microscopic preparation, JH det. sub N. triste, TN rev.); PMSL. - UL99・ 1 ठ, 2 우우 Panovec; 17 Aug. 2000; T. Novak leg. (668/2000, rev. 2017); PMSL • 1 क; ibid.; 18 Mar. 2001; S. Brelih leg. (63/2001, TN rev. 2019); PMSL・ 9 ठ઼, 5 우; ibid.;

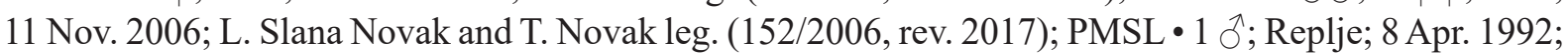
S. Brelih leg. (649/1998, rev. 2017); PMSL. - UM72 • 1 ô; Mt. Kobariški Stol; 24 May 2003; L. Slana 
Novak and T. Novak leg. (720a/2003, TN and SL rev. 2017); PMSL. - UM73 • 1 đ̧; Mt. Kobariški Stol; 8 Jun. 2017; T. Novak leg. (6/2017); PMSL. - UL82 • 1 q; Tavenica, Bela; 10 Oct. 1995; S. Brelih leg. (789/1998, rev. 2018); PMSL • 1 đ̄; Sedlo - Mt. Kobariški Stol; 1 Aug. 1998; L. Slana Novak and T. Novak leg. (52/1999, rev. 2018); PMSL. - UM90 • 2 우; Avče; 2 Aug. 2000; T. Novak leg. (298/2000, rev. 2019); PMSL • 2 $\delta^{\lambda}$; Doblar; 2 Aug. 2000; leg. T. Novak (285/2000, rev. 2017); PMSL • 1 q; ibid.; 17 Jun. 2001; L. Slana Novak and T. Novak leg. (151/2001, rev. 2017); PMSL • 1 ○ं; Lepenka valley, Doblar; 25 Sep. 1998; L. Slana Novak and T. Novak leg. (1795/1998, rev. 2017); PMSL・1 ठ̄; Levpa - Seniški breg; 2 Aug. 2000; T. Novak leg. (319/2000, rev. 2017); PMSL • 1 q; Marija Snežna, Avče; 2 Aug. 2000; T. Novak leg. (293/2000; rev. 2019); PMSL. - UL91 • 1 ठ̊; Kamno; 31 Jul. 1998; L. Slana Novak and T. Novak leg. (1337/1998, rev. 2018); PMSL・ 1 J, 1 क; ibid.; 25 Sep. 1998; L. Slana Novak

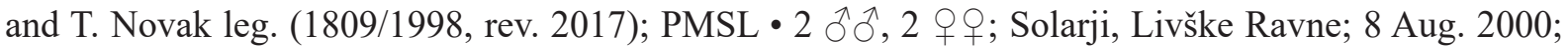
T. Novak leg. (562/2000, TN and SL rev. 2017); PMSL. - UM92 • 6 $\widehat{\jmath}, 1$ q ; Ladrski vrh; 14 May-11 Jun. 2002; B. Drovenik and A. Gergeli leg. (482/2018); PMSL • 1 o; ibid.; 11 Jun.-7 Aug. 2002; B. Drovenik and A. Pirnat leg. (792/2018); PMSL・ 2 ô, 3 우; ibid.; 8 Jul.-7 Aug. 2002; B. Drovenik and A. Pirnat leg. (PK 9/2013, TN rev. 2019); PMSL・ 1 क; ibid.; (PK 10/2013, TN rev. 2019); PMSL・ 1 क; ibid.; (585/2018); PMSL • 1 क; ibid.; 7 Aug. -4 Sep. 2002; B. Drovenik and A. Pirnat leg. (780/2018); PMSL

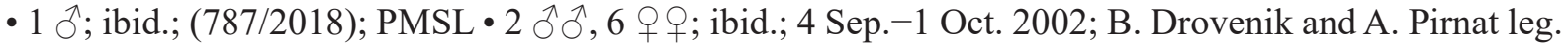

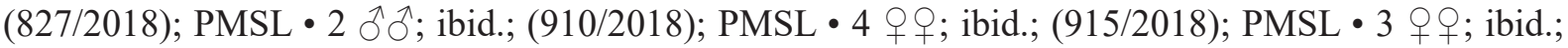

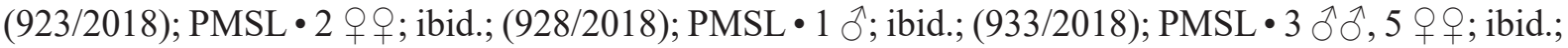
1-29 Oct. 2002; B. Drovenik and A. Pirnat leg. (959/2018); PMSL・ 1 §, 3 우우; ibid.; (961/2018); PMSL • 10 q ; ; ibid.; (965/2018); PMSL • 4 qo , 1 juv.; ibid.; (971/2018); PMSL・ 1 ô, 1 क; ibid.; (974/2018);

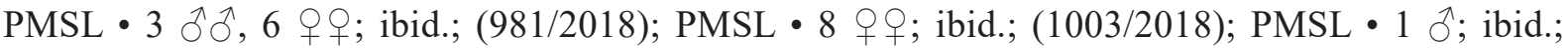
29 Oct. -7 Nov. 2002; B. Drovenik and A. Gergeli leg. (PK 36/2013, TN rev. 2019); PMSL • 3 o ; ; ibid.;

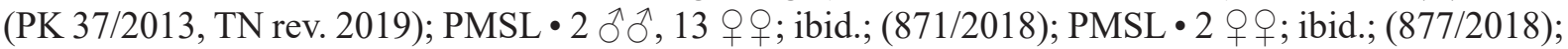

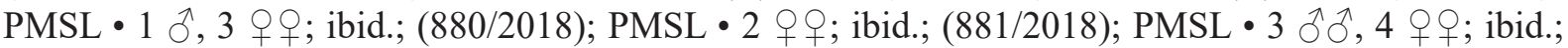

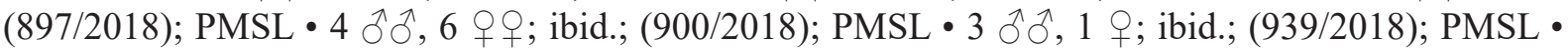
2 우; ibid.; 27 Nov. -21 Dec. 2002; B. Drovenik and A. Gergeli leg. (948/2018); PMSL・ 3 $\widehat{\jmath}, 2$ 우 ibid.; (950/2018); PMSL・ 1 ठ; ibid.; (1010/2018); PMSL・ 3 우; ibid.; (1014/2018); PMSL・ 2 우;

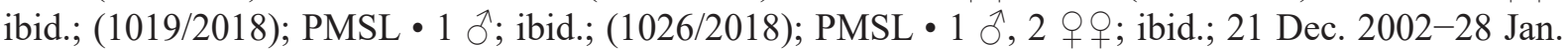

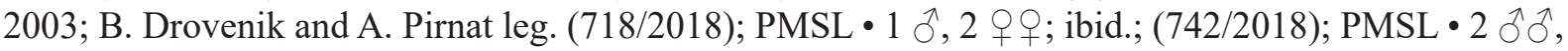

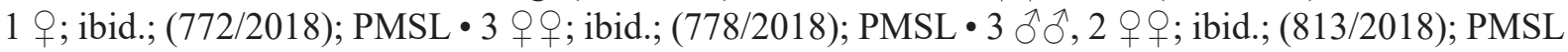

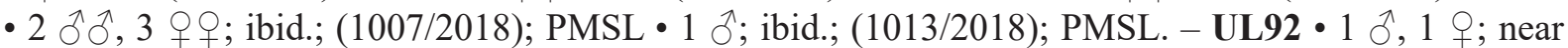
Kamno; Sep.1915; Andreini leg.; PMSL・1 §̂, 2 우; Selce; 10 Oct. 1995; S. Brelih leg. (786/1998, rev. 2017); PMSL・ 1 đ; Šturmi; 8 Aug. 2000; T. Novak leg. (545/2000, rev. 2017); PMSL. - UL93 • 1 क; Bavšica; 9 Aug. 1996; S. Brelih leg. (744/1998, rev. 2018); PMSL • 3 q 9 ; Brvce; 11 Oct. 1995; S. Brelih leg. (745/1998, rev. 2018); PMSL. - VL07 • 1 \%; Velike Žablje; 17 Aug. 2000; T. Novak leg. (677/2000,

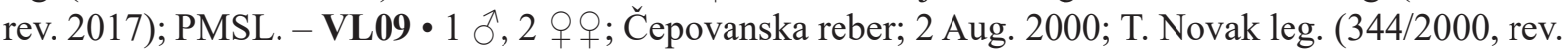
2017); PMSL • 1 万’; Lijak spring, Ozeljan; 17 Apr. 2002; B. Drovenik and A. Gergeli leg. (219/2011, rev. 2017); PMSL • 1 Ȯ; ibid.; 17 Apr.-14 May 2002; B. Drovenik and A. Pirnat leg. (540/2018); PMSL •

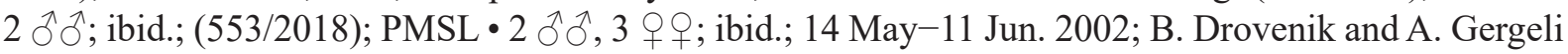
leg. (503/2018); PMSL・ 1 क; ibid.; (518/2018); PMSL・ 1 đ; ibid.; 11 Jun. 2002; B. Drovenik and A. Pirnat leg. (212/2011, rev. 2017); PMSL • 1 ö; ibid.; 8 Jul. 2002; B. Drovenik and A. Pirnat leg. (1093/2018); PMSL・ 1 क; ibid.; (1097/2018); PMSL • 1 q; ibid.; 7 Aug.-4 Sep. 2002; B. Drovenik and A. Gergeli leg. (567/2018); PMSL・ 1 đ̃; ibid.; 1 Oct. 2002; B. Drovenik and A. Pirnat leg. (313/2018); PMSL • 1 o, 1 q; ibid.; 18 Feb. 2003; B. Drovenik and A. Gergeli leg. (200/2011, TN rev. 2017); PMSL - 1 đ̊; Lokve; 2 Aug. 2000; T. Novak leg. (350/2000, rev. 2017); PMSL • 1 q; ibid.; (364/2000, rev. 2017); PMSL • 1 क ; Smrečje, Trnovski gozd; 18 Aug. 1973; I. Sivec leg. (519/1981, JG det. 1982, TN rev. 2018); PMSL. - VL16 • Divača - VL19 • 1 đ; Gorenja Kanomlja; 1 Aug. 2000; T. Novak leg. (401/2000, rev. 2017); PMSL • 1 क; Predmeja; 21 Aug. 2006; L. Slana Novak and T. Novak leg. (4/2006, rev. 2017); PMSL • 2 q ; Srednja Kanomlja; 1 Aug. 2000; T. Novak leg. (391/2000, rev. 2017); PMSL. - VL26• 


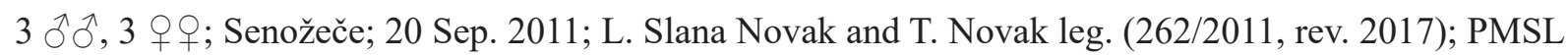

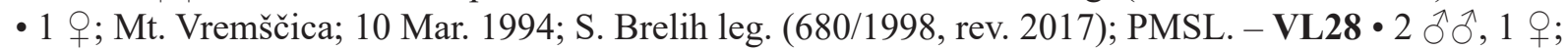
Črni vrh - Col; L. Slana Novak and T. Novak leg. (239/2011, rev. 2017); PMSL. - VL29・ 3 ô ô, 3 우 우; Godovič - Črni vrh; 11 Sep. 2011; L. Slana Novak and T. Novak leg. (241/2011, rev. 2017); PMSL・

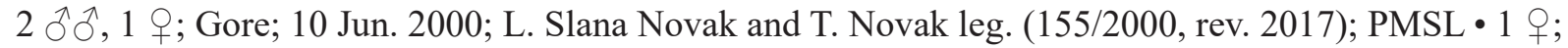
Idrija; Jun. 1997; M. Vončina leg. (1702/1998, rev. 2017); PMSL・ 1 ð̊; ibid.; 10 Jun. 2000; L. Slana Novak and T. Novak leg. (137/2000, rev. 2017); PMSL・ 8 $\widehat{\delta}, 1$ o ; ibid.; (229/2000, TN and SL rev.

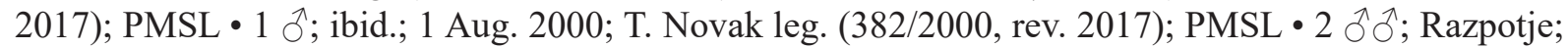
1 Aug. 2000; T. Novak leg. (408/2000, rev. 2017); PMSL • 1 đ̊; Spodnja Idrija; 10 Jun. 2000; L. Slana Novak and T. Novak leg. (143/2000, rev. 2017); PMSL・2 ô ô; Srednja Kanomlja; 1 Aug. 2000; T. Novak leg. (389/2000, rev. 2017); PMSL・ 1 क ; ibid.; (397/2000, rev. 2017); PMSL・1 О̊; Urbanovec; 10 Jun. 2000; L. Slana Novak and T. Novak leg. (149/2000, rev. 2017); PMSL. - VL36・1 đ̊; Jama Sv. Janeza cave, Cad. No. 897, Prestranek; 11 Aug. 1952; E. Pretner leg. (56/1983, JH det., TN rev. 2017); PMSL • 1 P ; ibid.; 29 Aug. 1954; E. Pretner leg. (174/1984, JH det., TN rev. 2018); PMSL・ 1 ○; ibid.; 11 Aug. 1957; E. Pretner leg. (221/1984, TN rev. 2017); PMSL • 3 우; Jernejeva jama cave, Cad. No. 929, Koče; 22 Apr. 2001; S. Vidmar, S. Polak, T. Novak leg. (157/2001, rev. 2008); PMSL・Postojna - VL37・1 万; Belsko; 14 Apr. 2011; L. Slana Novak and T. Novak leg. (86/2011); PMSL・ 1 đ; Landol; 9 May 1994;

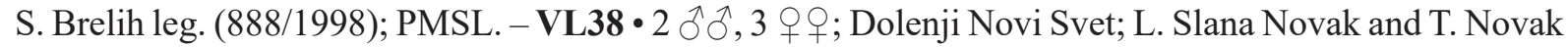
leg. (230/2011); PMSL • 1 q; Novi svet, Hotederšica; 22 Mar. 1993; S. Brelih leg. (855/1998, rev. 2019); PMSL. - VL39 • 3 ภํ, 3 우우 Kugu, Rovte; 11 Sep. 2011; L. Slana Novak and T. Novak leg. (244/2011); PMSL. - VL46• 1 ô, 1 o ; Mt. Javorniki; 9 May 1994; S. Brelih leg. (836/1998); PMSL・ 2 ô ô, 8 우; Mali Kožljek, Mt. Javorniki; 2 Oct. 2011; L. Slana Novak and T. Novak leg. (336/2011); PMSL. - VL47

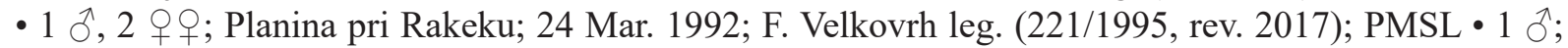
Rakov Škocjan; Jul. 1994; K. Musar leg. (79/1995, rev. 2017); PMSL・ 1 O;; ibid.; 12.-15 May 1994;

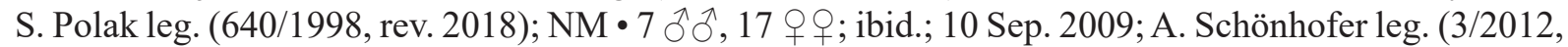
rev. 2017); PMSL. - VL48 • 1 क; Bistra; 29 Mar. 1993; S. Brelih leg. (641/1998, rev. 2017); PMSL • 1 J̊; Laze; 16 Oct.-21 Nov. 1994; M. Kuntner leg. (176/1995, rev. 2017); PMSL・ 1 q; ibid.; Jul.Aug.1994; M. Kuntner leg. (20/1997, rev. 2017); PMSL・ 1 ô, 1 q; ibid.; Aug. 1994; M. Kuntner leg. (183/1995, rev. 2017); PMSL • 1 ô, 1 o ; ibid.; Dec. 1994-Mar. 1995; M. Kuntner leg. (170/1995, rev. 2017); PMSL • 1 क; Vranja jama cave, Cad. No. 88, Laze pri Planini; 7 Jun. 1994; stud. biol. leg. (101/2019); PMSL. - VL49 • 1 q; Retovje, Vrhnika; Jul. 1994, A. Valenčak leg. (17/1997, rev. 2017); PMSL • 1 đ̊; ibid.; Jun. 1998; M. Zagmajster leg. (1493/1998, rev. 2017); PMSL • 1 O; Vrhnika; Jul. 1994; A. Valenčak leg. (17/1997, rev. 2017); PMSL. - VL54 • 1 O̊; Mt. Snežnik; 21 Jul. 2001; B. Bertoncelj, U. Bertoncelj and T. Novak leg. (150/2001, rev. 2017); PMSL・ 1 q; ibid.; 24 Nov. 2003; S. Polak leg. (120/2006, rev. 2018); NM; PMSL. - VL56 • 1 ô, 3 q o ; Cerkniško jezero; 19 Oct. 1994; S. Polak leg.

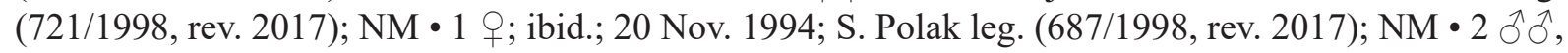
1 क; ibid.; 24 Apr. 2001; S. Brelih leg. (72/2001, rev. 2017); PMSL. - VL57 • 2 $ぇ$, 4 q $\circ$; Dolenje Otave - Selšček; 14 Aug. 2011; L. Slana Novak and T. Novak leg. (36/2011, rev. 2017); PMSL. - VL58 - 1 §; Mt. Krim; 14 Aug. 2011; L. Slana Novak and T. Novak leg. (42/2011, rev. 2017); PMSL • 2 q ;

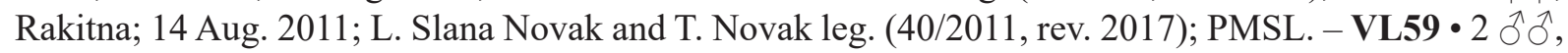
1 क; Bevke; 20 Oct. 2018; L. Slana Novak and T. Novak leg. (136/2018); PMSL・ 1 đ̊; Brezovica pri Ljubljani; 25 May 1997; L. Slana Novak and T. Novak leg. (14/1997, rev. 2018); PMSL・ 1 O; Draga pri Igu; 13 Apr. 1995; S. Brelih leg. (732/1998, rev. 2018); PMSL・ 3 ổ 0 , 10 q ; Z Žabnica; 14 Aug. 2011; L. Slana Novak and T. Novak leg. (33/2011, rev. 2017); PMSL. - VL68 - 1 đ, 3 q ; ; Iška vas - Mt. Krim; 14 Aug. 2011; L. Slana Novak and T. Novak leg. (48/2011, rev. 2017); PMSL・ 1 đ̇; ibid.; 31 Mar. 1997; S. Brelih leg. (53/2001, rev. 2017); PMSL • 1 क; Kremenica, Ig; 29 Jun. 1980 (1155/1981, JG det., TN rev. 2018); PMSL • 1 क; ibid.; 2 Mar. 1996; S. Brelih leg. (45/2001, rev. 2017); PMSL • 1 ठ; ibid.; 1 May 1997; S. Brelih leg. (101/2001, rev. 2017); PMSL・ 1 đ̇; ibid.; 7 Jun. 1997; S. Brelih leg. (117/2001,

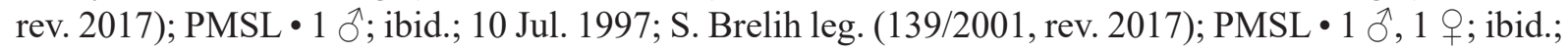
25 Aug. 1997; S. Brelih leg. (113/2001, rev. 2017); PMSL• 1 क; Mt. Mokrec; 18 Jul. 1980 (LSN 151/1984, 
TN rev. 2017); PMSL・ 1 P; Ščurki, Turjak; 3 May 2008; L. Slana Novak and T. Novak leg. (155/2011, rev. 2017); PMSL • 1 क; ibid.; 20 Jun. 1980; (1149/1981, JG det., TN rev. 2018); PMSL・ 1 क; Škrilje; 20 Jun. 1980; (1159/1981, JG det., TN rev. 2018); PMSL. - VL69 • 2 우; Šmarje Sap; 10 May 2008; L. Slana Novak and T. Novak leg. (119a/2011, rev. 2017); PMSL・ 1 đ, 1 q; ibid.; (147/2011, rev. 2018); PMSL. - VL79 • 1 ภ, 4 우; Brinjski hrib; L. Slana Novak and T. Novak leg. (99/2012, TN and SL rev. 2017); PMSL • 1 ơ; Stara vas; 16 Mar. 1993; S. Brelih leg. (665/1998, rev. 2017); PMSL. - VL85 • 1 क ; Mt. Stojna; 21 Oct. 1996; S. Brelih leg. (90/2001, rev. 2017); PMSL. - VL89 • 1 o, 4 o $q$; Vintarjevec; T. Novak leg. (173/2011, rev. 2017); PMSL・ 2 $\widehat{\jmath}, 4$ q ; ; ibid.; (178/2011, rev. 2017); PMSL. - VL93 - 1 q; Kostel, Dolenja Žaga; 16 Apr. 1996; S. Brelih leg. (737/1998, rev. 2018); PMSL. - VL99 • 1 क; Cerovec, Gabrovka; L. Slana Novak and T. Novak leg. (74/2007, rev. 2017); PMSL. - VM00 • 1 ð̄, 2 우; Cvetrež, Voglarji; 2 Aug. 2000; T. Novak leg. (324/2000, TN and SL rev. 2017); PMSL・ 5 ðో 4 우; Čepovan; 1 Aug. 2000; T. Novak leg. (361/2000, rev. 2017); PMSL・1 Ȯ; Čepovan - Most na Soči; 10 Jun. 1997; S. Brelih leg. (110/2001, rev. 2017); PMSL・1 O̊; Preval, Lokovec, Čepovan; 2 Aug. 2000; T. Novak leg. (329/2000, rev. 2017); PMSL. - VM05 • several specs; Podkoren; J. Hadži leg.; PMSL. - VM09 • 1 9; Smrečje; 18 Aug. 1973; I. Sivec leg. (519/1981, JG det., TN rev. 2018); PMSL.

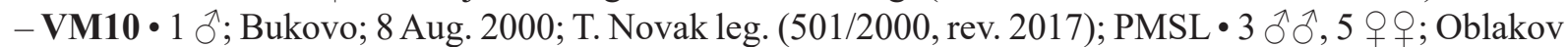
vrh; 1 Aug. 2000; T. Novak leg. (377/2000, rev. 2017); PMSL・ 1 ○; Stopnik; 8 Aug. 2000; T. Novak leg. (492/2000, rev. 2018); PMSL. - VM12 • 1 क; Šavnica, Bohinjska Bistrica; 26 Jul. 2002; B. Drovenik and A. Pirnat leg. (1094/2018); PMSL. - VM13 - 1 क; Suha; 25 Oct. 1993; S. Brelih leg. (868/1998, rev. 2018); PMSL. - VM14 • 1 +; Peričnik waterfall, Vrata valley; 14 Aug. 1928; J. Hadži leg.; PMSL. VM20 • 1 क; Fužine; 8 Aug. 2000; T. Novak leg. (484/2000, rev. 2017); PMSL • 1 ô, 1 q; Govejk; 8 Aug. 2000; T. Novak leg. (486/2000, rev. 2017); PMSL • 1 + ; Osojnica; 8 Aug. 2000; T. Novak leg. (498/2000,

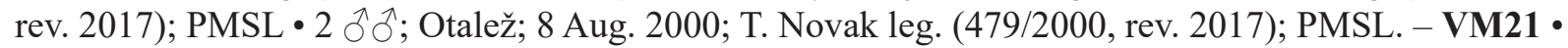

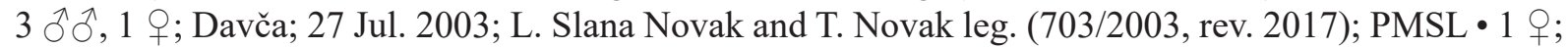
ibid.; (706/2003, rev. 2017); PMSL・ 1 ð’; Velbnik, Mt. Porezen; 7 Jul. 2003; L. Slana Novak and T. Novak leg. (700/2003); PMSL. - VM22 • 1 q; Nomenj; 25 Oct. 1993; S. Brelih leg. (849/1998, rev. 2018); PMSL. - VM23 - 2 q ; t the Pokljuka Mts; 19 Jul. 1940; J. Hadži leg. (323/1983, rev. 2017); PMSL. VM30 • 1 ô, 1 P; Gorenja vas - Lučine; 15 May 1994; S. Brelih leg. (862/1998, rev. 2017); PMSL •

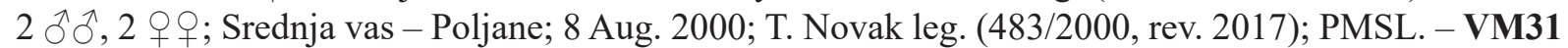

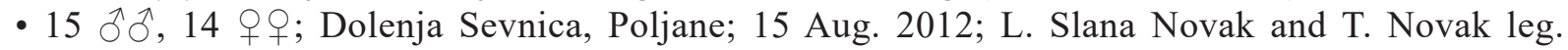
(GR 3777-3783, 3805-3811, 3819-3825, 3833-3835); PMSL. - VM32 • 1 ㅅ, 2 우우 Selca; 15 Aug. 2012; L. Slana Novak and T. Novak leg. (139/2012, TN and SL rev. 2017); PMSL. - VM33 • 1 万; Begunje; 12 Aug. 1985; L. Slana Novak, M. Slana Novak and T. Novak leg. (166/1997, TN and SL rev.

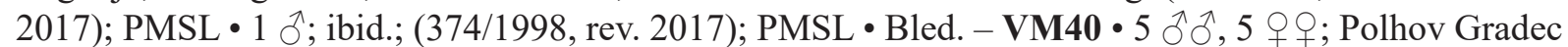
- Črni vrh; 11 Sep. 2011; L. Slana Novak and T. Novak leg. (235/2011, rev. 2017); PMSL. - VM41 •

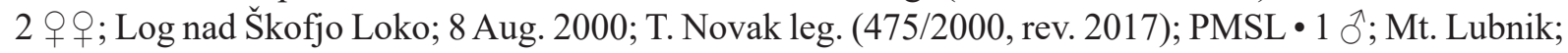

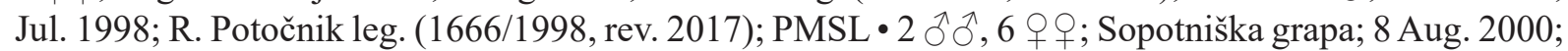

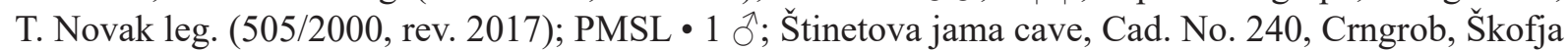
Loka; 25 Jul. 1980; T. Novak, I. Sivec leg. (LSN 214/1984, TN rev. 2019); PMSL. - VM42 • 1 O;; Bukovščica; 15 May 1994; S. Brelih leg. (881/1998, rev. 2017); PMSL・ 1 ơ; Mt. Šmarjetna gora; 27 Apr.-4 May 1987; A. Ziherl, T. Trilar leg. (492/1998, rev. 2017); PMSL • 1 +; ibid.; 18-24 Oct. 1987; A. Ziherl, T. Trilar leg. (483/1998, rev. 2017); PMSL • 1 ô; ibid.; (513/1998, TN and SL rev. 2017); PMSL. - VM43 • 1 O ; Kriška planina; May 1968; (1218/1981, JG det., TN rev. 2017); PMSL • 1 o; Križe, Tržič; 6 Aug. 2015; L. Slana Novak and T. Novak leg. (101/2015, rev. 2017); PMSL. - VM50 • 1 q; Ljubljana; Nov. 1986; (631/2002, rev. 2017); PMSL • 1 q; ibid.; Nov. 1987; (125/1997, TN and

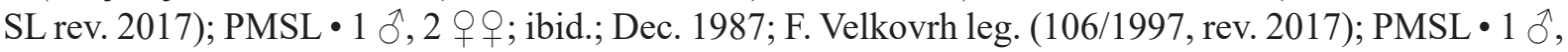
1 q; ibid.; Mar. 1990; F. Velkovrh leg. (285/1995, rev. 2017); PMSL・ 1 \%; Podutik; Jun. 1998; (1648/1998, rev. 2017); PMSL • 1 q; ibid.; Oct. 1928; J. Staudacher leg. (16/1983 microscopic preparation, rev. 2017); PMSL • 1 क ; ibid.; May-Jun. 2000; A. Pokrivač leg. (PK and TN 74/2020); PMSL • 1 क ; Preval, Ljubljana; 18 Mar. 1993; S. Brelih leg. (664/1998, rev. 2017); PMSL. - VM51 • 1 ô;; Mt. Grmada, Medno; 28 Jul. 


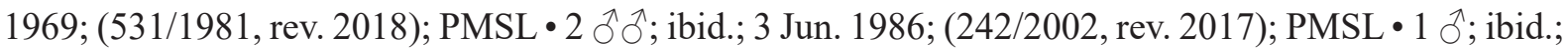
28 Jul. 1969; (531/1981, rev. 2018); PMSL • 1 ô; ibid.; 15 May 1970; (526/1981, rev. 2018); PMSL・ 1 q; ibid.; 19 Apr.-15 May 2002; B. Drovenik and A. Gergeli leg. (188/2011, rev. 2017); PMSL • 1 क; ibid.; (446/2018); PMSL • 1 +; ibid.; 15 May 2002; B. Drovenik and A. Pirnat leg. (216/2011, rev. 2017);

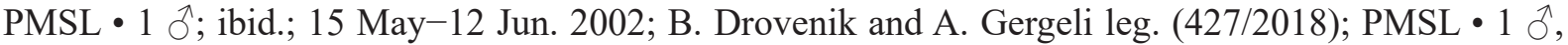
1 q; ibid.; (523/2018); PMSL・ 1 ô; ibid.; (558/2018); PMSL・ 1 q; ibid.; 12 Jun.-9 Jul. 2002; B. Drovenik

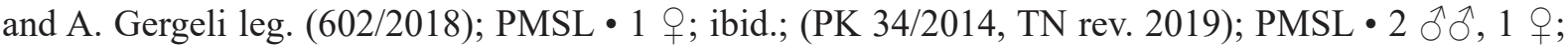
ibid.; 8 Aug.-6 Sep. 2002; B. Drovenik and A. Gergeli leg. (PK 86/2012; TN rev. 2019); PMSL 1 ठ̊; ibid.; 6 Sep. 2002; B. Drovenik and A. Pirnat leg. (354/2018); PMSL • 1 \&; ibid.; 2 Oct. 2002 (173/2003, rev. 2017); PMSL・ 1 क ; ibid.; (1100/2018); PMSL • 2 q 9 ; ibid.; (1124/2018); PMSL・ 1 क; ibid.; 28 Oct. -25 Nov. 2002; B. Drovenik and A. Gergeli leg. (PK 28/2012, TN rev. 2019); PMSL・ 1 ठ; ibid.; (264/2018); PMSL • 1 ; ibid.; 25 Nov. 2002; B. Drovenik and A. Pirnat leg. (277/2018); PMSL 4 우; ibid.; (279/2018); PMSL・ 1 o; ibid.; 19 Mar. 2003; B. Drovenik and A. Pirnat leg. (330/2018); PMSL • 1 9; ibid.; (375/2018); PMSL・ 3 đ̃ ô, 2 우; Mavčiče; Jun. 1998; A. Žunič leg. (1568/1998, rev. 2017);

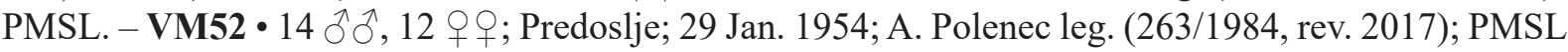

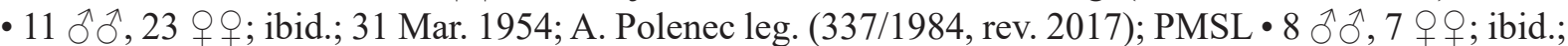
10 Oct. 1954; A. Polenec leg. (106/1984, rev. 2017); PMSL・ 3 ổ, 2 q $q$; ibid.; 15 Oct. 1954; A. Polenec leg. (351/1984, TN and SL rev. 2017); PMSL 6 ô $\hat{o}, 14$ 우; ibid.; 24 Oct. 1954, A. Polenec leg. (63/1984, rev. 2017); PMSL・ 4 ô $\widehat{\jmath}, 7$ 우; ibid.; 30 Oct. 1954; A. Polenec leg. (416/1984, TN and SL

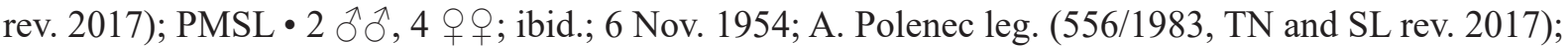

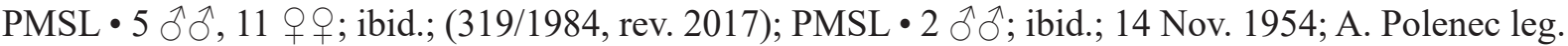

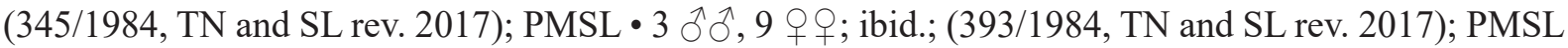

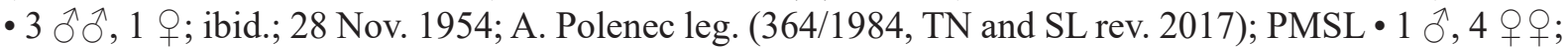

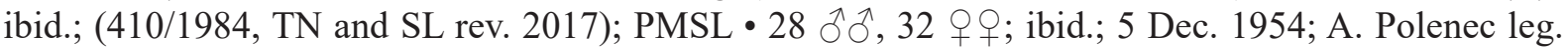
(382/1984, TN and SL rev. 2017); PMSL • 1 \%; ibid.; 18 Dec. 1954; A. Polenec leg. (20/1984, rev. 2017); PMSL・ 32 ๙ึ, 34 우; ibid.; (58/1984, rev. 2017); PMSL・ 1 q; ibid.; 31 Dec. 1954; A. Polenec leg.

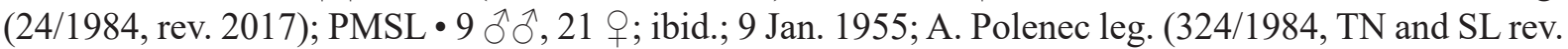

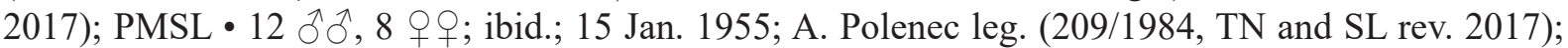

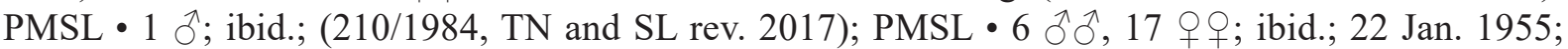
A. Polenec leg. (342/1984, TN and SL rev. 2017); PMSL・ 18 $\widehat{\partial}$, 15 9 우; ibid.; 7 Feb. 1955; A. Polenec leg. (103/1984, TN and SL rev. 2017); PMSL • 20 ภ, 27 우; ibid.; 12 Feb. 1955; A. Polenec leg. (242/1984, TN and SL rev. 2017); PMSL • 1 9; ibid.; 15 Feb. 1955; A. Polenec leg. (328/1984, TN and SL rev. 2017); PMSL • 7 đ̊ 2017); PMSL • 2 + O; ibid.; 25 Mar. 1955; A. Polenec leg. (29/1984, TN and SL rev. 2017); PMSL・1 O;;

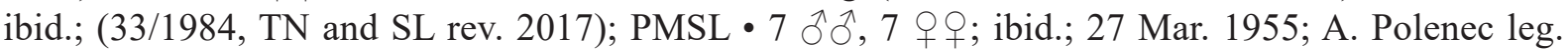

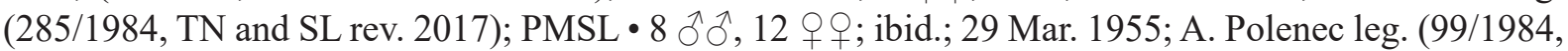

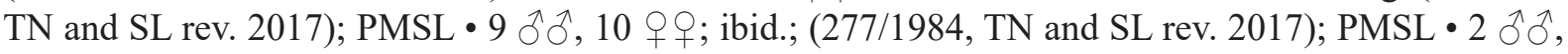

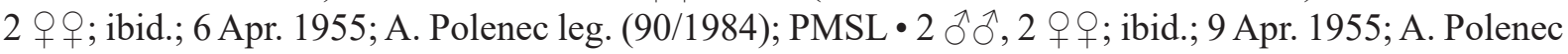
leg. (229/1984, TN and SL rev. 2017); PMSL・ 1 ô, 1 ○; ibid.; 8 May 1955; A. Polenec leg. (69/1984,

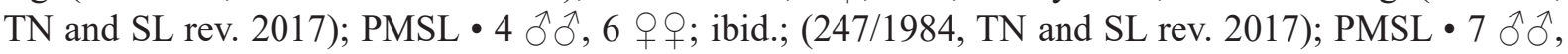

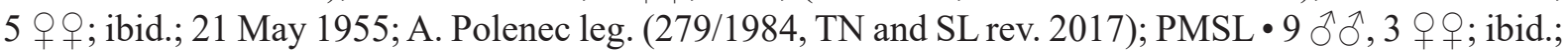
4 Jun. 1955; A. Polenec leg. (298/1984, TN and SL rev. 2017); PMSL・ 1 q; ibid.; (333/1984, TN and SL rev. 2017); PMSL • 2 o+ ; ibid.; 23 Apr. 1955; A. Polenec leg. (313/1984, TN and SL rev. 2017);

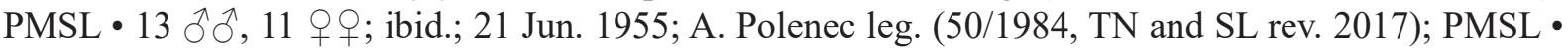
3 우; ibid.; 2 Jul. 1955 (379/1984, TN and SL rev. 2017); PMSL • 1 q; ibid.; 27 Aug. 1955; A. Polenec leg. (253/1984, TN and SL rev. 2017); PMSL • 1 क; ibid.; (305/1984, TN and SL rev. 2017); PMSL •

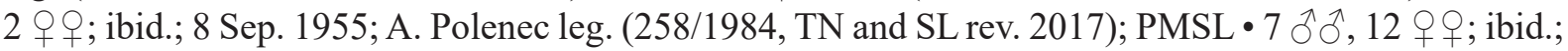
8 Oct. 1955; A. Polenec leg. (362/1983, TN and SL rev. 2017); PMSL・ 1 ô, 2 o o+ ; ibid.; 22 Oct. 1955; A. Polenec leg. (383/1983, TN and SL rev. 2017); PMSL・ 3 ô ô, 4 우; ibid.; (389/1983, rev. 2017);

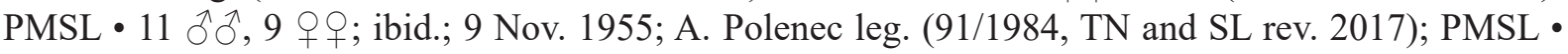


$2 \widehat{\jmath} \widehat{\partial}, 3$ 우; ibid.; 16 Apr. 1956; A. Polenec leg. (482/1983, TN and SL rev. 2017); PMSL・ 1 ภ, 2 우; ibid.; 22 Apr. 1956; A. Polenec leg. (280/1983, TN and SL rev. 2017); PMSL • 1 +; ibid.; 26 May 1957; A. Polenec leg. (441/1983, TN and SL rev. 2017); PMSL • 1 đ; ibid.; 4 Jun. 1956; A. Polenec leg. (289/1984, TN and SL rev. 2017); PMSL • 1 क; ibid.; 22 Jun. 1956; A. Polenec leg. (472/1983); PMSL - 1 ठ̊; ibid.; 8 Jul. 1956; A. Polenec leg. (208/1983, TN and SL rev. 2017); PMSL • 1 ô; ibid.; 22 Jul. 1956; A. Polenec leg. (LSN 9/1983, TN rev. 2018); PMSL・ 1 ó; ibid.; (532/1983, TN and SL rev. 2017);

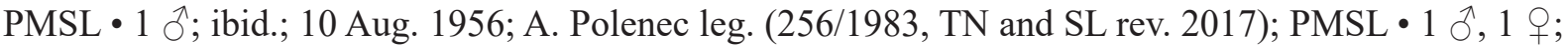
ibid.; 24 Oct. 1956; A. Polenec leg. (537/1983, TN and SL rev. 2017); PMSL • 5 ふึَ, 11 q ; ibid.; 29 Oct. 1956; A. Polenec leg. (508/1983, TN and SL rev. 2017); PMSL・ 5 ô ô, 1 O ; ibid.; 30 Nov. 1956; A. Polenec leg. (423/1983, TN and SL rev. 2017); PMSL・ 1 O , 5 q o ; ibid.; 16 Dec. 1956; A. Polenec

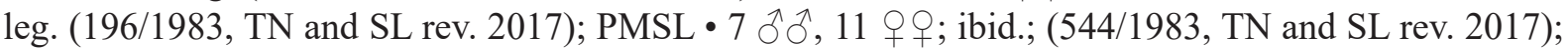
PMSL • 2 ๙ 4 우; ibid.; (295/1984, TN and SL rev. 2017); PMSL・ 8 $\widehat{\jmath}, 10$ 오; ibid.; 20 Feb. 1957; A. Polenec

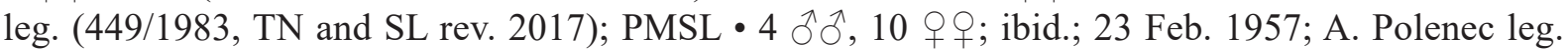

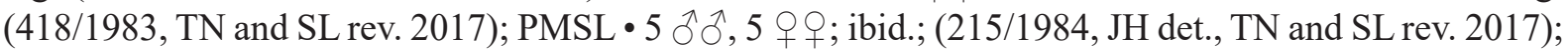

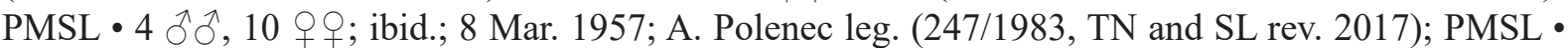

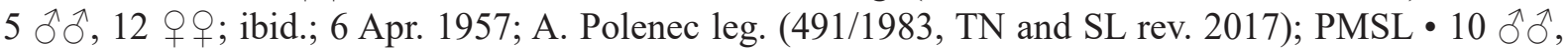

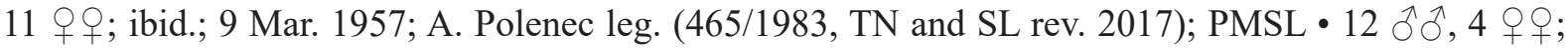
ibid.; 3 May 1957; A. Polenec leg. (506/1983, TN and SL rev. 2017); PMSL・ 5 $\widehat{\jmath}, 5$ $q$ $q$; ibid.; 18 May 1957; A. Polenec leg. (551/1983, TN and SL rev. 2017); PMSL. - VM60 - 12 ô Jun. 1972; (518/1981, JG det., TN rev. 2020); PMSL • 1 ô; ibid.; 26 May 1986; Hribernik, Koren and Mencinger leg. (255/2002, rev. 2017); PMSL. - VM61 - 4 ô, 1 o ; Mt. Gobavica, Mengeš; 22 Aug. 2011; L. Slana Novak and T. Novak leg. (97/2011, rev. 2017); PMSL • 1 q; Rašica; Jun. 1994; H. Urbas leg. (241/1995, TN rev. 2017); PMSL. - VM62 • 1 §, 1 q; Stahovica - Ambrož; 17 Aug. 2011; L. Slana Novak and T. Novak leg. (61/2011, rev. 2017); PMSL. - VM63 • 1 o; Mt. Kalški greben; 12 Aug. 2009; L. Slana Novak and T. Novak leg. (28/2009, rev. 2017); PMSL・ 1 juv.; ibid.; (31/2009, rev. 2017); PMSL

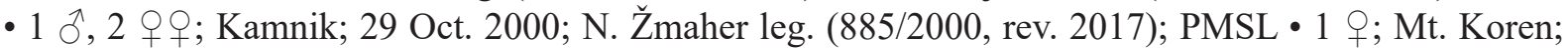
8 Sep. 2009; L. Slana Novak and T. Novak leg. (50/2009, rev. 2017); PMSL・1 đ̇; Mt. Košutna; 22 Sep. 2009; L. Slana Novak and T. Novak leg. (68/2009, rev. 2017); PMSL・1 9 ; Mt. Mokrica; 22 Sep. 2009; L. Slana Novak and T. Novak leg. (80/2009, rev. 2017); PMSL. - VM70 • 1 $\hat{\jmath}, 3$ q $q$; Jevnica; 8 Jul. 1986; L. Slana Novak and T. Novak leg. (538/2002, rev. 2017); PMSL・ 5 $\hat{\jmath} \widehat{\partial}, 3$ 우 ; Senožeti - Velika vas; 17 Aug. 2011; L. Slana Novak and T. Novak leg. (55/2011, rev. 2017); PMSL・ 2 $ð$, 3 q $\odot$; Spodnja Javoršica; 16 Aug. 2000; T. Novak leg. (603/2000, rev. 2017); PMSL・1 ${ }^{\top}$; Vinje; 16 Aug. 2000; T. Novak leg. (693/2000, rev. 2017); PMSL. - VM71 • 1 đ; Brezje pri Dobu; 16 Aug. 2000; T. Novak leg. (605/2000,

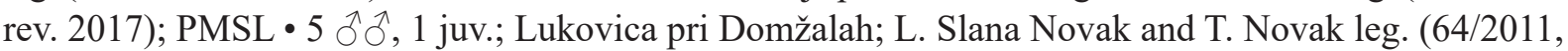
rev. 2017); PMSL • 1 क ; Prevoje pri Šentvidu, Šentvid pri Lukovici; 30 Mar. 1993; S. Brelih leg. (637/1998,

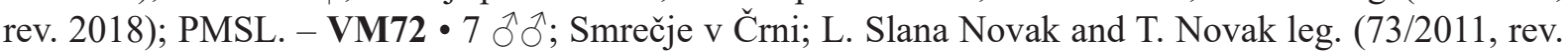
2017); PMSL. - VM73 • 1 ô, 1 क ; Robanov kot; 4 Aug. 2015; T. Novak leg. (85/2015, rev. 2017); PMSL.

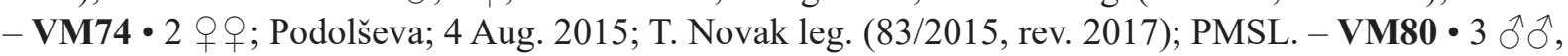
3 우; Kresnice - Litija; 9 Jul. 1986; L. Slana Novak and T. Novak leg. (496/2002, rev. 2017); PMSL•

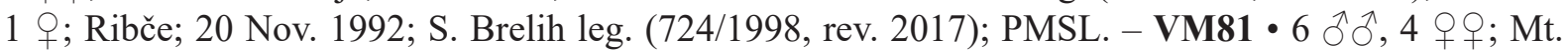
Limbarska gora; 17 Aug. 2011; L. Slana Novak and T. Novak leg. (68/2011, rev. 2017); PMSL • 1 क; Negastern; 16 Aug. 2000; T. Novak leg. (593/2000, rev. 2017); PMSL・ 1 क; Podmilj; 10 May 2008; L. Slana Novak and T. Novak leg. (106/2012, rev. 2017); PMSL. - VM82 • 3 $\widehat{\jmath}, 1$, ; Mt. Gradišče, Bočna, Gornji Grad; 17 Aug. 2011; L. Slana Novak and T. Novak leg. (75/2011, rev. 2017); PMSL. VM83 • 1 ठ̊; Primož pri Ljubnem; 31 Mar. 1994; S. Brelih leg. (875/1998, rev. 2017); PMSL. - VM84 - 1 o; Bistra; 4 Aug. 2015; T. Novak leg. (89/2015, rev. 2017); PMSL. - VM85 • 1 ô, 1 q; Najbrževo, Mt. Peca; 10 Aug. 1999; F. Janžekovič leg. (82/2002, JG det., TN rev. 2018); PMSL・ 1 ô; ibid.; 21 Oct. 1999; F. Janžekovič leg. (45/2003, rev. 2018); PMSL・ 1 đ, 2 क o ; Topla valley; 12 Jul. 2003; L. Slana Novak and T. Novak leg. (187/2003, rev. 2017); PMSL. - VM90 • 1 \&; Cerovec, Gabrovka; 15 Aug. 
NOVAK T. et al., Nemastoma bidentatum (Opiliones: Nemastomatidae) revison

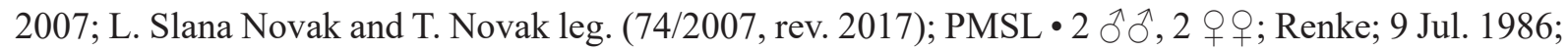

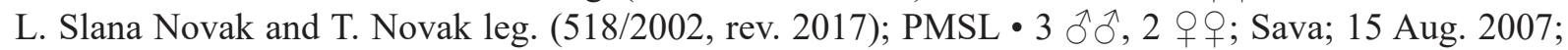
L. Slana Novak and T. Novak leg. (55/2007, rev. 2017); PMSL. - VM91 • 1 Oे, 4 q O ; Mt. Čemšenik;

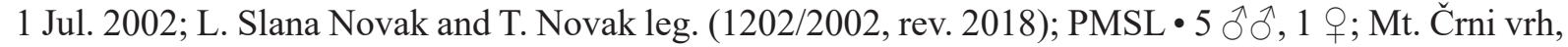
Mt. Čemšeniška planina; 21 Jul. 2002; L. Slana Novak and T. Novak leg. (1171/2002, rev. 2018); PMSL - 1 क; Sv. Primož in Felicijan, Mt. Čemšeniška planina; 21 Jul. 2002; L. Slana Novak and T. Novak leg. (1197/2002, rev. 2017); PMSL. - VM93 - 1 đo, 1 o+; Mt. Golte; 14 Dec. 1984; A. Polenec leg. (LSN 57/1985, TN rev. 2018); PMSL・1 Ȯ; Žlabor; 31 Mar. 1994; S. Brelih leg. (886/1998, rev. 2017); PMSL. -VM94・ 1 ふै, 1 q; Florjan; 6 Jul. 2003; L. Slana Novak and T. Novak leg. (196/2003, rev. 2018); PMSL • 1 ठં; Mt. Smrekovec; 18 Sep. 2000; (579/2002, rev. 2017); PMSL. - VM95 • 3 ðð; Tolsti vrh, Ravne

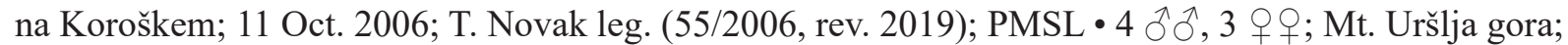
L. Slana Novak and T. Novak leg. (65/2006, TN and SL rev. 2017); PMSL. - VM96 • 2 $ð$, 3 우; Jamnica; 10 Oct. 2006; T. Novak leg. (11/2006, rev. 2019); PMSL・ 1 Ô, 1 q; Zgornja Jamnica; Ravne na Koroškem; 10 Oct. 2006; T. Novak leg. (13/2006, rev. 2018); PMSL. - WL07 • 1 đ; Mali Lipovec; 10 May 2008; L. Slana Novak and T. Novak leg. (107/2011, rev. 2017); PMSL. - WL19 • 1 đ̊; Krmelj - Tržišče; 15 Aug. 2007; L. Slana Novak and T. Novak leg. (66/2007, rev. 2017); PMSL. - WL39 • 1 đ̃; Arto; 18 Nov. 1994; S. Brelih leg. (188/2001, rev. 2017); PMSL. - WL48 • 1 o, 4 q $ᄋ$; Prilipe, Brežice; 26 May 1984; L. Slana Novak and T. Novak leg. (LSN 228/1984, TN rev. 2018); PMSL. - WM00 • 1 क; Sopota valley, Podkum; 1 Apr. -6 May 2002; B. Drovenik and A. Pirnat leg. (642/2018); PMSL • 1 क; ibid.; (648/2018); PMSL • 1 ठ̋; ibid.; 27 May 2002; B. Drovenik and A. Pirnat leg. (214/2011, ver. 2017); PMSL 1 đo; ibid.; 27 May-1 Jul. 2002; B. Drovenik and A. Gergeli leg. (185/2011, rev. 2017); PMSL - 3 우; ibid.; (187/2011, rev. 2017); PMSL • 1 क; ibid.; (189/2011, rev. 2017); PMSL • 2 q ; ibid.; 20 Aug.-19 Sep. 2002; B. Drovenik and A. Gergeli leg. (PK 24/2012, TN rev. 2018); PMSL • 1 q; ibid.;

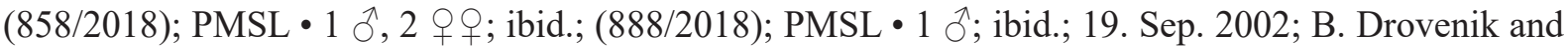
A. Pirnat leg. (256/2018); PMSL 1 क; ibid.; 19 Sep.-15 Oct. 2002; B. Drovenik and A. Pirnat leg. (943/2018); PMSL • 1 O; ibid.; (985/2018); PMSL • 1 q; ibid.; 15 Oct. 2002; B. Drovenik and A. Pirnat leg. (1030/2018); PMSL・ 1 क; ibid.; (1078/2018); PMSL・ 2 o of ; ibid.; 15 Oct. 2002-12 Nov. 2002; B. Drovenik and A. Gergeli leg. (PK 81/2012, TN rev. 2018); PMSL • 1 ○; ibid.; (898/2018); PMSL・

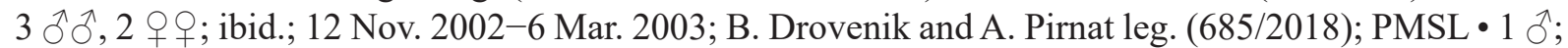
ibid.; (713/2018); PMSL・ 1 q, 1 juv.; ibid.; (736/2018); PMSL・ 1 ô, 3 q 9 ; ibid.; (743/2018); PMSL・ 1 juv.; ibid.; 6 Mar. 2003; B. Drovenik and A. Pirnat leg. (212/2018); PMSL • 1 9; ibid.; (238/2018); PMSL・ 1 ơ; Šklendrovec, Mt. Kum; 22 Apr. 1992; S. Brelih leg. (727/1998, rev. 2017); PMSL. - WM02 - 3 đ̂̃, 1 क; Braslovško jezero, Braslovče; 26 Oct. 2013; T. Novak leg. (35/2013, rev. 2017); PMSL.

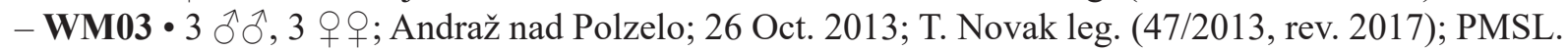

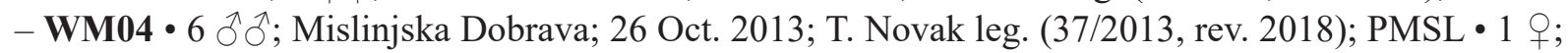
Rdečka jama cave, Cad. No. 3488, Mt. Rdečki vrh; 10 Jul. 1977; V. Kuštor, T. Novak leg. (1119/1981, JG det., TN rev. 2018); PMSL. - WM06 • 1 ô, 1 q; Dom na Košenjaku; 14 Oct. 1995; S. Hudrap and M. Pavlin leg. (LSN 103/1996, TN rev. 2004); PMSL・ 1 §, 1 q; Mt. Košenjak; 14 Oct. 1995; S. Hudrap

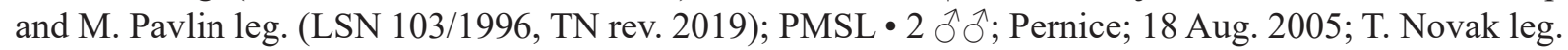
(57/2005, rev. 2018); PMSL・ 1 đ; ibid.; 18 Aug. 2005; T. Novak leg. (66a/2005, rev. 2017); PMSL・

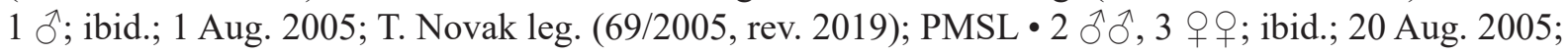
L. Slana Novak and T. Novak leg. (86/2005, rev. 2017); PMSL・ 1 ô; ibid.; 22 Aug. 2005; L. Slana Novak and T. Novak leg. (108/2005, rev. 2017); PMSL • 1 ô; ibid.; (110/2005, rev. 2017); PMSL・ 1 क; ibid.; (112/2005, rev. 2017); PMSL • 2 ôे, 1 q; ibid.; (114/2005, rev. 2018); PMSL. - WM13 • $1 \hat{\jmath}, 1$ क;

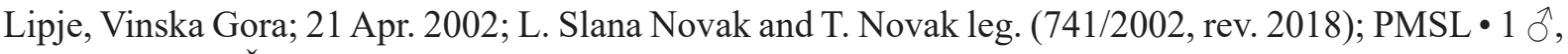
1 O; Vodosteč, Črnova; 21 Apr. 2002; L. Slana Novak and T. Novak leg. (737/2002, rev. 2019); PMSL - 1 Oे, 1 क; Zavrh nad Dobrno; 22 Apr. 1995; S. Brelih leg. (770/1998, rev. 2018); PMSL. - WM14 •

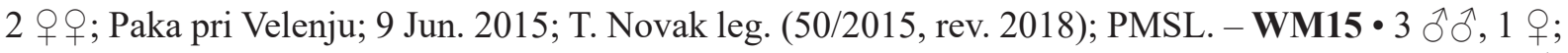

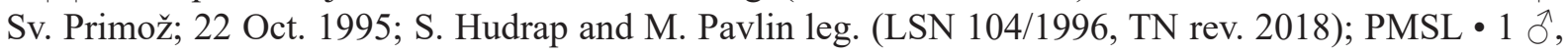
1 ; Mt. Kremžarjev vrh; 26 May 2005; L. Slana Novak and T. Novak leg. (187/2005, rev. 2017); PMSL. 


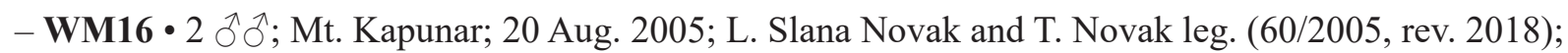
PMSL 1 §̄; Pernice; 20 Aug. 2005; L. Slana Novak and T. Novak leg. (75/2005, rev. 2018); PMSL • 1 क; ibid.; 27 Apr. 2008; L. Slana Novak and T. Novak leg. (167/2011, rev. 2017); PMSL • 1 q; Prevalov graben, Muta; 27 Apr. 2008; L. Slana Novak and T. Novak leg. (150/2011, rev. 2017); PMSL・ 1 q; Radlje ob Dravi; 2 Jul. 1939; (334/1983, rev. 2017); PMSL・ 1 q; ibid.; 15 May 1966; I. Leitinger leg. (510/1981, rev. 2018); PMSL・ 1 q; ibid.; (513/1981, JG det., TN rev. 2018); PMSL・ 1 đ; ibid.; 12 Jun. 1966; I. Leitinger leg. (514/1981, rev. 2018); PMSL・ 1 §; ibid.; (517/1981, rev. 2018); PMSL・ 1 क; ibid.; 10 Jul. 1966; I. Leitinger leg. (525/1981, JG det., TN rev. 2018); PMSL • 1 क; ibid.; 8 Aug. 1966;

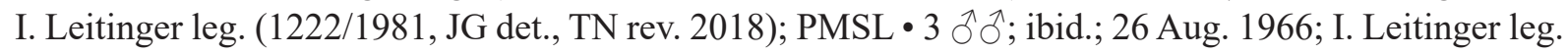
(511/1981, rev. 2018); PMSL・ 1 q; ibid.; (512/1981, JG det., TN rev. 2018); PMSL・ 2 q ; ibid.; (515/1981, JG det., TN rev. 2018); PMSL • 1 q; ibid.; (524/1981, JG det., TN rev. 2018); PMSL・ 1 \%; ibid.; 9 Sep. 1966; I. Leitinger leg. (516/1981, JG det., TN rev. 2018); PMSL・1 q; ibid.; (671/1981, rev. 2017); PMSL・ 1 क; ibid.; (152/1982, JG det., TN rev. 2018); PMSL • 1 q; ibid.; 8 Oct. 1973; T. Novak

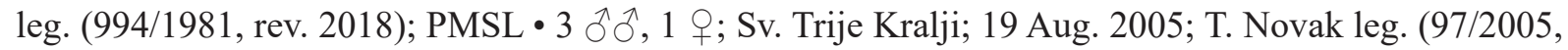
rev. 2017); PMSL • 1 O; ibid.; 20 Aug. 2005; L. Slana Novak and T. Novak leg. (82/2005, rev. 2017);

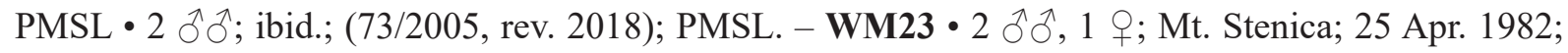
T. Novak; G. Kolar leg. (267/1982, JG det., TN rev. 2018); PMSL. - WM32 • 1 đ̊; Razbor, Ponikva; 9 Jun. 2015; T. Novak leg. (54/2015, rev. 2017); PMSL. - WM33 • 6 ô ðૈ, 7 o o ; Preloge; 17 Aug. 1983; L. Slana Novak and T. Novak leg. (LSN 73/1983, TN rev. 2018); PMSL. - WM34 • 1 9; Sv. Trije Kralji, the Pohorje Mts; 10 Aug. 1984; L. Slana Novak, M. Slana Novak and T. Novak leg. (LSN 250/1984, TN rev. 2018); PMSL. - WM35 • 1 क; Smolnik; 8 Aug. 2015; L. Slana Novak and T. Novak leg. (108/2015, TN rev. 2017); PMSL. - WM36・ 2 ふ઼, 2 q ; Ožbalt; 25 Oct. 2013; T. Novak leg. (45/2013, rev. 2017); PMSL. - WM43 • 1 क ; Brezje pri Poljčanah; 10 Apr. 1995; S. Brelih leg. (714/1998, rev. 2017); PMSL - 1 क; Zbelovo; 13 Jul. 2002; L. Slana Novak and T. Novak leg. (1158/2002, rev. 2018); PMSL. - WM45 - 1 9; Sv. Bolfenk, the Pohorje Mts; 27 Jul. 1987; F. Janžekovič leg. (5/1999, TN rev. 2018); PMSL •

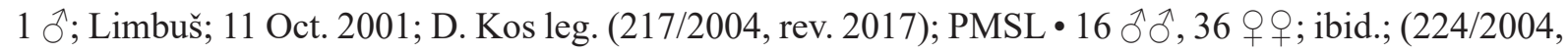

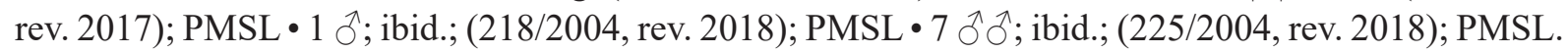
-WM46 • 1 q; Gaj nad Mariborom; 16 Apr. 1983; L. Slana Novak, M. Slana Novak and T. Novak leg. (LSN 64/1983, TN rev. 2018); PMSL • 1 q; ibid.; 20 Apr. 2008; L. Slana Novak and T. Novak leg. (164/2011, rev. 2018); PMSL • 1 juv.; Gaj nad Mariborom - Žavcarjev vrh; 1 Aug. 1981; T. Novak leg. (1084/1981, rev. 2019); PMSL • 1 क; Jurski vrh; 20 Apr. 2008; L. Slana Novak and T. Novak leg. (166/2011, rev. 2017); PMSL・ 2 $\widehat{\jmath}, 2$ 우; Kamniška graba; 20 Apr. 2008; L. Slana Novak and T. Novak leg. (129/2011, rev. 2018); PMSL・ 5 ふ઼ L. Slana Novak and M. Štangelj leg. (LSN 10/1983, TN rev. 2018); PMSL. - WM54 • 1 ô, 1 क; Marjeta na Dravskem polju; 8 Jun. 2015; P. Kozel leg. (57/2015, rev. 2017); PMSL. - WM55 • 1 क; Maribor Ruperče; 20 Apr. 1993; S. Brelih leg. (852/1998, rev 2018); PMSL. - WM64 • 1 đ̊; Krčevina pri Vurbergu; 15 Sep. 2015; T. Marinko leg. (134/2015, TN rev. 2017); PMSL・ 1 Ȯ; Placar; 16 Oct. 1994; M. Varžič leg. (17/1999, tev. 2018); PMSL. - WM65 - 1 Ö; Hum; 23 Apr. 2008; L. Slana Novak and T. Novak leg (130/2011, rev. 2017); PMSL・ 2 $\widehat{\jmath}, 2$ 우; Zgornja Voličina - Hrastovec; 2 Oct. 2002; T. Novak leg. (1284/2002, rev. 2018); PMSL. - WM74 • 1 ó; Hlaponci; 23 Apr. 1998; S. Brelih leg. (73/2001, rev.

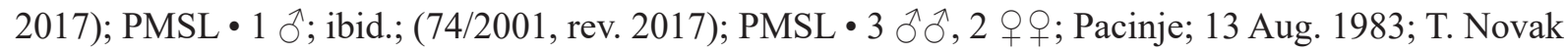
and L. Slana Novak leg. (LSN 60/1983, T. Novak rev. 2018); PMSL. - WM75 • 2 q ; Drbetinci; 28 Apr. 2002; L. Slana Novak and T. Novak leg. (731/2002, rev. 2017); PMSL・ 1 Ō, 1 क ; Kokolanjšček; 26 Sep. 2005; L. Slana Novak and T. Novak leg. (121/2005, rev. 2017); PMSL・ 1 ô, 1 + ; Velika Slavšina; 13 Oct. 2005; L. Slana Novak and T. Novak leg. (149/2005, rev. 2017); PMSL • 3 $\partial \hat{\jmath}$; Vitomarci; 2 Jun. 2002; L. Slana Novak and T. Novak leg. (756/2002, rev. 2018); PMSL. - WM76 • 1 ð; Kočki vrh; 30 Jun. 2002; T. Novak leg. (784/2002, rev. 2017); PMSL. - WM95 • 1 đ̂; Gresovščak; L. Slana Novak and T. Novak leg. (162/2012, TN and SL rev. 2017); PMSL. 


\section{Description}

Male (holotype)

BoDy. Length 1.94, width 1.43.

Chelicerae. Ch basal article (without Apo) length 0.58, 2.0 times as long as wide at $\mathrm{Ch}$ max width at dorsal hump. Ch-Apo elongated oval-trapezoid, shallowly bilobe apically, wider distally, with a prominent apical pinnacle posteriory. Ch-Apo height 0.21 , min width 0.08 (at the base) and max width 0.15 . Secretion field frontally. Distal article length 0.60, max width 0.25, movable finger length 0.28 (Figs 4H, 6H, 9H).

PediPALPS. Pa-Tr with dorsal margin conspicuously inclined distally. Pa-Fe conspicuously club-shaped (Pa-Fe min:max width 1:2.8) with dorsal margin bent in the middle and nearly straight anterior and posterior portions, and evenly arched ventral margin, with 3 stout spines in distal fourth. Pa-Ti hump low, inconspicuous (Figs 5H, 6H). Pa-Pt length:max width 1:4.1. Pa article lengths in Table 11.

PenIs. Pe length 1.47, base 0.48, glans 0.09, stylus 0.07 (Figs.7A, H, 8H).

LEGs. Pseudoarticle leg-Fe formula I-IV: 0-1-1-2. Tarsomere leg-Fe formula I-IV: 10-17-10-10. Leg article lengths in Table 11.

\section{Female}

BoDy. Length 2.11, width 1.65.

Chelicerae. Ch basal article width 0.63, 2.7 times as long as wide at dorsal hump, with nearly straight dorsal margin in front of indentation and ventral margin (Fig. 13G). Distal article length 0.64, max width 0.23 , movable finger length 0.27 .

PedipalPs. Pa-Tr with low dorsal margin, highest in the first third and straight posteriorly. Pa-Fe with conspicuous distal bonce, Pa-Pt, Pa-Ti and Pa-Ta elongated (Fig. 13G). Pa article lengths in Table 11.

Ovipositor. Ovipositor length 0.84 , Rec sem of one tubular and 12 slightly elongated vesicles (Fig. 11G).

LEGS. Leg article lengths in Table 11.

\section{Remarks}

Hadži (1927, sub N. bidentatum) made the first detailed drawings of $N$. $b$. schmidti ssp. nov. from Schmidt's collection. Prior to this study, all published evidence citing either $N$. bidentatum or $N$. b. bidentatum in Slovenia (all localities), Croatia (Zagreb) and NE Italy (all localities) refers to $N . b$. schmidti ssp. nov. Previously, all authors considered this subspecies to be within the range of variability of $N$. b. bidentatum (Roewer 1917, 1923; Hadži 1927, 1928, 1931; Gruber \& Martens 1968; Marcellino 1973; Martens 1978; Novak et al. 1984, 1995, 1996, 2002, 2017; Novak \& Gruber 2000; Komposch \& Gruber 2004; Novak 2004a, 2005b, 2005c). However, Roewer (1951) noticed that Hadži's individuals designated as $N$. bidentatum probably belonged to another species, while Novak \& Gruber (2000) considered $N$. b. schmidti ssp. nov. a possible hybrid $N$. b. bidentatum $\times N$. b. sparsum. The glans tip of a specimen from Leutschach/Lučane, $\left(46.67^{\circ} \mathrm{N}, 15.47^{\circ} \mathrm{E}, 350 \mathrm{~m}\right.$ a.s.1.; Gruber \& Martens 1968: fig. 24; Martens 1978: fig. 142), Austria, is the first detailed drawing of the glans in N. b. schmidti ssp. nov. See remarks under $N$. pluridentatum stat. nov., $N$. b. gruberi ssp. nov. $\times N$. b. schmidti ssp. nov. and $N$. b. martensi ssp. nov. $\times N$. b. schmidti ssp. nov.

\section{Distribution}

Austria, Italy, Croatia, Slovenia. Vertical distribution in Slovenia: 77-2108 m a.s.l. Type locality: Dolenja Sevnica, Poljane nad Škofjo Loko (46.12 ${ }^{\circ} \mathrm{N}, 14.15^{\circ} \mathrm{E}, 517 \mathrm{~m}$ a.s.1.), Slovenia. 
Table 11. Length of appendage-segments in millimeters in $N$. bidentatum schmidti Novak, Raspotnig \& Slana Novak ssp. nov. from Dolenja Sevnica, Slovenia: $\widehat{o}$ holotype (ㅇ).

\begin{tabular}{lccccccc}
\hline & Trochanter & Femur & Patella & Tibia & Metatarsus & Tarsus & Total length \\
\hline Pedipalp & $0.28(0.30)$ & $0.77(0.83)$ & $0.45(0.60)$ & $0.46(0.48)$ & - & $0.26(0.33)$ & $2.22(2.54)$ \\
Leg I & $0.21(0.25)$ & $0.86(0.85)$ & $0.33(0.35)$ & $0.63(0.60)$ & $0.93(1.01)$ & $0.95(0.99)$ & $3.91(4.05)$ \\
Leg II & $0.23(0.29)$ & $1.35(1.35)$ & $0.41(0.49)$ & $1.12(1.08)$ & $1.98(1.95)$ & $1.58(1.63)$ & $6.67(6.79)$ \\
Leg III & $0.22(0.18)$ & $0.86(0.85)$ & $0.35(0.35)$ & $0.59(0.60)$ & $1.08(1.04)$ & $0.87(0.78)$ & $3.97(3.80)$ \\
Leg IV & $0.22(0.26)$ & $1.32(1.33)$ & $0.41(0.40)$ & $0.82(0.81)$ & $1.60(1.81)$ & $1.11(1.08)$ & $5.48(5.69)$ \\
\hline
\end{tabular}

\section{Ecology}

Nemastoma b. schmidti ssp. nov. is a mesophilous colline to montane subspecies occupying most regions of Slovenia, except the southern and eastern territory, inhabiting various forest and scrub communities, from mixed hardwood riverine forests in the lowland to subalpine Nardion strictae Br.-B1. 1926 with isolated patches of Pinus mugo Turra and low-growing or krummholz communities. Phenology: adults eurychronous.

Nemastoma bidentatum sneznikensis Novak, Komposch, Slana Novak \& Raspotnig ssp. nov. urn:lsid:zoobank.org:act:7690FA07-5D60-4545-8660-282ADED3F576

Figs 2-3, 4I, 5I, 6I, 7I, 8I, 9I, 10I, 11I, 12I, 13H; Table 11

\section{Etymology}

The subspecies name 'sneznikensis' references Mt. Snežnik, southern Slovenia.

\section{Diagnosis}

Short-legged (leg II $<6 \mathrm{~mm}$ ) species of the $N$. $b$. complex, with slightly elongated-trapezoidal Ch-Apo, with row of low tubercles on Pa-Fe and with equilateral triangular glans. Rec sem of 18 balloon-like vesicles.

\section{Material examined}

\section{Holotype}

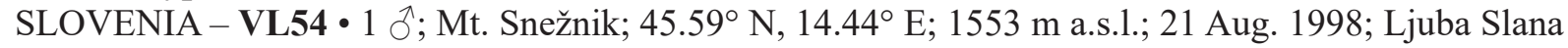
Novak and Tone Novak leg.; deep-humus in Dinaric Pinus mugo scrub in contact with the Dinaric Fagus sylvatica-Abies alba treeline litter sift; PMSL-Opiliones-TN 1494/1998.

\section{Paratypes}

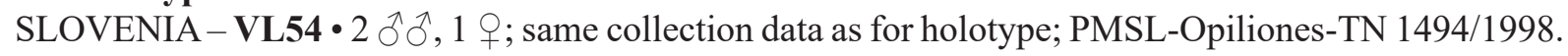

\section{Other material}

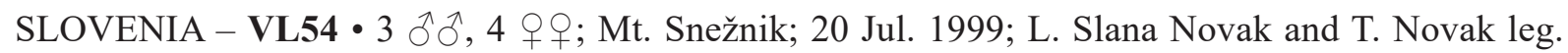
(185/1999, rev. 2009); PMSL.

\section{Description}

Male (holotype)

BoDY. Length 1.81, width 1.18.

Chelicerae. Ch basal article (without Apo) length 0.53, 2.7 times as long as wide at $\mathrm{Ch}$ max width at dorsal hump. Ch-Apo oval-trapezoid, wider distally, without apical pinnacle. Ch-Apo height 0.13, max width 0.13 , min width 0.08 (at the base). Secretion field frontally. Distal article length 0.51 , max width 0.17 , movable finger length 0.22 (Figs 4I, 6I, 9I). 
Table 12. Length of appendage-segments in millimeters in Nemastoma bidentatum sneznikensis ssp. nov. from Mt. Snežnik, Slovenia: $\widehat{\jmath}$ holotype (q).

\begin{tabular}{lccccccc}
\hline & Trochanter & Femur & Patella & Tibia & Metatarsus & Tarsus & Total length \\
\hline Pedipalp & $0.20(0.15)$ & $0.59(0.58)$ & $0.43(0.48)$ & $0.33(0.38)$ & - & $0.24(0.25)$ & $1.79(1.84)$ \\
Leg I & $0.19(0.18)$ & $0.73(0.62)$ & $0.31(0.28)$ & $0.52(0.51)$ & $0.79(0.96)$ & $0.74(0.63)$ & $3.28(3.18)$ \\
Leg II & $0.19(0.23)$ & $1.16(0.95)$ & $0.38(0.38)$ & $1.03(0.93)$ & $1.46(1.61)$ & $1.27(1.24)$ & $5.49(5.34)$ \\
Leg III & $0.18(0.17)$ & $0.70(0.78)$ & $0.29(0.27)$ & $0.53(0.52)$ & $0.86(1.04)$ & $0.63(0.57)$ & $3.19(3.35)$ \\
Leg IV & $0.17(0.18)$ & $1.06(0.98)$ & $0.33(0.28)$ & $0.74(0.73)$ & $1.42(1.44)$ & $1.01(0.92)$ & $4.73(4.53)$ \\
\hline
\end{tabular}

PEDIPALPS. Pa-Tr with dorsal margin slightly inclined distally. Pa-Fe slightly club-shaped (Pa-Fe min:max width 1:2.6) with terminal bonce, and 7 irregular granula in distal third. Pa-Pt length:max width $\sim 1: 4.3$. Pa-Ti slender (Figs 5I, 6I, 10I). Pa article lengths in Table 12.

Penis. Pe length 1.22, base 0.46, glans 0.09, stylus 0.04 (Figs 7I, 8I).

LEGS. Pseudoarticle leg-Fe formula I-IV: 0-1-1-2. Tarsomere leg formula I-IV: 9-14-6-8. Leg article lengths in Table 12.

\section{Female}

BoDy. Length 2.11, width 1.43.

Chelicerae. Ch basal article length 0.59, 2.7 times as long as wide at dorsal hump, with low-arched dorsal margin in front of indentation and straight ventral margin (Fig. 13H). Distal article length 0.66, max width 0.20 , movable finger length 0.30 .

PEDIPALPS. Pa-Tr low, with roof-like broken dorsal margin, highest in the first two fifths. Pa-Fe distally with low ventral hump, Pa-Pt, Pa-Ti and Pa-Ta elongated (Fig. 13H). Pa article lengths in Table 12.

OvIPOSITOR. Ovipositor length 0.62, Rec sem of 18 balloon-like vesicles (Fig. 11I).

LEGS. Leg article lengths in Table 12.

\section{Remarks}

Regarding external morphology, $N$. $b$. sneznikensis ssp. nov. is very similar to $N$. relictum, including the habitus, the similarly shaped $\mathrm{Pa}$ and relatively short legs. No individuals belonging to the subspecies were found in the last decade. See remarks on taxa of Nemastoma on Mt. Snežnik under N. b. gruberi ssp. nov.

\section{Distribution}

Despite over 30 collections, this taxon has been recorded only in a limited area on Mt. Snežnik. Endemic to Mt. Snežnik, southern Slovenia. Type locality: Mt. Snežnik (45.59 N, $14.44^{\circ}$ E, 1553 m a.s.1.), Slovenia.

\section{Ecology}

Nemastoma b. sneznikensis ssp. nov. is a psychrophilous subspecies. To date it has been recorded only in a cold, well-drained, deep mull humus Dinaric Pinus mugo scrub in contact with the Dinaric Fagus sylvatica-Abies alba treeline on calcareous soils. Likely, it inhabits other cold habitats, like scree and stone heap edges in contact with humus accumulations, as is known for N. relictum (Komposch 1999; Komposch \& Gruber 2004), and perhaps a humus-rich shallow subterranean habitat (SSH), i.e., Milieu Souterrain Superficiel (MSS) (Mammola et al. 2016; Halse 2018). Since this habitat is restricted to an area of less than $1 \mathrm{~km}^{2}$ on Mt. Snežnik, this subspecies is considered highly endangered. Phenology: probably eurychronous. 


\section{Hybrids among the subspecies of Nemastoma bidentatum}

\section{Remarks}

In Austria, a few localities are known where $N$. b. bidentatum lives in syntopy with $N$. relictum (Gruber \& Martens 1968; Martens 1978; in both papers sub N. b. relictum), and with N. b. sparsum (Komposch \& Gruber 2004; Komposch 2009). However, individuals of these three taxa do not interbreed and have been considered as potential species (Gruber \& Martens 1968; Martens 1978; Komposch \& Gruber 2004; Komposch 2009). In Slovenia, $N$. b. schmidti ssp. nov., with its central geographic position, interbreeds with all other adjacent subspecies: $N$. $b$. bidentatum in the North, and $N$. $b$. martensi ssp. nov., $N$. $b$. sneznikensis ssp. nov., $N$. $b$. gruberi ssp. nov. and $N$. $b$. sparsum from the Southwest to the Northeast of the country. Besides, $N$. $b$. gruberi ssp. nov. interbreed with $N$. b. martensi ssp. nov., and with $N$. $b$. sparsum. In Bosnia, $N$. pluridentatum stat. nov. has not been found; consequently, the genitalia have not been examined. Despite this, major differences from all the other known taxa within the $N$. $b$. complex allow it to be considered a separate species. This is because interbreeding of $N$. pluridentatum stat. nov., which might be a subalpine species, with the montane $N$. kozari sp. nov. from the same mountain is quite unlikely, given their great dissimilarity. However, data on taxa of Nemastoma in Bosnia and Herzegovina are nearly completely missing, allowing no further conclusions.

Although being morphologically variable, hybrids possess larger Ch-Apo than pure subspecies and are well distinguishable from these. In interbreeding subspecies, hybrids between the two subspecies frequently occur syntopically with individuals belonging to pure subspecies. Consequently, such a collection consists of either one or two pure subspecies and a series of hybrids with varying Ch-Apo shapes.

An overview of interbreeding relations among the taxa of the $N$. $b$. complex is presented in Table 13. In Slovenia, $N . b$. bidentatum and $N$. $b$. sparsum do not interbreed, since the areas are several tens of kilometres distant from each other. In contrast, we have found hybrids between all the adjacent subspecies (Fig. 3), except $N$. b. gruberi ssp. nov. $\times N$. b. schmidti ssp. nov. $\times N$. b. sparsum, which is expected in the meeting area of the three taxa. Besides, data on hybridization of $N$. b. sneznikensis ssp. nov. are too scarce to be properly evaluated in full. The zone of hybridization between $N$. $b$. schmidti ssp. nov. and $N$. b. sparsum is the widest, measuring up to $100 \mathrm{~km}$ in north-eastern Slovenia, while these zones are narrow to virtually non-identifiable among the others, and hybrids are accordingly scarce. Hybrids $N$. $b$. gruberi ssp. nov. $\times N$. $b$. schmidti ssp. nov. were found in a zone up to $10 \mathrm{~km}$ wide, while $N$. b. martensi ssp. nov. $\times N$. b. schmidti ssp. nov. and $N$. $b$. gruberi ssp. nov. $\times N$. $b$. martensi ssp. nov. are limited to zones a few kilometers wide. In Slovenia, there are no hybrids between allopatric $N$. b. martensi ssp. nov. and N. b. sparsum, while these are expected in Croatia.

Hybridization patterns were not simple clines in the transition zones between two subspecies. Instead, in some cases, morphologically various hybrids between the neighboring subspecies, sharing various ratios of the hybridizing subspecies characters (cf. Gruber \& Martens 1968; Martens 1978), appeared scattered across the whole width of the transition zone. In some places in the hybridization zone, individuals of both subspecies, together with hybrids of various intermittent stages, occurred syntopically.

Nemastoma bidentatum bidentatum Roewer, $1914 \times$ N. bidentatum schmidti ssp. nov. Figs 3, 9J, 10J.

\section{Diagnosis}

Typically, males with narrow quarter moon-like, apically bifid Ch-Apo, 2.4 times as high as wide, with wide, inconspicuous apical pinnacle posteriorly, and $\mathrm{Pa}-\mathrm{Fe} \sim$ intermediate between both subspecies.

\section{Material examined}

SLOVENIA - VM44 • 1 đ, 1 P; Gromov rob, Jelendol, Tržič; 23 Aug. 2017; T. Novak leg. (GR 5339, GR 5338); PMSL. - WM06 • 1 ô, 1 क; Mt. Košenjak; 27 Sep. 1981; T. Novak leg. (520/1981, rev. 
Table 13. Confirmed hybrids among the taxa of the $N$. bidentatum complex.

\begin{tabular}{|c|c|c|c|c|c|c|c|c|}
\hline & 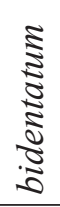 & 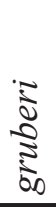 & 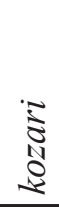 & $\begin{array}{l}\tilde{\Xi} \\
\vdots \\
\vdots \\
\vdots \\
\vdots\end{array}$ & 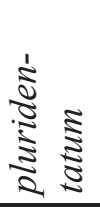 & 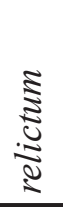 & 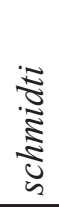 & 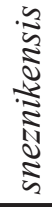 \\
\hline gruberi & & & & & & & & \\
\hline kozari & & & & & & & & \\
\hline martensi & & + & & & & & & \\
\hline $\begin{array}{l}\text { pluridentatum } \\
\text { relictum }\end{array}$ & & & & & & & & \\
\hline schmidti & + & + & & + & & & & \\
\hline sneznikensis & & & & & & & + & \\
\hline sparsum & & + & & & & & + & \\
\hline
\end{tabular}

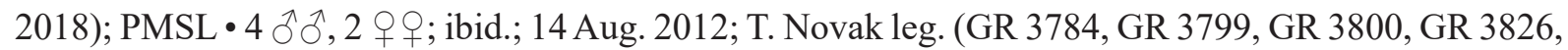
GR 3830, GR 3832); PMSL • 2 우; ibid.; 24 Aug. 2012; T. Novak leg. (153/2012, rev. 2018); PMSL

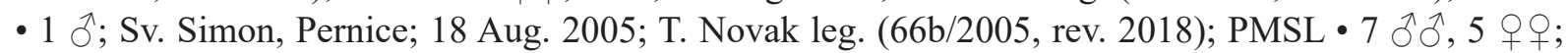
Sv. Urban, Kozji vrh nad Dravogradom; 24 Sep. 2005; L. Slana Novak and T. Novak leg. (127/2005,

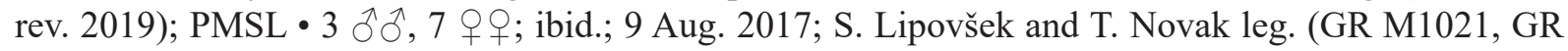
M1024, GR M1025, GR M1027, GR M1033, GR M1035-GR M1039); PMSL.

Nemastoma bidentatum bidentatum Roewer, $1914 \times$ N. bidentatum sparsum Gruber \& Martens, 1968

\section{Remarks}

Every allegation of hybrids $N$. b. bidentatum $\times N$. b. sparsum in Slovenia and Croatia (Gruber \& Martens 1968; Martens 1978; Novak \& Gruber 2000; Novak et al. 2002; Novak 2004b) refer either to N. b. schmidti ssp. nov. $\times N$. $b$. sparsum or $N$. b. gruberi ssp. nov. $\times N$. $b$. schmidti ssp. nov.; see these references for further remarks.

Nemastoma bidentatum gruberi ssp. nov. $\times N$. bidentatum martensi $\mathrm{ssp}$. nov.

Figs $3,9 \mathrm{~K}, 10 \mathrm{~K}$

\section{Diagnosis}

Typical male hybrids are characterized by $\mathrm{Ch}$ basal article with large hump in front of dorsal indentation, and broad, low quarter moon-like $\mathrm{Ch}$-Apo, $\sim 1.6$ times as high as wide, and slightly club-shaped, relatively thin $\mathrm{Pa}-\mathrm{Fe}$, and five, subequidistant, simple ventral Pa-Fe denticles in the frontal half of the Pa-Fe.

\section{Material examined}

CROATIA - WL00 • 8 ふึ ô, 11 q $q$; Debeli Lug, Jasenak; 8 Sep. 2009; (2/2012); PMSL. - WL20 •

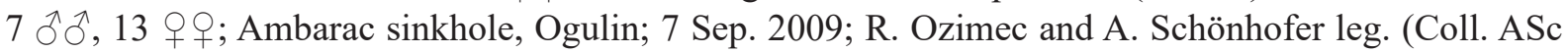
324, 1/2012); PMSL.

SLOVENIA - VL26 • 3 ภึ, 1 ค; Senožeče - Laže; 20 May 2012; L. Slana Novak and T. Novak leg. (50/2012, rev. 2019); PMSL. - VL33 • 2 §ึ, 3 우; Plazine, Starod; 25 Oct. 2012; L. Slana Novak and T. Novak leg. (194/2012, rev. 2018); PMSL. - VL54 • 1 ô; Travni Dolci, Mt. Snežnik; 12 Aug. 
2001; L. Slana Novak and T. Novak leg. (195b/2001, rev. 2020); PMSL • 1 đ̇; Mt. Snežnik; 12 Sep. 2018; L. Slana Novak and T. Novak leg. (116a/2018); PMSL・1 ð̇; ibid.; 25 Oct. 2019; T. Novak. leg. (138/2019); PMSL. - VL95 • 1 ô, 2 q o+; Mt. Kočevska Mala gora; 17 Aug. 1985; T. Novak, M. Slana Novak and L. Slana Novak leg. (LSN 98/1986, TN rev. 2019); PMSL. - VL96 • 1 đ;; Pečke - Mali vrh, Kočevski Rog; 20 Sep. 2001; B. Drovenik and A. Pirnat leg. (10/2005, rev. 2015); PMSL • 1 q; ibid.; (16/2005, rev. 2015); PMSL. - WL37 • 3 đ̂̉ ô, 2 우; Mirčev grič, Mt. Gorjanci; 29 Apr. 1995; S. Brelih leg. (780/1998, rev. 2019); PMSL.

\section{Nemastoma bidentatum gruberi ssp. nov. $\times N$. bidentatum martensi $\mathrm{ssp}$. nov. $\times N$. bidentatum schmidti ssp. nov.}

Figs 3, 9L, 10L

\section{Diagnosis}

Typical male hybrids are characterized by Ch-Apo with nearly symetrically convex ventro-anterior margin and straight posterior margin, and club-shaped, robust Pa-Fe with five simple, more or less equidistant ventral Pa-Fe spines in the frontal half, and Pa-Ti with small dorsal hump.

\section{Material examined}

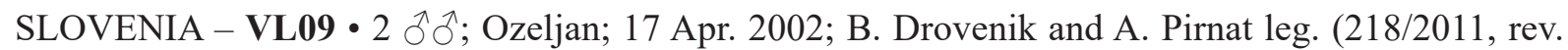
2017); PMSL. - VL26 • 3 ふぇ, 4 우; Laže, Razdrto; 1 Oct. 2011; L. Slana Novak and T. Novak leg. (358/2011, rev. 2017); PMSL.

Nemastoma bidentatum gruberi ssp. nov. $\times$ N. bidentatum schmidti ssp. nov.

Figs 3, 9M, 10M

Nemastoma bidentatum bidentatum - Gruber \& Martens 1968: 141 [partim: Dugo selo]. — Martens 1978: 107 [partim: Županja, Mt. Snežnik].

Nemastoma bidentatum bidentatum $\times$ Nemastoma bidentatum sparsum - Novak \& Gruber 2000: 285 [partim: Mt. Snežnik]. — Novak 2004b: 246 [partim: Dugo selo].

\section{Diagnosis}

In typical male hybrids, Ch-Apo is boomerang-shaped, $\mathrm{Pa}-\mathrm{Fe}$ club-shaped, but relatively thin, with four simple, equidistant ventral $\mathrm{Pa}-\mathrm{Fe}$ spines in the frontal half, $\mathrm{Pa}-\mathrm{Ti}$ without proximal dorso-medial hump.

\section{Material examined}

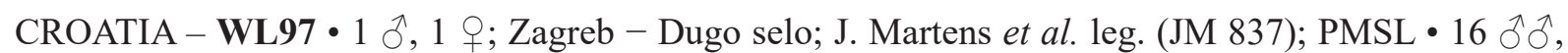
13 q ; ; ibid.; 9 Sep. 2009; A. Schönhofer leg. (ASc 342, 4/2012, ASc det., TN rev. 2018); PMSL. - XL06

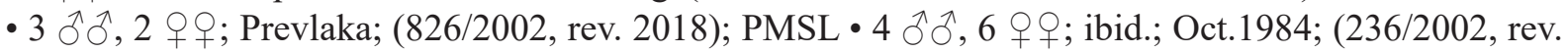

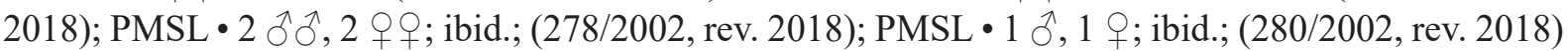
• 1 ô, 1 ㅇ; ibid.; 15 Aug. 1985; (LSN 28/1996, TN rev. 2018); PMSL・ 1 ㅇ; ibid.; (891/2002, rev. 2018);

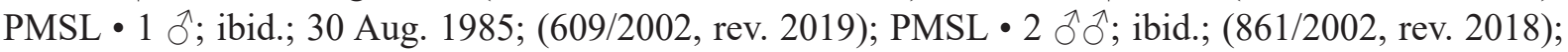

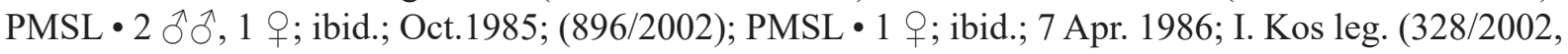
rev. 2018); PMSL・ 1 ơ; ibid.; (843/2002, rev. 2018); PMSL.

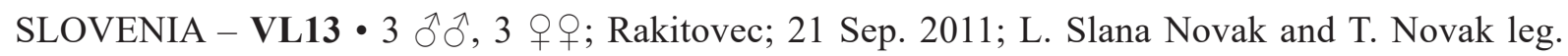

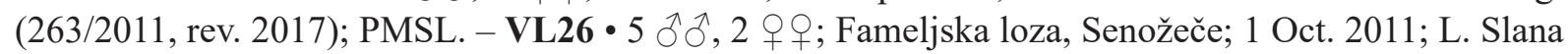
Novak and T. Novak leg. (350/2011, rev. 2017); PMSL・ 1 ô; Senožeče - Vremščica; 1 Oct. 2011; L. Slana Novak and T. Novak leg. (355/2011, rev. 2017); PMSL. - VL27 • 3 ô $\widehat{~}, 1$ \&; Mt. Nanos; 1 Sep. 


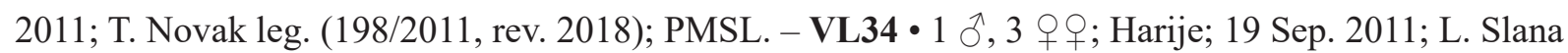
Novak and T. Novak leg. (224/2011, rev. 2015); PMSL • 1 क; Petelinje; 27 Apr. 1994; S. Brelih leg. (815/1998, rev. 2015); PMSL. - VL58 • 2 đ̂̉ (83/2001, rev. 2015); PMSL. - VL67 • 1 ô, 2 우우 Lužarji; 25 Aug. 2011; T. Novak leg. (168/2011, rev. 2018); PMSL. - VL78 • 1 क; Mt. Limberk; Jun.1998; I. Bertoncelj leg. (1544/1998, rev. 2009); PMSL

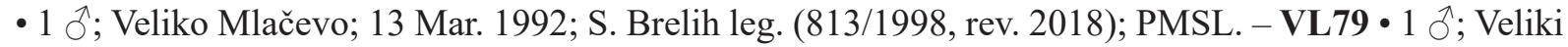
Lipoglav; 8 Jul. 1986; L. Slana Novak and T. Novak leg. (472/2002, rev. 2018); PMSL. - VL84 • 1 ○,, 2 O+ ; Pleš, Borovec pri Kočevski Reki; 19 Sep. 2013; P. Kozel and T. Novak leg. (27/2013, rev. 2015); PMSL. - VL88 • 1 ðે, 1 क; Krka, Krška vas pri Muljavi; 7 Jul. 1986; L. Slana Novak and T. Novak leg. (474/2002, rev. 2018); PMSL • 1 +; ibid.; Jun. 1998; V. Kovačič leg. (1444/1998, rev. 2017); PMSL.

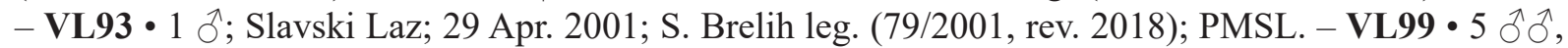
1 P; Cirnik; 27 May 1984; L. Slana Novak, M. Slana Novak and T. Novak leg. (LSN 249/1984, TN rev. 2015); PMSL • 1 क; Temenica; 4 Mar. 1994; S. Brelih leg. (680a/1998, rev. 2018); PMSL. - WL04 - 5 ô; Brezovica pri Predgradu; 17 Aug. 1985; L. Slana Novak, M. Slana Novak and T. Novak leg. (147/1997, rev. 2018); PMSL. - WL05 • 5 đ̊ đૈ; Črmošnjice; 18 Aug. 1985; L. Slana Novak, M. Slana Novak and T. Novak leg. (152/1997, rev. 2018); PMSL. - WL06 • 1 O , 1 ; ; Baza 20, Kočevske Poljane; 17 Aug. 1985; L. Slana Novak, M. Slana Novak and T. Novak leg. (LSN 76/1986, TN rev. 2018); PMSL• 2 ổ; Podstene, Kočevski Rog; 19 Jun. 1986 (672/2002, rev. 2017); PMSL. - WL08 • 1 ㅇ; Velika jama nad Trebnjem cave, Cad. No. 104, Mokronog; 28 Jul. 2009; M. Zagmajster leg. (PK and TN 6/2020); PMSL. - WL09 • 1 ठ̊; Šentrupert; 28 Feb. 1994; S. Brelih leg. (825/1998, rev. 2018); PMSL. - WL19

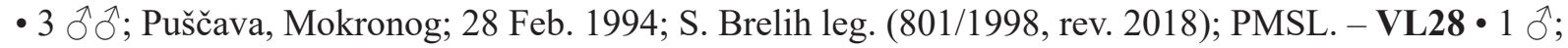
Mt. Homski hrib, Sela pri Zburah; Jun. 1997; D. Vrček leg. (1709/1998); PMSL. - WL29 • 1 ภ, 2 q 0 ; Dolenji Boštanj; 15 Aug. 2007; L. Slana Novak and T. Novak leg. (73/2007, rev. 2015); PMSL. - WM01 - 1 Ō, 6 우; Mt. Javor, Znojile; 21 Jul. 2002; L. Slana Novak and T. Novak leg. (1194/2002, rev. 2018); PMSL. - WM40 • 1 §̃; Lesično; 13 Jul. 2002; L. Slana Novak and T. Novak leg. (1130/2002, rev. 2018);

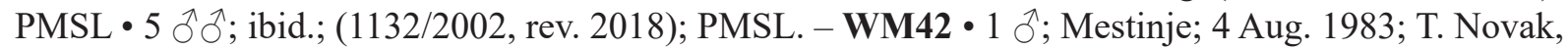
M. Štangelj and L. Slana Novak leg. (LSN 44/1983, TN rev. 2015); PMSL.

Nemastoma bidentatum gruberi ssp. nov. $\times$ N. bidentatum sparsum Gruber \& Martens, 1968

Figs $3,9 \mathrm{~N}, 10 \mathrm{~N}$

\section{Diagnosis}

Typical male hybrids with elongated Ch-Apo with knee-like flexion of frontal margin, and equidistant triangular upper portion, and club-shaped, but relatively thin $\mathrm{Pa}-\mathrm{Fe}$, with tubercle tightly in front of the first spine of four simple, equidistant ventral spines in distal Pa-Fe half, either pointed or almost without the tip.

\section{Material examined}

CROATIA - XL03 • 1 ふ̊; Jezero Posavsko, Suša; 3 Dec. 1985; (61/2019); PMSL.

Nemastoma bidentatum martensi ssp. nov. $\times N$. bidentatum schmidti ssp. nov.

Figs $3,9 \mathrm{O}$

Nemastoma (Stridulostoma) seliskari Hadži, 1973a: 48, figs 33c, 37a-e.

Nemastoma bidentatum bidentatum - Martens 1978: 107 [partim: Nemastoma (Stridulostoma) seliskari Hadži, 1973].

Nemastoma bidentatum bidentatum $\times$ N. bidentatum sparsum - Novak \& Gruber 2000: 286 [partim: Novo mesto, Mt. Mirna gora]. 
Nemastoma bidentatum pluridentatum - Schönhofer 2013: 36 [partim: Nemastoma (Stridulostoma) seliskari Hadži, 1973, and Nemastoma seliskari Hadži, 1973].

\section{Diagnosis}

Typical male hybrids with Ch basal article with large hump in front of dorsal indentation, Ch-Apo with knee-like frontal flexion and narrowly drawn out upper portion, Pa-Fe club-shaped, but thinner than in N. b. schmidti ssp. nov., with four simple, equidistant ventral spines in distal Pa-Fe half, and Pa-Ti with minute hump.

\section{Material examined}

SLOVENIA - VL15•1 đ̊; Prelože pri Lokvi; 27 Sep. 2011; L. Slana Novak and T. Novak leg. (310/2011); PMSL. - VL35 • 3 $\widehat{\jmath}$, 5 우; Ribnica - Ostrožno Brdo; 27 Sep. 2011; L. Slana Novak and T. Novak leg. (324/2011, rev. 2015); PMSL. - VL53 • 1 đ̧; Zabiče; 27 Sep. 2011; L. Slana Novak and T. Novak leg. (301/2011, rev. 2015); PMSL. - WL05 • 1 ठ̊; Mt. Mirna gora; 28 Jul. 1948; A. Seliškar leg; PMSL. -WL17 • 1 ठ̊; Novo Mesto; Karaman leg. (107/1983 microscopic preparation, JH det., TN rev. 2017); PMSL.

\section{Remarks}

In Hadži's original preparation, labelled "Nemastoma bidentatum Roewer pulli", with two cover glasses on the same microscope slide, there are two males and a female. One male corresponds to the type specimen of Nemastoma (Stridulostoma) seliskari Hadži, 1973 (Hadži 1973a). For this species, Hadži (ibid.) founded a new subgenus Stridulostoma based on the existence of an alleged "stridulatory organ" on the Fe III (Hadži 1973a: figs 37č-d). Gruber (1976: 797) explained that the stridulatory organ alone does not justify establishing of a new subgenus, especially because this new species differs from N. (Lugubrostoma) triste pluridentatum Hadži, 1973 exclusively by possession of this organ, and accordingly, synonymized Stridulostoma with Nemastoma. Following these remarks, Schönhofer (2013: 36) synonymized $N$. seliskari with $N$. b. pluridentatum. We found that the portion of the Fe III, identified by Hadži (1973a) as a stridulatory organ in the type specimen, is in fact a depression-damage, caused by desiccation. Moreover, such damage is present in nearly all (sic!) the other femora of this freshly moulted specimen, but nothing resembles the fine lamellated structure shown in Hadži's drawing (1973a: figs $37 \check{c}-d$ ). No similar structure was found in the specimens of Nemastoma collected later on Mt. Mirna gora. One might imagine that a careless inspection under a microscope at low magnification could result in the illusion of a 'stridulatory organ'. However, the drawing of its lamellae cannot be considered anything but a pure fabrication. Moreover, Pa-Fe in Hadži (1973a: fig. 33c), being under the same cover glass in the preparation, belongs to the same specimen, although Hadži (ibid.) ascribed it to N. (Lugubrostoma) triste pluridentatum. See remark under $N$. pluridentatum stat. nov.

Nemastoma bidentatum schmidti ssp. nov. $\times$ N. bidentatum sneznikensis ssp. nov.

\section{Diagnosis}

In typical male hybrids, Ch basal article oblong, sneznikensis-like, with Ch-Apo with small apical pinnacle posteriorly, Pa-Fe club-shaped, thinner than in $N$. $b$. schmidti ssp. nov., with three simple ventral spines in distal Pa-Fe half, Pa-Pt schmidti-like, Pa-Ti with inconspicuous tubercle.

\section{Material examined}

SLOVENIA - VL54 • 1 đ̊; Mt. Snežnik; 14 Sep. 2019; L. Slana Novak and T. Novak leg. (GR 5468); PMSL. 


\section{Remarks}

To date, only one male has been found.

Nemastoma bidentatum schmidti ssp. nov. $\times$ N. bidentatum sparsum Gruber \& Martens, 1968 Figs 3, 9P, $10 \mathrm{O}$

Nemastoma triste - Roewer 1917: 149: [partim: Celje/Cilli, Maribor/Marburg].

Nemastoma bidentatum bidentatum $\times N$. bidentatum sparsum - Gruber \& Martens 1968: 142, 147, figs 12-13 [partim: Novo mesto; Županja]. — Martens 1978: 107, fig 146 [partim: Županja; Novo mesto, Mt. Snežnik]. - Novak \& Gruber 2000: 286 [partim: Novo mesto]. — Novak et al. 2002: 136 [partim: Ptuj]. — Novak 2004b: 246 [partim: Županja].

Nemastoma bidentatum ssp. - Novak \& Gruber 2000: 286 [partim: Celje, Maribor].

\section{Diagnosis}

Hybrids exhibiting varying Ch-Apo morphology, but with knee-like flexion of anterior margin, and either being relatively tiny with more or less elongated superior portion or more massive with superior portion drawn out in tiny apical portion, and club-shaped $\mathrm{Pa}-\mathrm{Fe}$ with intermediate form with respect to both subspecies.

\section{Material examined}

SLOVENIA - VM91 • 2 ồ 4 4 क ; Čemšemik; 21 Jul. 2002; L. Slana Novak and T. Novak leg. (1183/2002, rev. 2018); PMSL・ 1 ô, 1 q; Jesenovo - Znojile; 21 Jul. 2002; L. Slana Novak and T. Novak leg. (1187/2002, TN rev. 2018); PMSL・ 6 đ̊̄, 5 o $O$; Mt. Čemšeniška planina; 21 Jul. 2002; L. Slana Novak and T. Novak leg. (1191/2002, rev. 2018); PMSL • 2 q ; Zaplanina, Ločica pri Vranskem; 21 Jul. 2002; L. Slana Novak and T. Novak leg. (1210/2002, rev. 2018); PMSL・ 1 Ô, 5 우; ibid.; (1179/2002, rev. 2018); PMSL. - VM92 • 4 ô ô, 9 q 9 ; Ločica pri Vranskem; 10 Aug. 1985; L. Slana Novak, M. Slana Novak and T. Novak leg. (LSN 101/1986, TN rev. 2018); PMSL. - WM01・ 7 §o , 5 q $\odot$; Marija Reka; 21 Jul. 2002; L. Slana Novak and T. Novak leg. (1186/2002, rev. 2018); PMSL. - WM05・4 ô ô, 7 우 Slovenj Gradec; 9 Oct. 1983; T. Novak and L. Slana Novak leg. (LSN 113/1983, TN rev. 2018); 3 $\widehat{\partial}$, 4 우; ibid.; 6 Jul. 2003; L. Slana Novak and T. Novak leg. (190/2003, rev. 2018); PMSL. - WM06 • 1 Ĵ, 2 9 o ; Dravograd; 25 Oct. 1995; S. Hudrap and M. Pavlin leg. (11/1996, rev. 2018); PMSL. - WM10

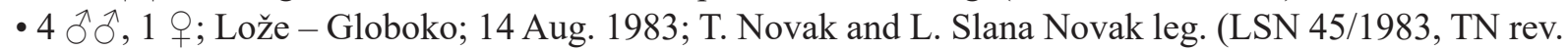
2018); PMSL. - WM11 • 1 o, 2 o o ; Brdce; 22 Apr. 1995; S. Brelih leg. (758/1998, rev. 2018); PMSL - 1 Ĵ, 3 우; Udmat; 15 Aug. 1983; T. Novak and L. Slana Novak leg. (LSN 47/1983, TN rev. 2018);

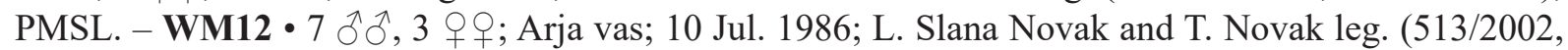
rev. 2018); PMSL • 1 ô, 1 q; Velika Pirešica; 10 Jul. 1986; L. Slana Novak and T. Novak leg. (452/2002,

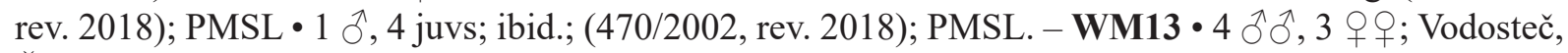
Črnova; 10 Jul. 1986; L. Slana Novak and T. Novak leg. (453/2002, rev. 2018); PMSL • 2 ô ; ibid.; 22 Apr. 1995; S. Brelih leg. (772/1998, rev. 2018); PMSL・ 1 ô, 1 q; ibid.; 21 Apr. 2002; L. Slana Novak and T. Novak leg. (737/2002, rev. 2019); PMSL. - WM16 • 2 $\widehat{\partial}$ ô, 3 우; Sv. Trije Kralji, Muta; 20 Aug.

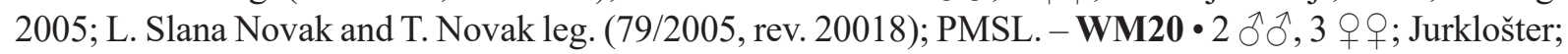
15 Aug. 1983; L. Slana Novak and T. Novak leg. (LSN 53/1983, TN rev. 2018); PMSL. - WM21 • 1 ô,

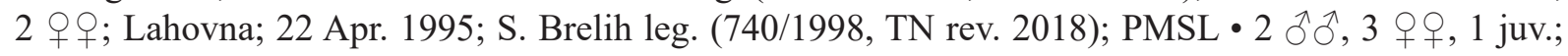
Trobni dol; 16 Aug. 1983; T. Novak and L. Slana Novak leg. (LSN 48/1983, TN rev. 2018); PMSL. -

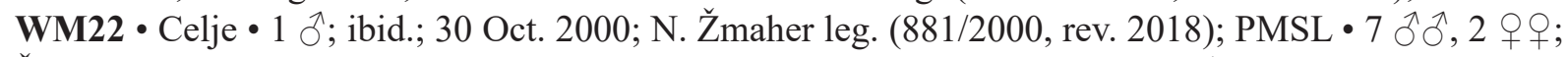
Šmartinsko jezero; 25 Mar. 1995; S. Brelih leg. (704/1998, rev. 2018); PMSL・ 1 Ô, 4 o+ $O$; Vojnik; 17 Aug. 1983; T. Novak and L. Slana Novak leg. (LSN 58/1983, TN rev. 2018); PMSL. - WM23 • 1 ð, 1 市;

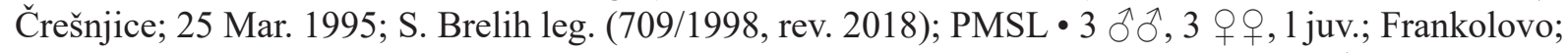
17 Aug. 1983; L. Slana Novak and T. Novak leg. (LSN 76/1983, TN rev. 2018); PMSL・ 1 ô;; Mt. Konjiška 
gora; 14 Jun. 1981; V. Kuštor, T. Novak leg. (1120/1981, rev. 2018); PMSL・ 1 ふૈ, 2 q ; Mt. Stenica; 30 May 1981; T. Novak, V. Kuštor leg. (228/1981, rev. 2018); PMSL・ 1 q; Stenica; 24 Jul. 2002; B. Drovenik and A. Gergeli leg. (1138/2018); PMSL • 1 क; ibid.; 24 Jul.-21 Aug. 2002; B. Drovenik and A. Gergeli leg. (PK 73/2012, TN rev. 2018); PMSL • 1 क; ibid.; (PK 12/2013, TN rev. 2018); PMSL• 1 ô; ibid.; 21 Aug. 2002; B. Drovenik and A. Gergeli leg. (1071/2018); PMSL • 1 \% ; ibid.; 21 Aug. -16 Sep.

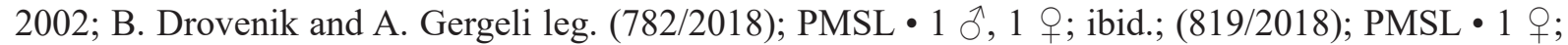
ibid.; (856/2018); PMSL・ 1 ภ, 1 क; ibid.; (804/2018); PMSL • 1 q; ibid.; 16 Sep.-14 Oct. 2002; B. Drovenik and A. Gergeli leg. (817/2018); PMSL 1 क; ibid.; 14 Oct. 2002; B. Drovenik and A. Gergeli leg. (361/ 2018); PMSL • 1 q; ibid.; (783/2018); PMSL・ 1 ô, 1 क; ibid.; (794/2018); PMSL・ 1 ô, 1 क; ibid.; (814/2018); PMSL・ 1 क; ibid.; (824/2018); PMSL・ 1 क; ibid.; (831/2018); PMSL・ 1 क; ibid.; (846/2018); PMSL・ 1 §̊; ibid.; 11 Nov. 2002; B. Drovenik and A. Gergeli leg. (208/2018); PMSL・ 1 juv.; ibid.; (266/2018); PMSL 1 ô; ibid.; 11 Nov. 2002-4 Mar. 2003; B. Drovenik and A. Gergeli leg. (694/2018); PMSL・ 1 o; ibid.; (698/2018); PMSL・ 2 q of; ibid.; (701/2018); PMSL・ 1 ô, 1 q; ibid.; (710/2018); PMSL・ 1 O ; ibid.; (716/2018); PMSL・ 1 O ; ibid.; (721/2018); PMSL・ 1 ô; ibid.; (724/2018); PMSL・ 3 ô $\widehat{\partial}, 2$ 우; ibid.; (729/2018); PMSL・ 1 ô; ibid.; 8 Apr. 2003; B. Drovenik and A. Gergeli leg. (267/ 2018); PMSL • 3 juvs; ibid.; (271/2018); PMSL • 1 ô;; ibid.; 8 Apr. 2003; B. Drovenik and A. Gergeli leg. (189/2018); PMSL • 1 juv.; ibid.; (266/2018); PMSL. - WM24 • 1 §, 1 क ; Jurgovo; 24 Jul.-3 Aug. 2005; A. Kapla leg. (178/2005, rev. 2018); PMSL • 3 ô ô; Resnik; 12 Aug. 1984; L. Slana Novak, M. Slana Novak and T. Novak leg. (LSN 252/1984, TN rev. 2018); PMSL. - WM25 • 3 ô đ̂; Glažuta, Mislinjski jarek; 9 Sep. 2017; M. Delakorda, S. Lipovšek, L. Slana Novak and T. Novak leg. (107/2018); PMSL • 1 क; ibid.; 19 Sep. 2018; S. Lipovšek, P. Kozel and T. Novak leg. (132/2018); PMSL・ 1 đ̊; Ribnica na Pohorju; 4 Jul. 1939; J. Hadži leg. (145/1984, rev. 2018); PMSL. - WM30 • 1 đ, 1 o;; Loke - Lažiše; 11 Jul. 2003; T. Novak leg. (154/2003, rev. 2018); PMSL. - WM31・3 ðð, 2 $+q$; Bezovje pri Šentjurju; 16 Aug. 1983; L. Slana Novak and T. Novak leg. (LSN 56/1983, TN rev. 2018); PMSL・1 ð̊; Slivniško jezero; 7 Apr. 1995; S. Brelih leg. (701/1998, rev. 2018); PMSL. - WM33 • 3 ðð, 2 우우, 1 juv.; Tepanje; 17 Aug. 1983; T. Novak and L. Slana Novak leg. (LSN 62/1983, TN rev. 2018); PMSL・ 2 ठ઼’; Žiče monastery; 25 Mar. 1995; S. Brelih leg. (706/1998, rev. 2018); PMSL. - WM40・1 ठ̊; Lesično; 13 Jul. 2002; L. Slana Novak and T. Novak leg. (1130/2002, rev. 2018); PMSL. - WM41 • 1 O̊, 5 + ; Podčetrtek; 13 Aug. 1983; L. Slana Novak and T. Novak leg. (LSN 51/1983, TN rev. 2018); PMSL・ 2 ふึ ibid.; 7 Jul. 2012; T. Novak leg. (51/2012, rev. 2018); PMSL・ 2 ổ 0 , 3 우; Virštajn; 7 Apr. 1995; S. Brelih leg. (699/1998, rev. 2018); PMSL. - WM42 - 1 क; Mt. Boč; 17 Apr. 1982; T. Novak and G. Kolar leg. (125/1982, rev. 2018); PMSL・ 2 $\widehat{\jmath}, 1$ q; ibid.; 16 Oct. 1983; L. Slana Novak and T. Novak leg. (LSN

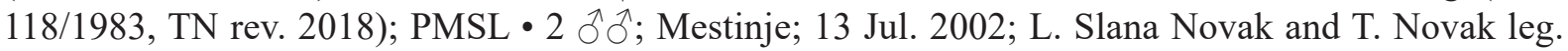

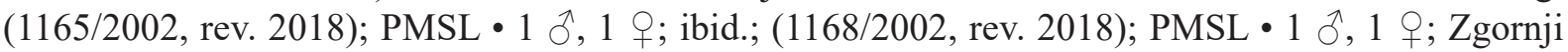
Gabrnik; 11 Oct. 1997; L. Slana Novak and T. Novak leg. (412/1998, rev. 2018); PMSL. - WM43 •

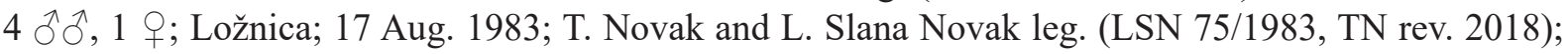

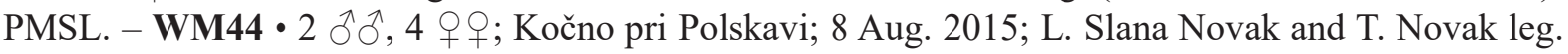
(110/2018); PMSL. - WM45 • 1 ð’; Limbuš; 11 Oct. 2001; D. Kos leg. (226/2004, rev. 2018); PMSL. -WM52 - 1 O; Sv. Donat, Donačka gora; 16 Sep. 2002; B. Drovenik and A. Pirnat leg. (247/2018); PMSL • 1 क; ibid.; (248/2018); PMSL • 1 O; ibid.; 14 Oct. 2002; B. Drovenik and A. Pirnat leg. (1032/2018); PMSL・ 6 đิ đ, 2 우, 2 juvs; Tuncovec; 4 Aug. 1983; T. Novak, M. Štangelj and L. Slana

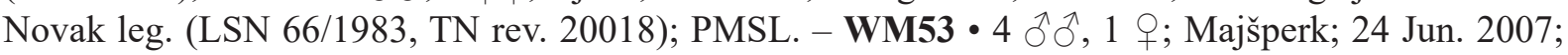
T. Novak leg. (43/2018); PMSL・ 4 đ̄ ô, 1 q; Pečke; 10 Apr. 1995; S. Brelih leg. (713/1998, rev. 2018);

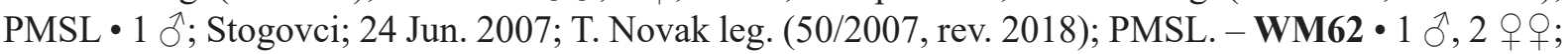
Dobovec pri Rogatcu; 7 Oct. 2001; T. Novak leg. (605/2002, rev. 2007); PMSL • 2 ổ; ibid.; 16 Aug. 2003; J. Ravljen and T. Novak leg. (807/2003, rev. 2018); PMSL 4 o $\widehat{\jmath}$; ibid.; 16 Aug. 2003; J. Ravljen, L. Slana Novak and T. Novak leg. (812/2003, rev. 2007); PMSL・ 1 ô, 2 q o ; ibid.; 30 Sep. 2012; T. Novak leg. (63/2015); PMSL・ 7 ふ઼, 4 우, 1 juv.; Kozminci; 6 Aug. 1983; T. Novak, M. Štangelj and L. Slana Novak leg. (LSN 61/1983, TN rev. 2018); PMSL • 1 क; Mt. Macelj; Jun. 1980; V. Kuštor and T. Novak leg. (LSN 5/1983, TN rev. 2018); PMSL • 2 q ; Žale, Dobrina; 24 Apr. 1998; S. Brelih leg. (85/2001, 


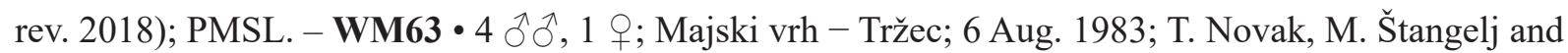

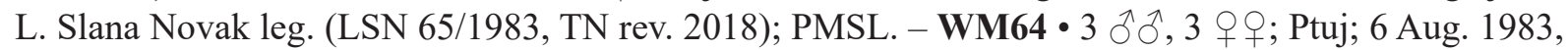
T. Novak, M. Štangelj and L. Slana Novak leg. (LSN 77/1983, TN rev. 2018); PMSL. - WM65 • 2 ô ō; Selca; 14 Jun. 1984; L. Slana Novak, M. Slana Novak and T. Novak leg. (LSN 238/1984, TN rev. 2018); PMSL・ 1 đ̂, 2 우; Zgornja Voličina; 23 Apr. 1998; S. Brelih leg. (124/2001, rev. 2018); PMSL. - WM73 • 1 ô, 4 우; Berinjak; 24 Jun. 2007; T. Novak leg. (36/2007, rev. 2018); PMSL・ 1 ô, 8 o o ; Borl castle, Dolane; 7 Aug. 1983; L. Slana Novak, M. Štangelj and T. Novak leg. (LSN 59/1983, TN rev. 2018); PMSL・ 1 ô, 2 + ; Ljubslava, Podlehnik; 24 Jun. 2007; T. Novak leg. (49/2007, TN rev. 2018); PMSL

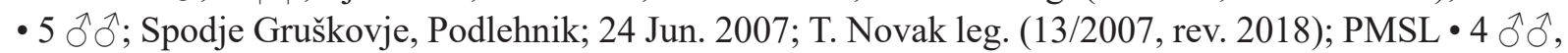
1 P; Vareja; 6 Aug. 1983; T. Novak, M. Štangelj and L. Slana Novak leg. (LSN 78/1983, TN rev. 2018);

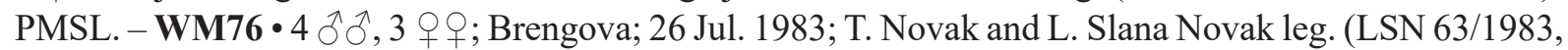
TN rev. 2018); PMSL・ 2 ô đે, 1 क ; Kočki vrh; 30 Jun. 2002; T. Novak leg. (783/2002, rev. 2018); PMSL

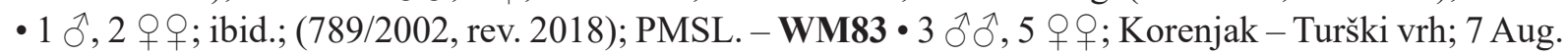
1983; T. Novak, M. Štangelj and L. Slana Novak leg. (LSN 55/1983, TN rev. 2018); PMSL. - WM84 • 4 ડ̄ô, 3 우; Vudina, Velika Nedelja; 29 May 2001; P. Igerc and T. Novak leg. (22/2001, rev. 2018); PMSL. - WM85 - 2 우; Radoslavci; 10 Sep. 1983; T. Novak, M. Slana Novak and L. Slana Novak leg. (LSN 117/1983, TN rev. 2018); PMSL. - WM94 • 2 $\widehat{\jmath}, 3$ 우; Pavlovci; 2 Apr. 1983; T. Novak and L. Slana Novak leg. (LSN 6/1983, TN rev. 2018); PMSL. - WM95 - Noršinci pri Ljutomeru; 27 Aug. 1983; T. Novak and L. Slana Novak leg. 2 $\widehat{\jmath} \widehat{\jmath}, 2$ 우우 (LSN 109/1983, TN rev. 2018); PMSL. - WM95

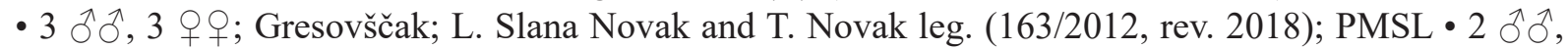
2 우; Slamnjak, Ljutomer; 10 Jun. 1984; L. Slana Novak and T. Novak leg. (LSN 241/1984, rev. 2018); PMSL.

\section{Remarks}

To date, $N$. b. schmidti ssp. nov. has been reported under $N$. $b$. bidentatum, and $N$. $b$. gruberi ssp. nov. under $N$. b. sparsum. Since hybrids $N$. b. bidentatum $\times N$. b. sparsum have not been identified in any locality, all these published data likely refer either to $N$. $b$. schmidti ssp. nov. $\times N$. $b$. sparsum or $N$. b. gruberi ssp. nov. $\times N$. b. schmidti ssp. nov. Nemastoma $b$. bidentatum referred from Germany (Schönhofer \& Holle 2007) is a hybrid, probably $N$. b. schmidti ssp. nov. $\times N$. b. sparsum. Since both $N$. b. sparsum and $N$. b. gruberi ssp. nov. inhabit the lowland in Croatia, hybrids south of the Sava River or nearby probably belong to $N$. $b$. gruberi ssp. nov. $\times N$. $b$. schmidti ssp. nov.

\section{Identification key to taxa within the Nemastoma bidentatum Roewer, 1914 complex}

The identification key refers to species of the $N$. bidentatum complex, and subspecies of $N$. bidentatum s. str. including typical male hybrids. Female hybrids show intermediate traits and are not considered in the key. Figs 4-5 summarize the written information visually throughout the key, and are therefore not referred in the key. Detailed morphology is presented in Figs 6-13. Consult characters presented in Figs 6-10 and 13 (pure taxa), and Figs 11-12 (pure taxa and hybrids, males) in support.

1. $\delta^{\lambda}$

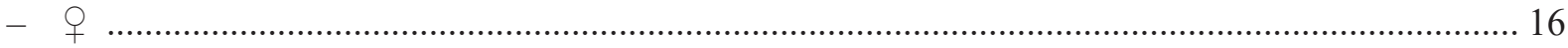

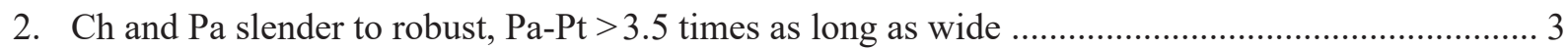

- Ch and Pa very robust, $\mathrm{Pa}-\mathrm{Pt}<2.8$ times as long as wide (Fig. 12D)

N. pluridentatum (Hadži, 1973) stat. nov.

3. Pa-Fe unarmed or with series of small tubercles, glans isosceles triangular

- Pa-Fe with either spines, thorns, pointed tubercles or 4 long, rigid, subequidistant setae, glans variously shaped 
4. Ch-Apo comb-like, Pa-Fe unarmed, without terminal bonce, Pa-Pt $\sim 4.0$ times as long as wide (Fig. 12C) N. relictum Gruber \& Martens, 1968 stat. nov.

- Ch-Apo rounded-trapezoidal, Pa-Fe with series of $\sim 7$ minute irregular tubercles, with terminal bonce, $\mathrm{Pa}-\mathrm{Pt}>4.8$ times as long as wide (Fig. 12I) N. b. sneznikensis ssp. nov.

5. Ch-Apo variously shaped, Pa-Fe with spines, thorns, pointed denticles or 4 long, rigid setae on low protrusions

- Ch-Apo low-trapezoid, Pa-Fe slightly club-shaped, with 6-11 medio-ventral pointed denticles, glans isosceles triangular (Fig. 12G)

N. b. martensi ssp. nov.

6. Pa-Fe robust, very club-shaped (Pa-Fe max:min width $>2.5: 1$ ) and ventrally bent (e.g., Figs 10J, O, 12A, F, H)

- Pa-Fe moderately club-shaped $(\mathrm{Pa}-\mathrm{Fe}$ max:min width $<2.4: 1)$ and ventrally bent ....................... 13

7. Pa-Ti with small medio-dorsal hump, glans truncated terminally ................................................. 8

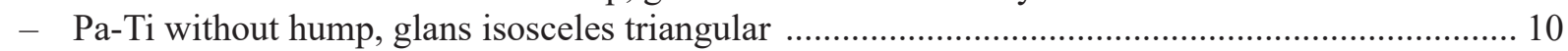

8. Ch-Apo either broadly quarter moon-like or elongated oval-trapezoid ......................................... 9

- Ch-Apo narrowly quarter moon-like, $\sim 2.0$ times as high as wide (Fig. 9J)

N. b. bidentatum Roewer, $1914 \times \boldsymbol{N}$. b. schmidti ssp. nov.

9. Ch-Apo high, broadly quarter moon-like, $\sim 2.4$ times as high as wide, apically sagittally shallowly bifid, Pa-Fe with evenly arched dorsal and ventral margins, Pa-Ti medio-dorsal hump conspicuous, glans terminally roundly truncated (Fig. 12A)

N. b. bidentatum Roewer, 1914

- Ch-Apo elongated oval-trapezoid, with apical pinnacle posteriorly, apically sagittally shallowly bifid, $\mathrm{Pa}-\mathrm{Fe}$ with knee-like bent dorsal margin and evenly arched ventral margin, with 3 stout spines in distal fourth, Pa-Ti with low medio-dorsal hump, and glans terminally evenly truncated (Fig. 12H)

N. b. schmidti ssp. nov.

10. Pa-Fe with knee-like bent dorsal and ventral margins ..................................................................11

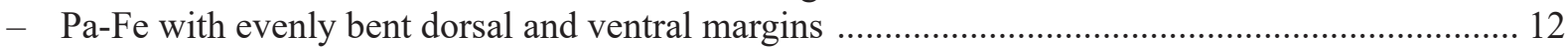

11. Ch-Apo very high, Pa-Fe with 4 stout spines in distal half (Figs 9M, 10M)

N. b. gruberi ssp. nov. $\times N$. b. schmidti ssp. nov.

- Ch-Apo high to very high, Pa-Fe with 2-3 stout spines in distal third, frontal margin bent approximately in lower quarter (Figs 9P, 10O)

N. b. schmidti ssp. nov. $\times$ N. b. sparsum Gruber \& Martens, 1968

12. Pa-Tr with high, evenly arched dorsal margin, Pa-Fe with evenly arched dorsal and ventral margins, with 4 stout, equidistant spines, Pa-Ti dorsally concave, without hump, glans isosceles triangular (Fig. 12F)

N. kozari sp. nov.

- Ch-Apo thumb-like, Pa-Fe evenly club-shaped, with 5 small denticles in distal half (Figs 9L, 10L) ................................ b. gruberi ssp. nov. $\times$ N. b. martensi ssp. nov. $\times$ N. b. schmidti ssp. nov.

13. Ch-Apo rhomboidal or apically extended

- Ch-Apo oblong with pinnacle or distinctive angle, Pa-Fe with either 2 stout spines or tubercle and pointed thorn, glans equilateral triangle (Fig. 12B) N. b. sparsum Gruber \& Martens, 1968

14. Ch-Apo apically extended

- Ch-Apo rhomboidal, Pa-Fe with either 4 subequidistantly positioned spines or pointed tubercles or long setae on low protrusions, or their combination in the distal $\mathrm{Pa}-\mathrm{Fe}$ half, or, alternatively, with $2-3$ stout thorns distally, glans isosceles triangle with width $80 \%$ of length (Fig. 12E) 
15. Ch-Apo apically extended in spire, Pa-Fe with 2 simple denticles (Figs 9N, 10N)

N. b. gruberi ssp. nov. $\times$ N. b. sparsum Gruber \& Martens, 1968

- Ch-Apo apically extended in narrow, finger-like protrusion, Pa-Fe with 5 equidistant denticles ......

N. b. gruberi ssp. nov. $\times N$. b. martensi ssp. nov.

16. Pa-Tr dorsal margin arched, Pa-Ti dorsal margin straight or negligibly concave

- Pa-Tr dorsal margin isosceles obtuse-triangular, Pa-Fe with ventral hump distally, Pa-Ti dorsal margin slightly, but conspicuously concave (Fig. 13H) N. b. sneznikensis ssp. nov.

17. Apex of Pa-Tr dorsal margin conspicuously leaned anteriorly

- Apex of Pa-Tr dorsal margin in middle

18. Frontal margin of $\mathrm{Ch}$ basal article $\sim$ straight, $\mathrm{Pa}-\mathrm{Tr}$ conspicuously step-wise broken anteriorly, $\mathrm{Pa}$ Pt relatively robust (Pa-Pt length:width $\sim 4.1: 1$ ) strait, $\mathrm{Pa}-\mathrm{Tr}$ with conspicuous step-wise indention anteriorly, Pa-Pt relatively robust (Pa-Pt length:width $\sim 4.1: 1$ ) (Fig. 13A)

N. b. bidentatum Roewer, 1914

- Frontal margin of Ch basal article convex, $\mathrm{Pa}-\mathrm{Tr}$ inconspicuously broken-bent anteriorly, $\mathrm{Pa}-\mathrm{Pt}$ relatively normally proportioned (Pa-Pt length:width 4.9:1) (Fig. 13G)

N. b. schmidti sp. nov.

19. Rec sem elongated saccular-like

- Rec sem balloon-like

20. Ch basal article length:width $\sim 2.6: 1$, Pa-Pt length:width $\sim 4.7: 1$, Rec sem of 5 irregularly-saccular alveolus (Fig. 13C) N. relictum Gruber \& Martens, 1968 stat. nov.

- Ch basal article length:width $\sim 2.9: 1$, Pa-Pt length:width $\sim 4.7: 1$, Rec sem of 5 regularly-saccular alveolus originating in bladder-like structure (Fig. 13E) N. kozari sp. nov.

21. Dorsal margin of $\mathrm{Pa}-\mathrm{Tr}$ moderately high arched, $\mathrm{Pa}-\mathrm{Fe}$ with conspicuous terminal widening ...... 22

- Dorsal margin of Pa-Tr highly arched, Pa-Fe in whole length equally widening (Fig. 13F) N. b. martensi ssp. nov.

22. Dorsal margin of Pa-Tr equally arched, Pa-Fe max width behind terminal widening vs min width $\sim 2.3: 1$ (Fig. 13B) N. b. sparsum Gruber \& Martens, 1968

- Dorsal margin of Pa-Tr with knee-like medial flexion, Pa-Fe max width behind terminal widening vs min width $\sim 1.9: 1$ (Fig. 13D) N. b. gruberi ssp. nov.

\section{Biogeography}

In Slovenia, six taxa of the $N$. $b$. complex, namely six subspecies of $N$. bidentatum exist: $N$. $b$. bidentatum, $N$. b. gruberi ssp. nov., N. b. martensi ssp. nov., $N$. b. schmidti ssp. nov., $N$. b. sparsum and $N$. b. sneznikensis ssp. nov. The distributional areas of these taxa cover most of the country (Fig. 3). Southern Austria with hitherto known three subspecies of $N$. bidentatum and one more species: $N$. b. bidentatum, $N$. $b$. schmidti ssp. nov., $N$. b. sparsum and $N$. relictum stat. nov., and north-western Croatia with four subspecies: $N$. b. gruberi ssp. nov., $N$. b. martensi ssp. nov., $N$. $b$. schmidti ssp. nov. and $N$. $b$. sparsum additionally contribute to the bulk of the $N$. $b$. complex diversity. At the time being, three species of the complex are known in Bosnia and Herzegovina: N. b. sparsum, N. pluridentatum stat. nov. and N. kozari sp. nov., two subspecies: $N$. b. gruberi ssp. nov. and $N$. $b$. schmidti ssp. nov. occur in Italy, $N$. b. sparsum and, potentially, $N$. $b$. schmidti ssp. nov. in Germany, while only $N$. b. sparsum occurs in all the other mentioned countries. In Slovenia, the main areas of the pure taxa are well defined, while the borders between the subspecies areas are not simple lines. 


\section{Discussion}

Although the process of dividing the initially single genus Nemastoma C.L. Koch, 1836 (Koch 1836), as started by Roewer (1951), into valid genera proceeded step by step (Schönhofer 2013), it was Gruber \& Martens (1968) who redefined the genus Nemastoma s. str. and began the revision of the $N$. $b$. complex. Prior to the present study, many problems regarding the $N . b$. complex were identified and discussed (Gruber 1976; Gruber \& Martens 1968; Martens 1978; Novak \& Gruber 2000; Komposch \& Gruber 2004; Komposch 2009), leading to the conclusion that there were four valid taxa within the complex, some of these with an uncertain status: $N$. b. bidentatum, $N . b$. sparsum, $N$. b. relictum and $N$. b. pluridentatum (Schönhofer 2013). Further research in Slovenia led to further taxonomic challenges, particularly with respect to the existence of new taxa and various hybrid forms, which additionally blurred the relations among the taxa, demanding a systematic and detailed reconsideration of the whole complex. So far, six subspecies of $N$. bidentatum $\mathrm{s}$. str. have been identified in Slovenia, three subspecies of $N$. bidentatum and one species in Austria and four subspecies of N. bidentatum in Croatia. Only one or two taxa sojourn in all the other mentioned countries. Accordingly, Slovenia is the most diverse country with respect to the $N$. bidentatum s. str. However, Bosnia and Herzegovina with three species within the $N$. bidentatum complex and nearly unexplored Nemastoma fauna might be the center of the $N$. bidentatum complex species diversity.

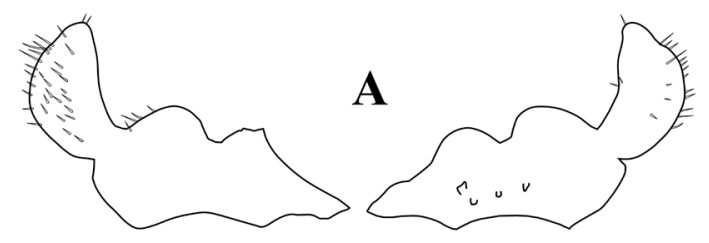

N. b. bidentatum

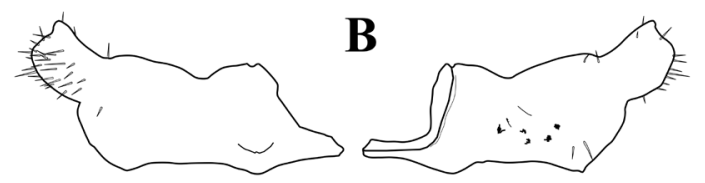

N. b. sparsum

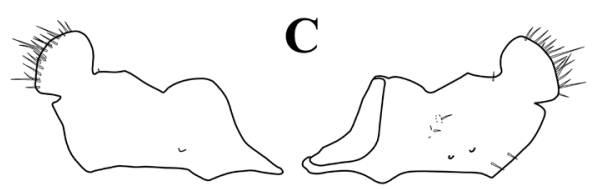

$N$. relictum stat. nov.

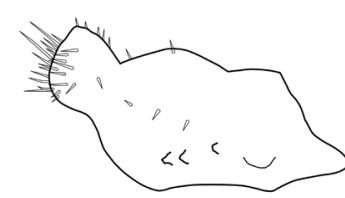

D

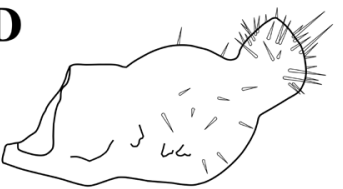

N. pluridentatum stat. nov.

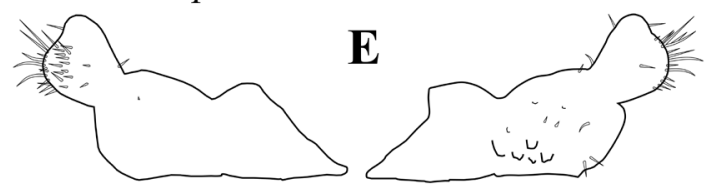

N. b. gruberi ssp. nov.

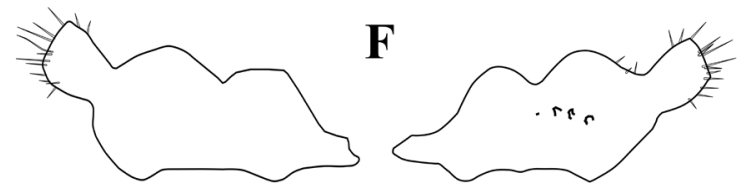

N. kozari sp. nov.

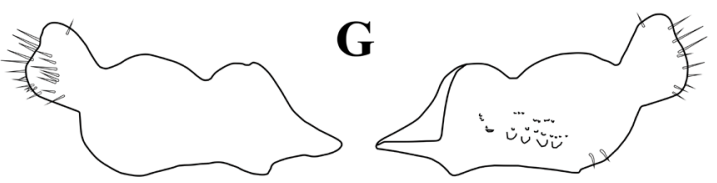

N. b. martensi ssp. nov.

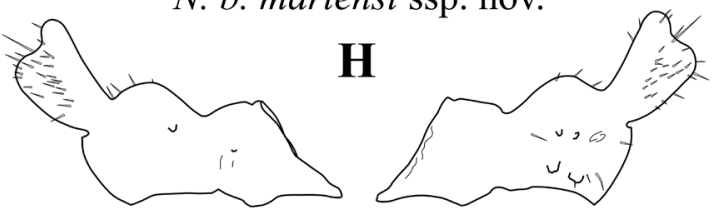

N. b. schmidti ssp. nov.

$\mathbf{I}$

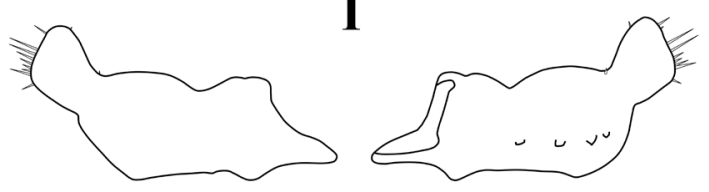

N. b. sneznikensis ssp. nov.

\section{$500 \mu \mathrm{m}$}

Fig. 4. Nemastoma bidentatum Roewer, 1914 complex, $\widehat{\jmath} \hat{\jmath}$. Ch basal articles (medial, lateral views). 
A

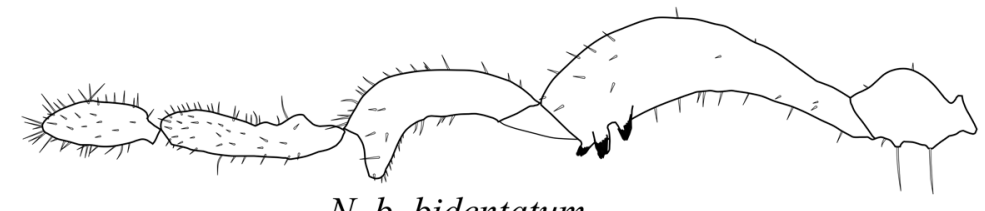

N. b. bidentatum

B

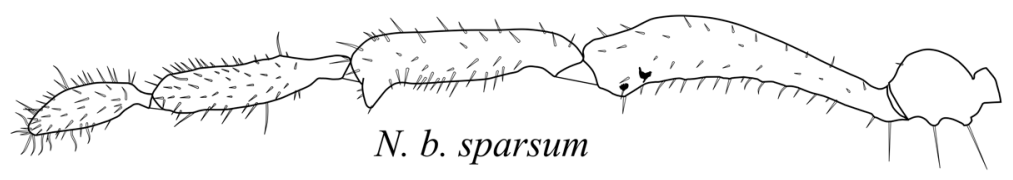

C

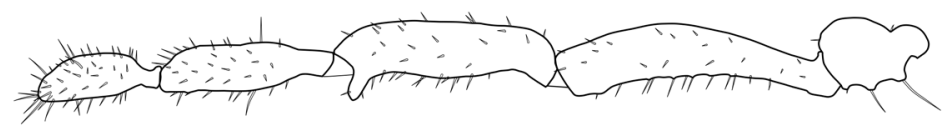

N. relictum stat. nov.

D

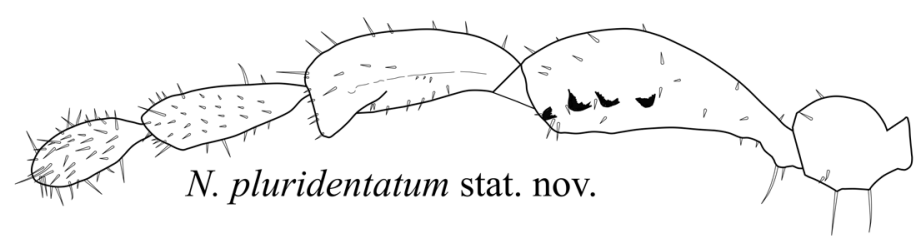

$\mathbf{E}$

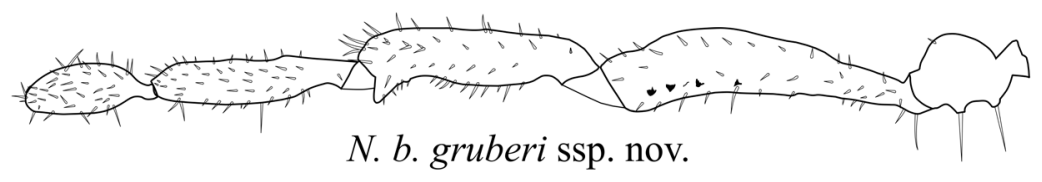

$\mathbf{F}$

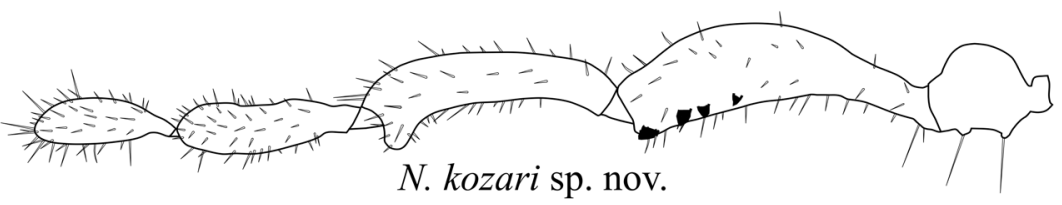

G

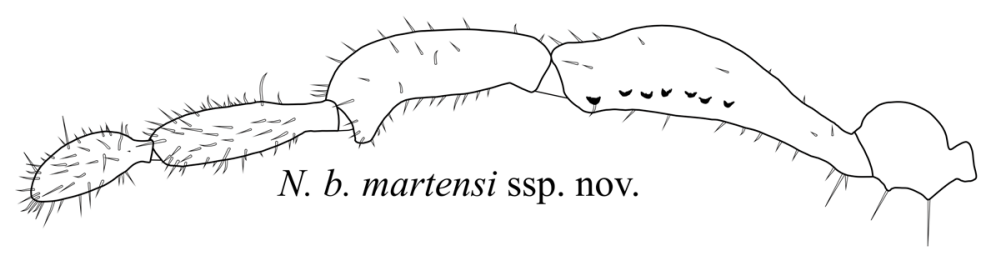

$\mathbf{H}$

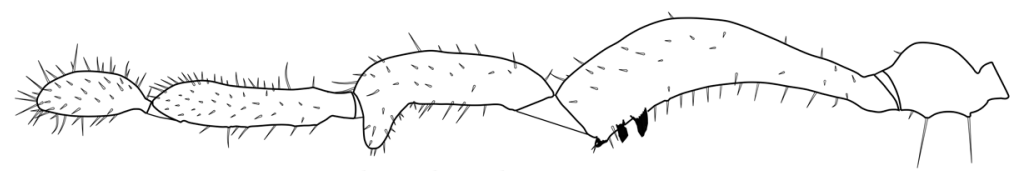

N. b. schmidti ssp. nov.

I

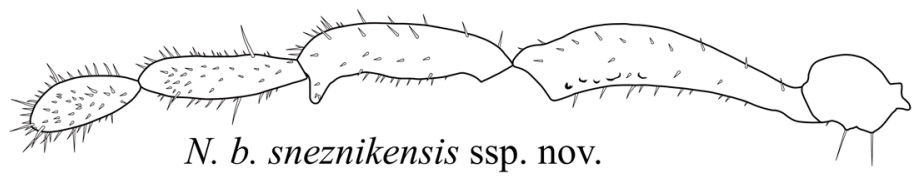

$500 \mu \mathrm{m}$

Fig. 5. Nemastoma bidentatum Roewer, 1914 complex, $\widehat{\jmath} \widehat{\jmath}$. Pa (medial view). 
A

A

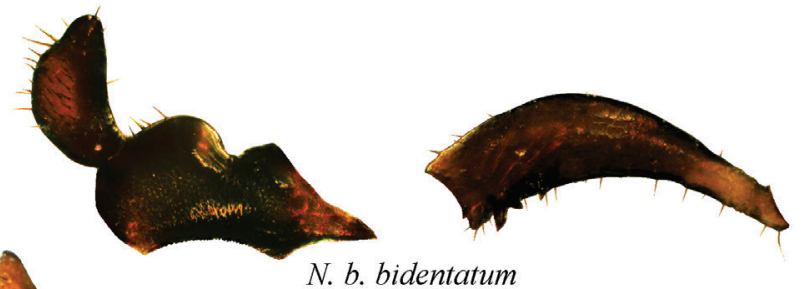

B

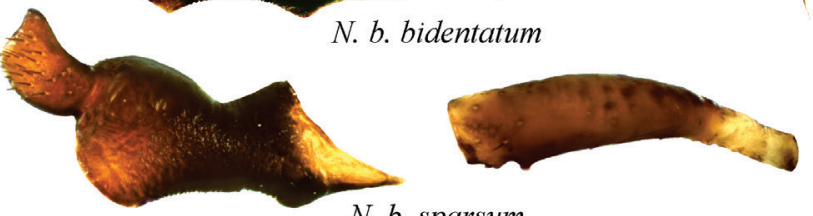

N. b. sparsum

C

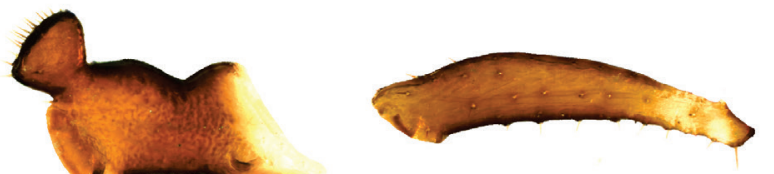

D
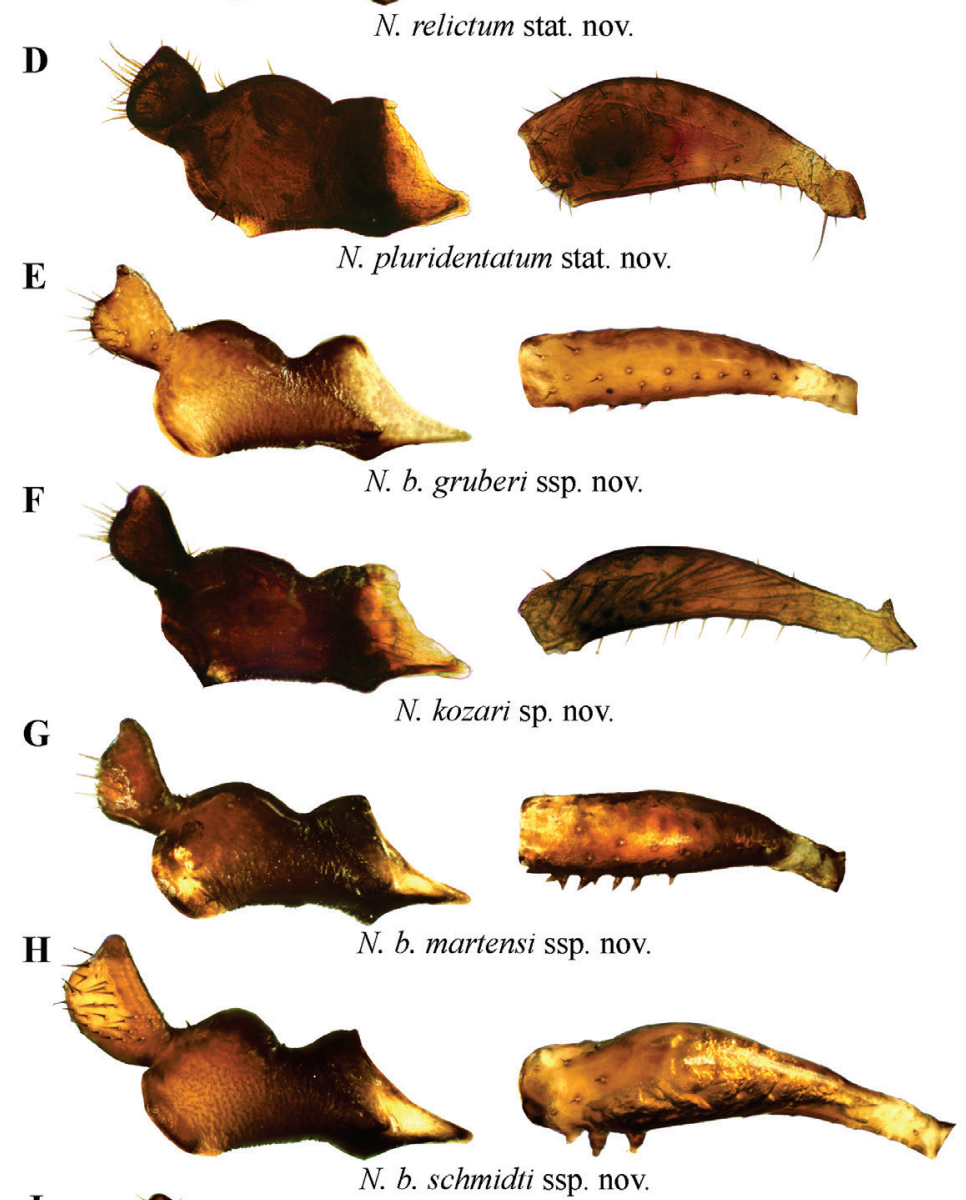

I

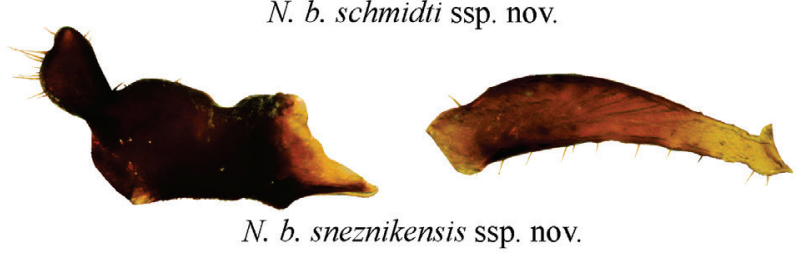

$500 \mu \mathrm{m}$

Fig. 6. Nemastoma bidentatum Roewer, 1914 complex, $\widehat{\partial} \widehat{\partial}$. Synchroscopic images of the male Ch basal article and $\mathrm{Pa}-\mathrm{Fe}$ (medial views, except $\mathrm{G}$, right figure, which is in dorso-medial view). 
The largest, western and central portion of Slovenia is occupied by $N$. $b$. schmidti ssp. nov., which meets $N$. b. bidentatum in the north, and $N$. b. martensi ssp. nov., $N$. b. gruberi ssp. nov., $N$. b. sneznikensis ssp. nov. and $N$. b. sparsum in the southwest to the northeast of the country. The central position of $N$. b. schmidti ssp. nov. in this ongoing parapatric speciation, with hybrid zones between any two subspecies in contact, is congruent with the distinct regional and geomorphological characteristics of the subspecies areas, reflecting the ecological optima of the different subspecies. On the other hand, it is probably the genetic distance between non-neighboring taxa that hinders their hybridization, e.g., in the bidentatum - schmidti ssp. nov. - sparsum taxa. While N. b. schmidti ssp. nov. interbreeds with both other taxa, no hybrids are known between $N$. b. bidentatum and $N$. b. sparsum (Gruber \& Martens 1968; Martens 1978; Komposch \& Gruber 2004; Komposch 2009). This indicates a speciation process reminiscent of that in the ring species (Irwin 2012), with a chain of interbreeding populations in contact, i.e., those acting as subspecies, and reproductively isolated populations, i.e., those acting as species to distant populations if co-occuring secondarily in syntopy.

For credible, morphologically-based identification of taxa within the $N$. $b$. complex, combined inspection of $\mathrm{Ch}, \mathrm{Pa}, \mathrm{Pe}$ and Rec sem is required; the morphology and length of the extendable ovipositor yield no useful information. This is because of the varying stability of particular characters across the taxa. The

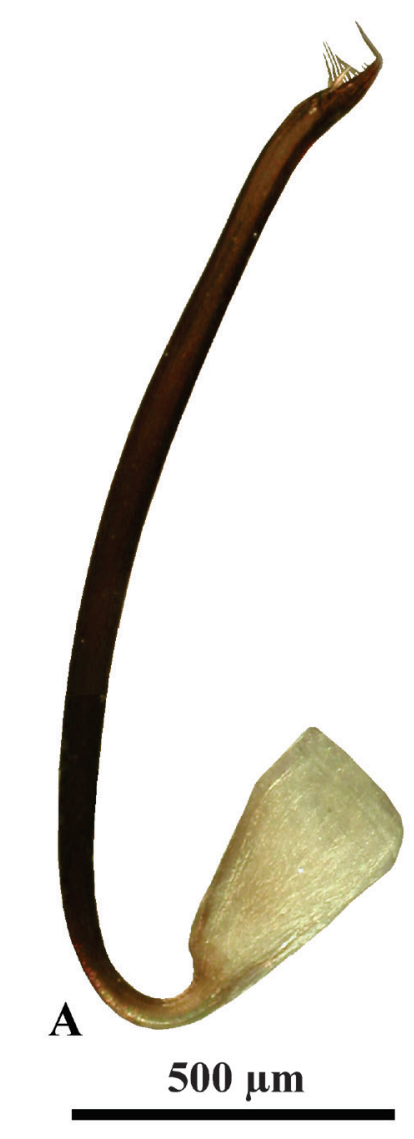

N. b. schmidti ssp. nov.
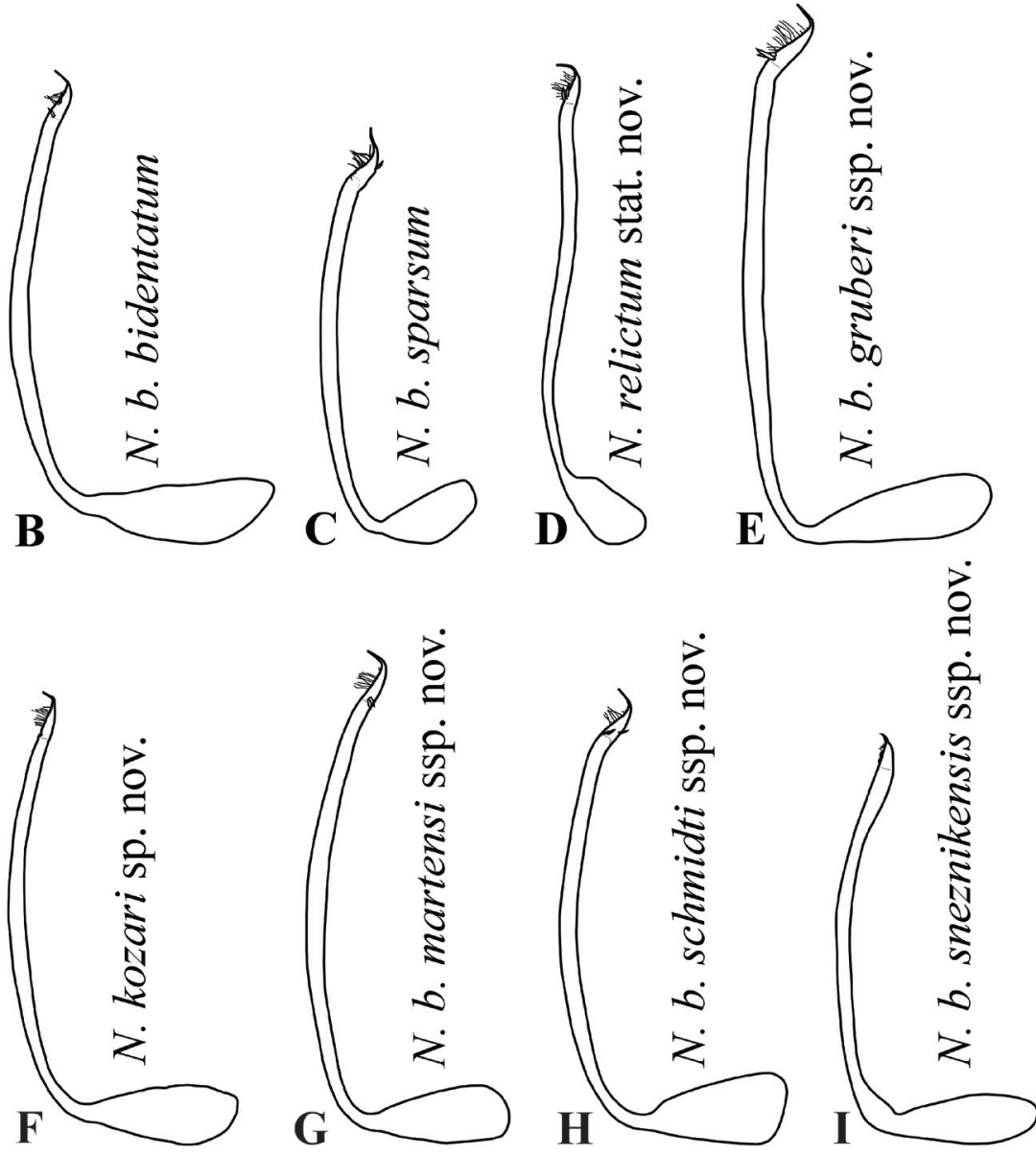

$500 \mu \mathrm{m}$

Fig. 7. Nemastoma bidentatum Roewer, 1914 complex. Penes (lateral view). Note: The penis of N. pluridentatum (Hadži, 1973) stat. nov. is unknown. 
shape of the basal $\mathrm{Ch}$ article varies considerably, while the Ch-Apo vary to a limited extent, thus being useful for identification. Only $N$. b. bidentatum and $N$. $b$. schmidti ssp. nov. can be identified on the basis of the Ch-Apo shape alone. The Pa morphology is much less stable. Gruber \& Martens (1968) identified morphological variation in the male Fe-Pa in $N$. $b$. bidentatum, which has two or three distal medio-ventral spines, one of which may be bifid. In N. b. martensi ssp. nov., the number and shape of denticles vary from 5 to11, and from pointed denticles alone to irregularly shaped, blunt tubercles instead of denticles in a row. Instead, $N$. $b$. sneznikensis ssp. nov. has a row of minute, irregularly shaped tubercles. Such moderate variation is usual in all subspecies, but in $N$. b. gruberi ssp. nov. it is excessive. In this subspecies, the $\mathrm{Pa}-\mathrm{Fe}$ armament varies from four equidistant, relatively thin medio-ventral spines of subequal sizes in individuals from central-eastern Slovenia, to very small spines or ones diminished to a tubercle with a seta in the corresponding position in the south, or 2 to 3 large, stout thorns in the southwest. Furthermore, the presence of a hump on the Pa-Ti indicates either N. bidentaum $b$. or $N$. $b$. schmidti ssp. nov. As for the genital characters, the whole Pe size and shape, including the glans shape, are rather unsuitable for

A

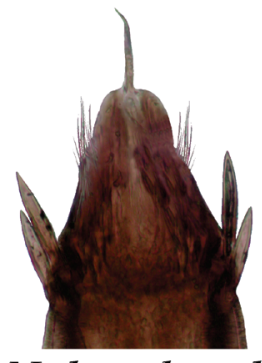

N. b. schmidti ssp. nov.

D
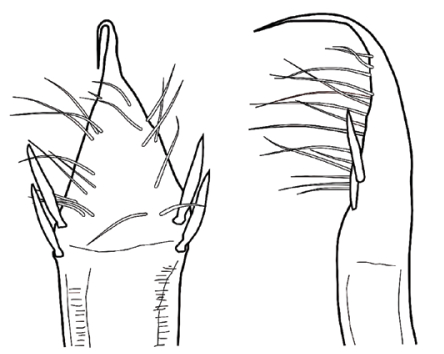

N. relictum stat. nov.

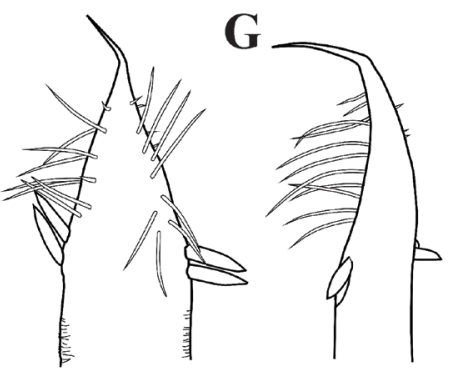

B

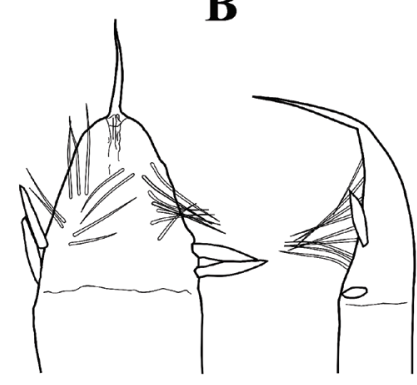

N. b. bidentatum

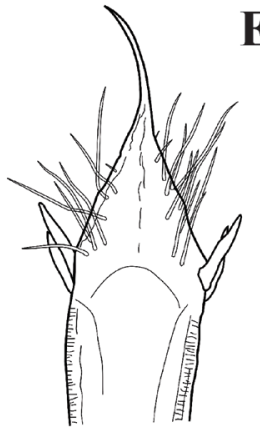

$\mathbf{E}$

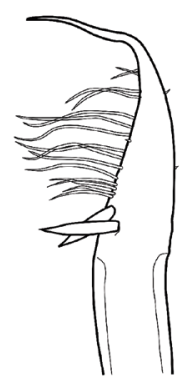

N. b. gruberi ssp. nov.

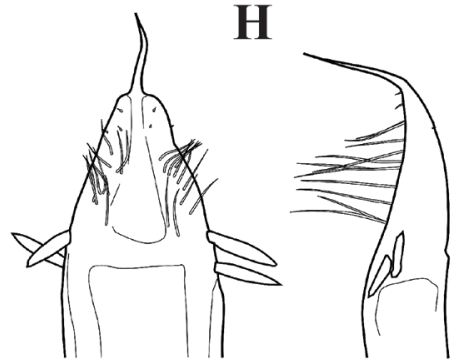

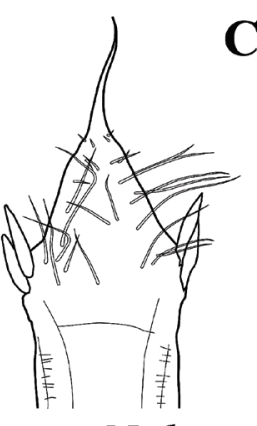

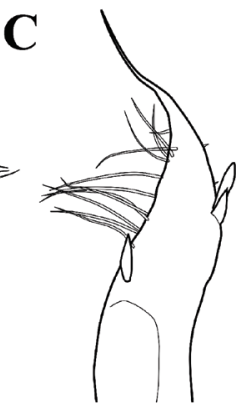

N. b. sparsum

F

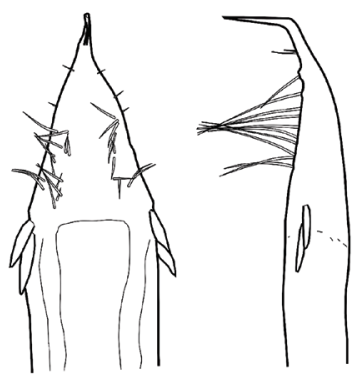

N. kozari sp. nov.

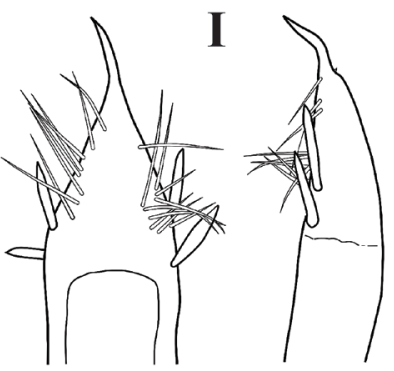

N. b. martensi ssp. nov. N. b. schmidti ssp. nov. N. b. sneznikensis ssp. nov. $100 \mu \mathrm{m}$

Fig. 8. Nemastoma bidentatum Roewer, 1914 complex. Glans (dorsal and lateral views). Note: The penis of N. pluridentatum (Hadži, 1973) stat. nov. is unknown. 
identification, with the exception of the truncated tip in $N$. b. bidentatum and $N$. $b$. schmidti ssp. nov. On the other hand, the Rec sem in $N$. relictum stat. nov. and $N$. kozari sp. nov. alone allow credible identification. Otherwise, the genital characters in combination with $\mathrm{Ch}$ and $\mathrm{Pa}$ characters together allow credible identification in males, and the $\mathrm{Ch}, \mathrm{Pa}$ and $\mathrm{Rec}$ sem in females. In previous studies, females have routinely been ascribed to a taxon in accordance with males identified in the sample (Gruber pers. com.).

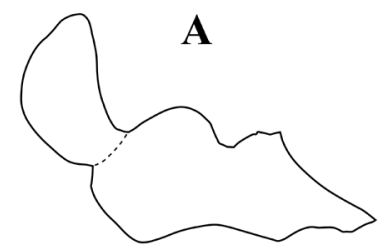

N. b. bidentatum

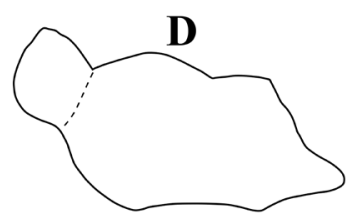

$N$. pluridentatum stat. nov.

G

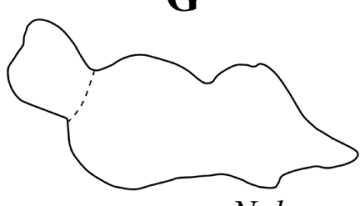

N. b. martensi ssp. nov.

$\mathbf{H}$

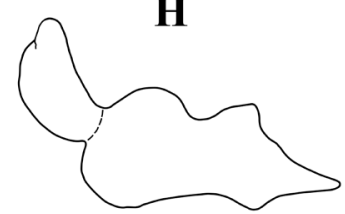

N. b. schmidti ssp. nov.

K

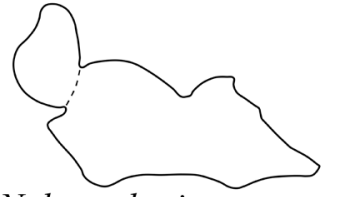

N. b. gruberi ssp. nov. $\mathrm{x}$

$N$. b. martensi ssp. nov.

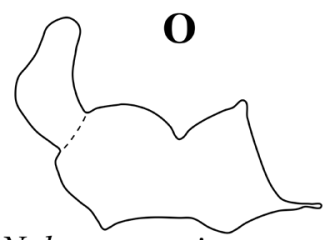

N. b. martensi ssp. nov. $\mathrm{x}$

N. b. schmidti ssp. nov.
B

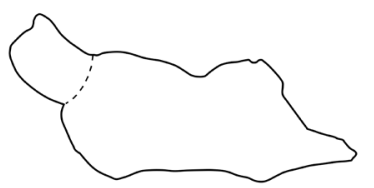

N. b. sparsum

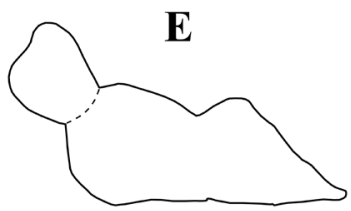

N. b. gruberi ssp. nov.

G

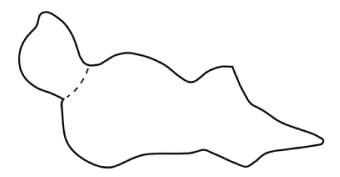

I

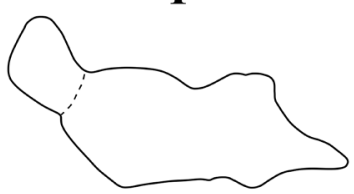

N. b. sneznikensis ssp. nov.

$\mathbf{L}$

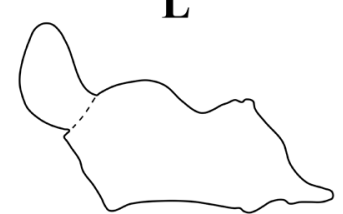

N. b. gruberi ssp. nov. $\mathrm{x}$

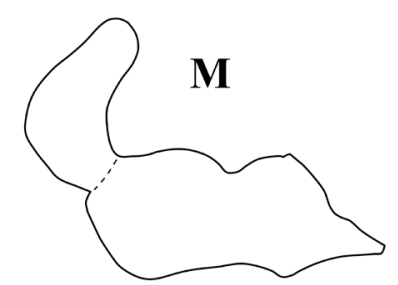

N. b. gruberi ssp. nov.

$\mathrm{x} N$. b. schmidti ssp. nov.

N. b. martensi ssp. nov.

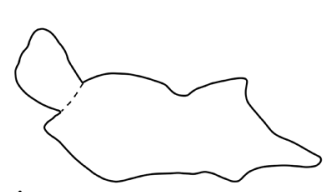

B

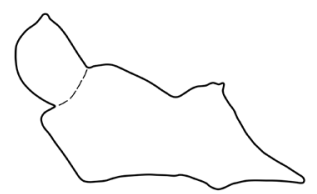

$\mathbf{E}$

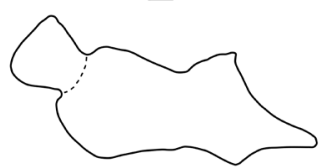

H

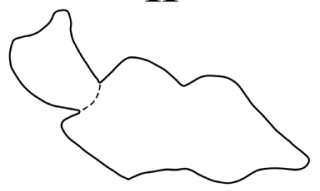

N. b. schmidti ssp. nov.
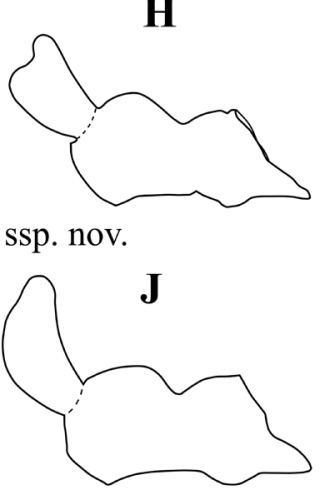

$N . b$. bidentatum $\times N . b$. schmidti ssp. nov.

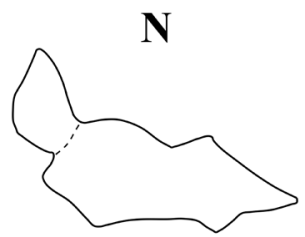

N. b. gruberi ssp. nov.

$\mathrm{x} N$. b. sparsum
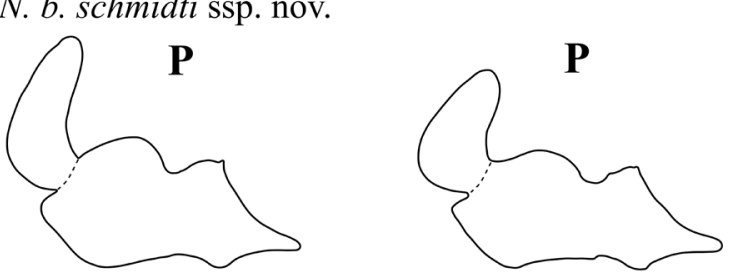

$N$. b. schmidti ssp. nov. x N. b. sparsum

\section{$500 \mu \mathrm{m}$}

Fig. 9. Nemastoma bidentatum Roewer, 1914 complex, $\widehat{\partial} \widehat{\partial}$. Variation of the Ch basal article (medial view) in species, subspecies and hybrids. 
However, the Rec sem, and the $\mathrm{Ch}$ basal article, $\mathrm{Pa}-\mathrm{Tr}$ and $\mathrm{Pa}-\mathrm{Fe}$ shapes are diagnostic to a varying extent, but always in combination. Males of pure subspecies are easy to identify, while in few cases, identification of females with Rec sem consisting of balloon-like vesicles alone is not certain.

A

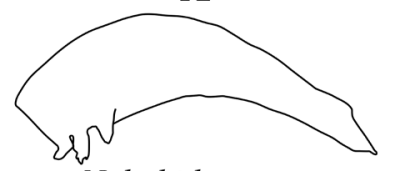

N. b. bidentatum

D

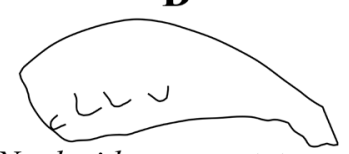

$N$. pluridentatum stat. nov.

$\mathbf{E}$

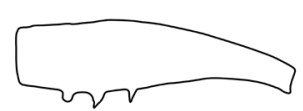

N. b. gruberi ssp. nov.

G

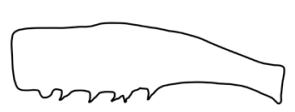

N. b. martensi ssp. nov.

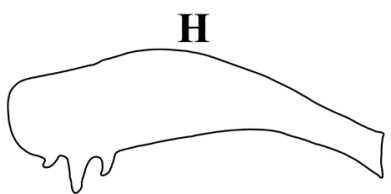

N. b. schmidti ssp. nov.

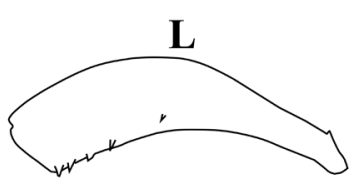

$N$. b. gruberi ssp. nov. $\mathrm{x}$

N. b. martensi ssp. nov. $\mathrm{x}$

$N$. b. schmidti ssp. nov.

$\mathbf{O}$

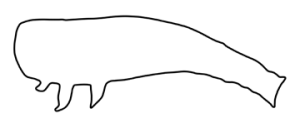

B

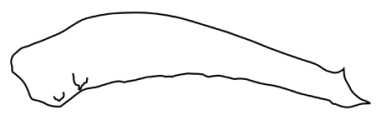

$\mathbf{E}$

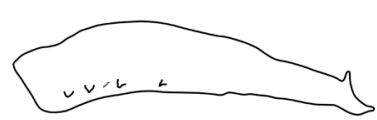

$\mathbf{E}$

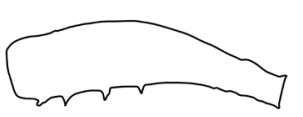

G

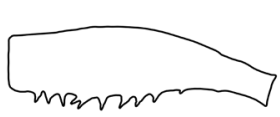

ssp. nov.

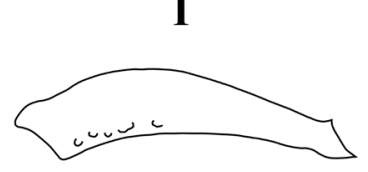

N. b. sneznikensis ssp. nov.

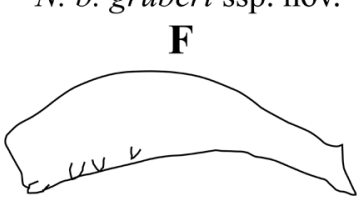

N. kozari sp. nov.

$\mathbf{H}$

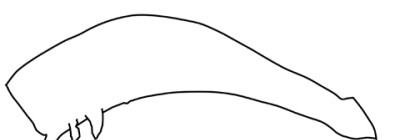

N. b. schmidti ssp. nov.

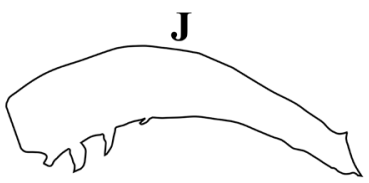

$N$. b. bidentatum $\times$ N. $b$. schmidti ssp. nov.
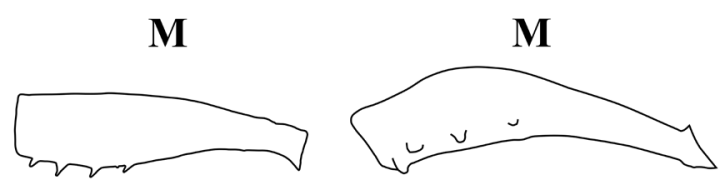

$N$. b. gruberi ssp. nov. x N. b. schmidti ssp. nov.

$\mathbf{O}$

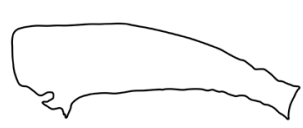

N. b. schmidti ssp. nov. x N. b. sparsum
C

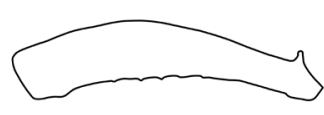

$N$. relictum stat. nov.

E
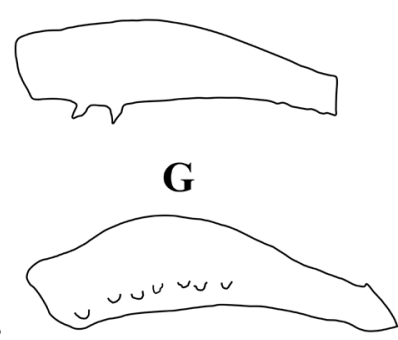

N. b. martensi ssp. nov.

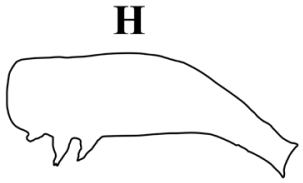

$\mathbf{K}$

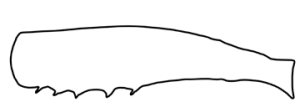

N. b. gruberi ssp. nov. $\mathrm{x}$ N. b. martensi ssp. nov.

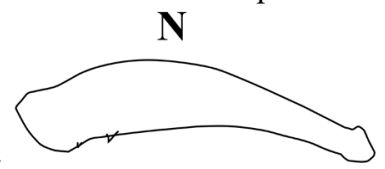

N. b. gruberi ssp. nov. $\mathrm{x}$

N. b. sparsum

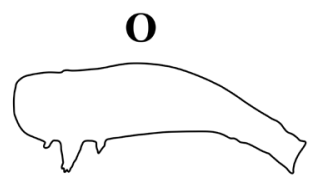

\section{$500 \mu \mathrm{m}$}

Fig. 10. Nemastoma bidentatum Roewer, 1914 complex, $\widehat{\partial}$. Variation of the Pa-Fe (A-B first image, C, D-E first image, F-G first image; H-J, L-M right, N-O: lateral view; the others in dorso-medial view to show the armament). 
We applied the subspecies concept to all relatively uniform populations that exhibit constant differences with respect to other such adjacent populations while interbreeding with them. The standard quantitative level for defining a subspecies is the $75 \%$ rule (Patten \& Unitt 2002), which states that a subspecies is valid if at least $75 \%$ of a population lies outside $99 \%$ of the range of the overlapping population for a given character. However, there is disagreement about the $75 \%$ threshold and the number of characters that should be used when comparing populations (Patten \& Unitt 2002). In subspecies of N. bidentatum, this rule can easily be confirmed for all the subspecies, except the polymorphic $N$. $b$. gruberi ssp. nov. All these taxa fulfill the Braby et al. (2012) recommendation for subspecies status. This states that under the unified species lineage, the subspecies are extant groups representing partially isolated lineages of a species that are allopatric, phenotypically distinct, and have one fixed, diagnosable character state. Beside pure subspecies, most typical hybrid males can be easily identified, while hybrids with prevailing characters of one subspecies, and hybrid females are poorly identifiable. In most subspecies combinations, typical hybrid males are characterized by their elongated Ch-Apo, mostly without an apical pinnacle, and usually with a more or less expressed, knee-like flexion of the frontal margin. In contrast, $N$. b. gruberi ssp. nov. $\times N$. $b$. sparsum males have a low, quarter moon-like Ch-Apo, which can only be evaluated in combination with the Pa characters. In any doubtful cases, biogeographical data allow a credible check.

The status of the four species is argued according to combined criteria. The fist described $N$. bidentatum is a complex of six subspecies; however, the nominal $N$. b. bidentatum is markedly distinctive from all other taxa within the complex. We gave the species status to $N$. relictum stat. nov. because it has Rec sem with five elongated, sack-like vesicles, which makes it distinctly different from all the subspecies of $N$. bidentatum, in which the Rec sem consists of 12 to 18 balloon-like vesicles. Besides, it lives syntopically with another taxon of the $N . b$. complex, without hybridization. In the case of $N$. pluridentatum stat. nov., we took into account that this taxon shows explicit morphological difference from any other taxon of the $N$. $b$. complex, including the recently discovered $N$. kozari sp. nov. inhabiting the same mountain.

A

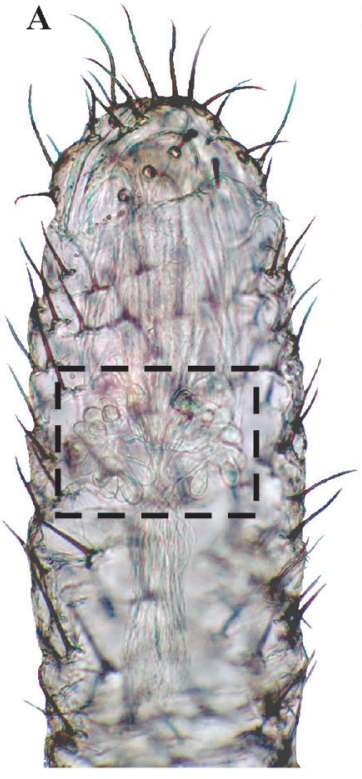

$100 \mu \mathrm{m}$

N. b. sparsum

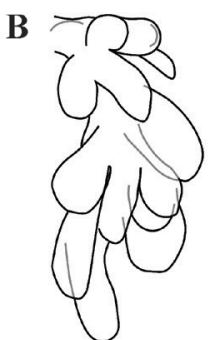

N. b. bidentatum

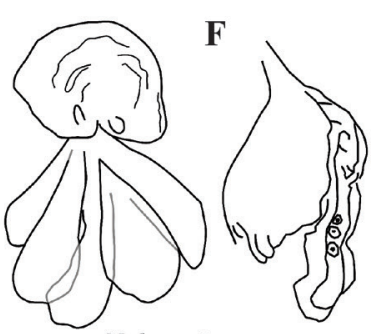

N. kozari sp. nov.

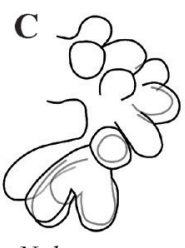

N. b. sparsum
D

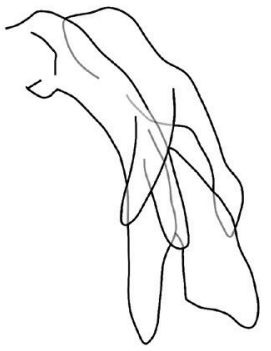

$N$. relictum stat. nov.

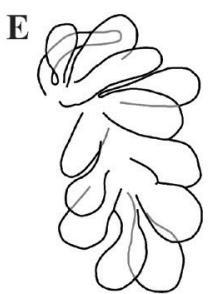

N. b. gruberi ssp. nov.

$\mathbf{H}$

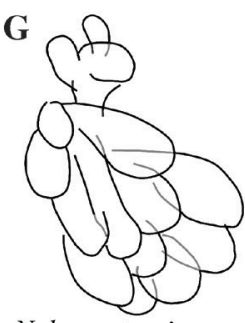

N. b. martensi ssp. no
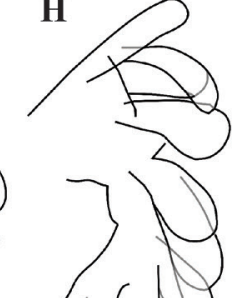

N. b. sneznikensis ssp. nov.

$50 \mu \mathrm{m}$

Fig. 11. Nemastoma bidentatum Roewer, 1914 complex, $\circ+$; Rec sem (lateral view, right portion, except F left figure, which is in dorsal view). A. The frame encloses the ovipositor portion with Rec sem. Note: Female N. pluridentatum (Hadži, 1973) stat. nov. is unknown. 
A

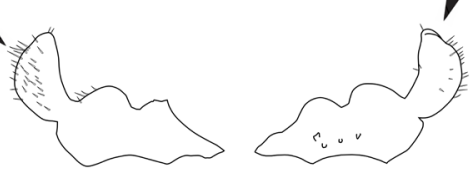

B

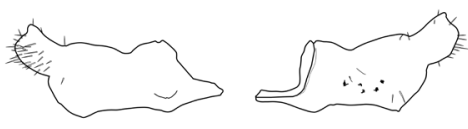

C

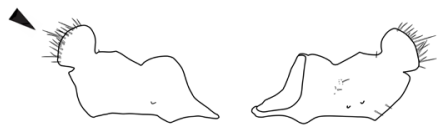

D

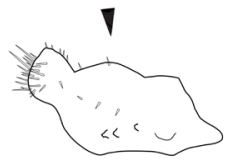

$\mathbf{E}$
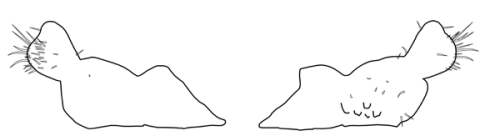

F

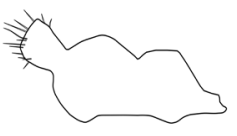

G

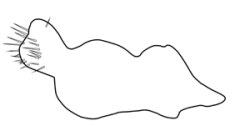

H
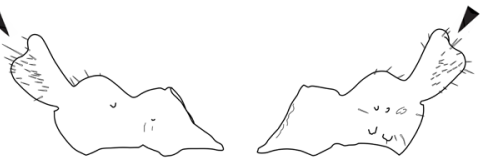

I
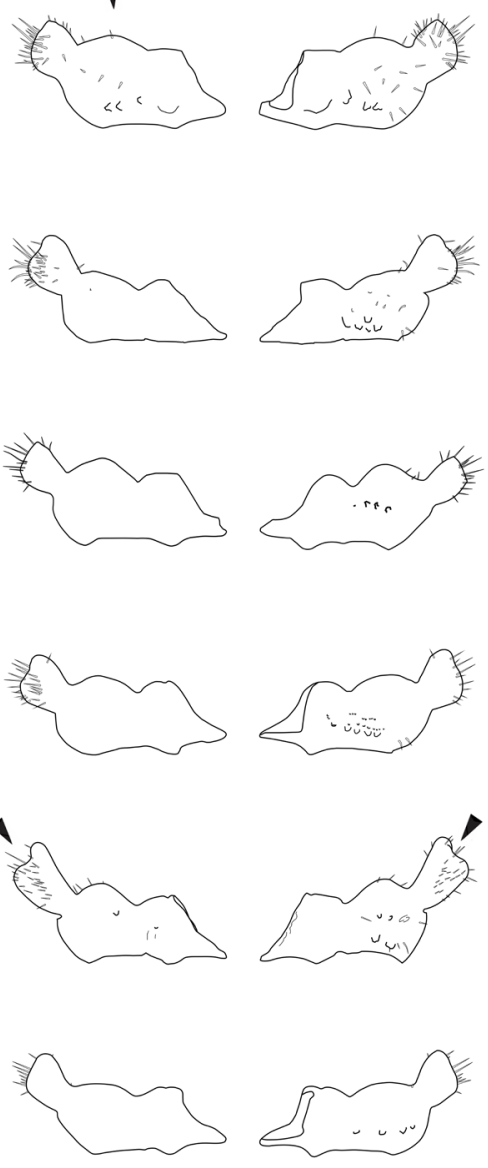
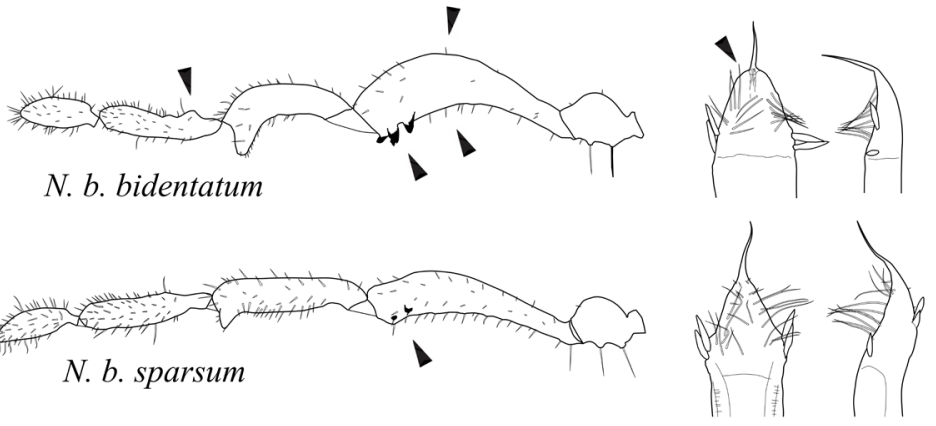

N. b. sparsum

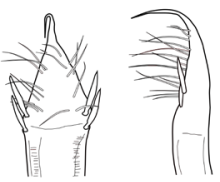

N. relictum stat. nov.

N. pluridentatum stat. nov.
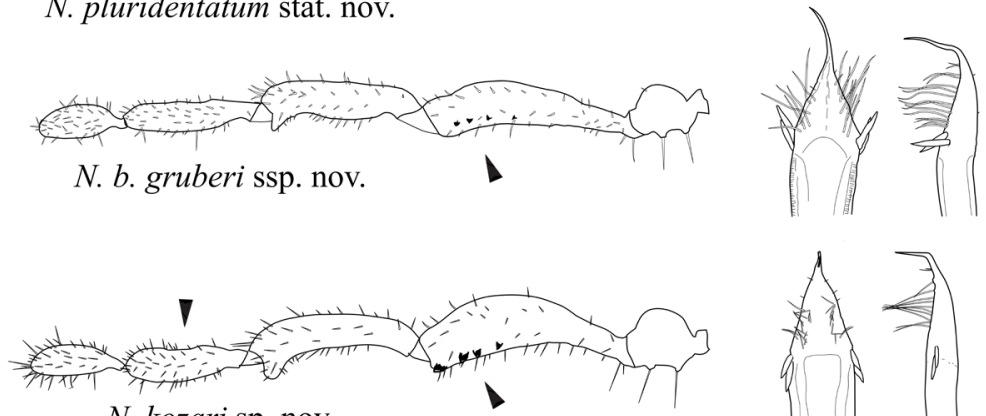

N. kozari sp. nov.
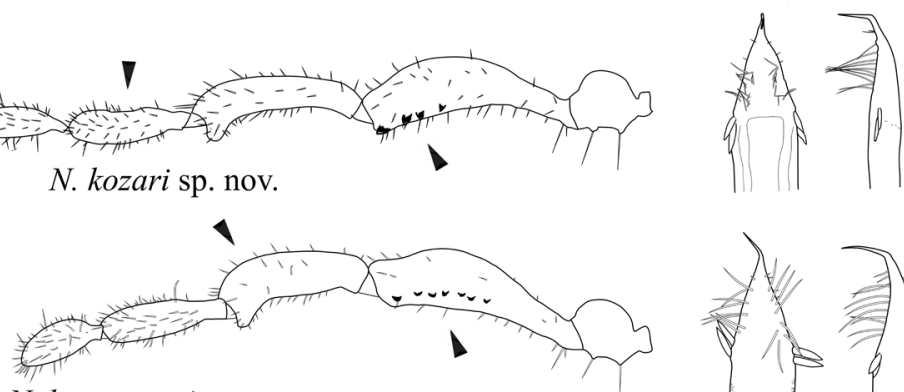

N. b. martensi ssp. nov.
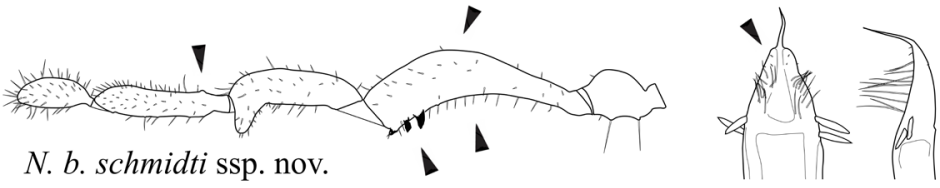

N. b. sneznikensis ssp. nov.

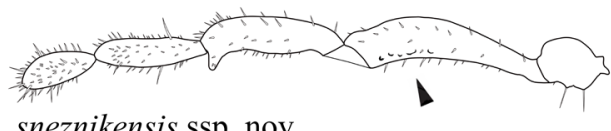

$500 \mu \mathrm{m}$

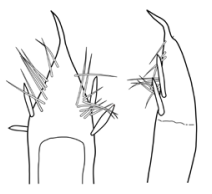

$100 \mu \mathrm{m}$

Fig. 12. Nemastoma bidentatum Roewer, 1914 complex, $\widehat{\partial} \hat{\partial}$. From left to right: Ch basal article (medial, lateral views), $\mathrm{Pa}$ (medial view) and Pe tip (dorsal, lateral views). Arrows indicate the most identifying characters. Note: Pe of N. pluridentatum (Hadži, 1973) stat. nov. is unknown. 
A

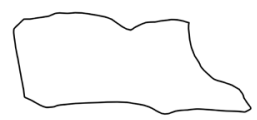

B

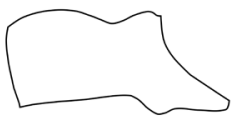

C

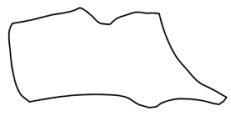

D

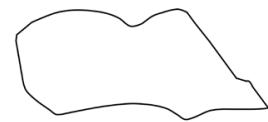

$\mathbf{E}$

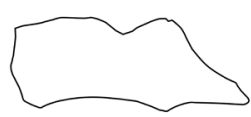

$\mathbf{F}$

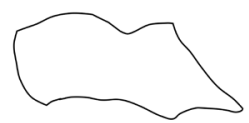

G

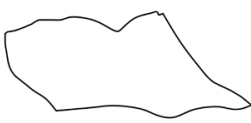

$\mathbf{H}$

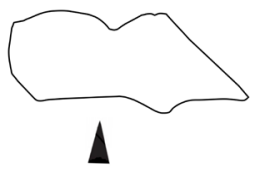

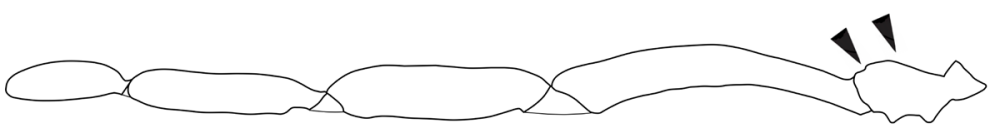

N. b. bidentatum
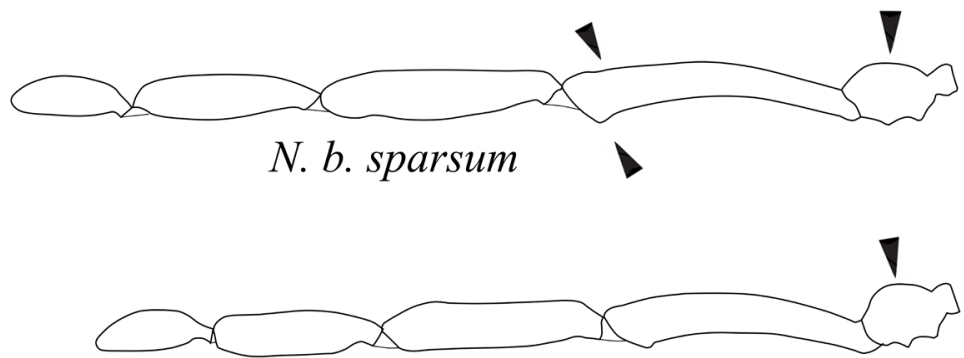

N. relictum stat. nov.

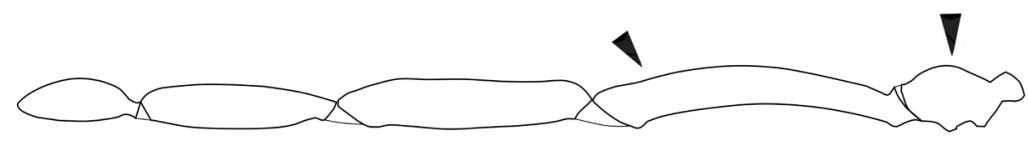

N. b. gruberi ssp. nov.

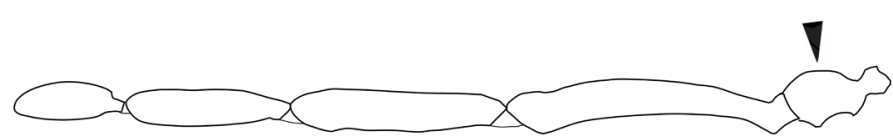

N. kozari sp. nov.

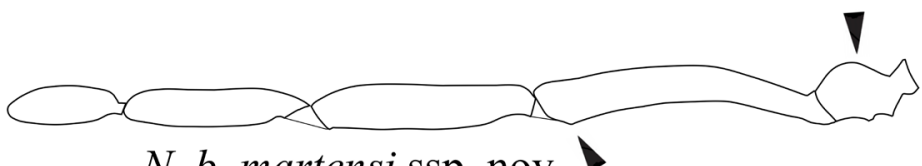

N. b. martensi ssp. nov.

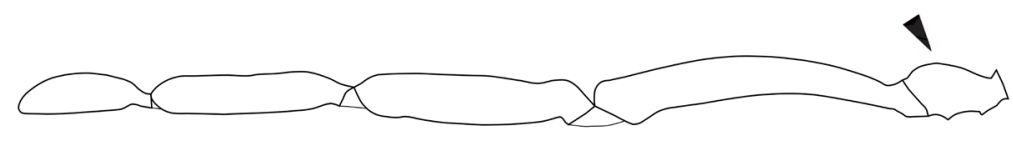

N. b. schmidti ssp. nov.

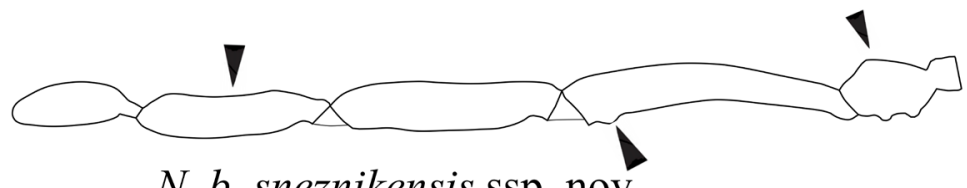

N. b. sneznikensis ssp. nov.

\section{$\mathbf{5 0 0} \boldsymbol{\mu m}$}
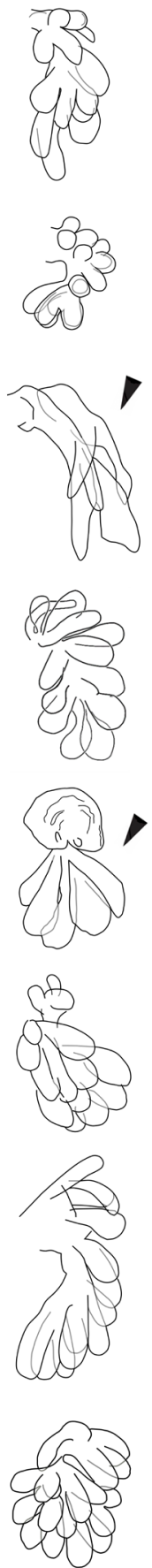

$\mathbf{5 0} \mu \mathrm{m}$

Fig. 13. Nemastoma bidentatum Roewer, 1914 complex, $q$ ㅇ․ From left to right: Silhouettes of the $\mathrm{Ch}$ basal article, $\mathrm{Pa}$ (both medial view) and Rec sem. Arrows indicate the most identifying characters. Note: female N. pluridentatum (Hadži, 1973) stat. nov. is unknown. 
Nemastoma relictum stat. nov., $N$. pluridentatum stat. nov. and $N$. $b$. sneznikensis ssp. nov. are relic taxa inhabiting montane to alpine habitats.

All the morphological findings in this study are supported by chemotaxonomic investigation of the scent gland profiles of the taxa under consideration and by genetics (to be published separately), but these are currently lacking for $N$. $b$. sneznikensis ssp. nov. due to its sparseness. Further taxa within the $N$. b. complex are expected in the Balkans, especially in the Dinarides.

A clear-cut contact zone between the subspecies of $N$. bidentatum has not been found. Sharp boundaries, such as referred to by Gruber \& Martens (1968) and Martens (1978), may occur due to a barrier habitat between the two populations (cf. Monahan et al. 2012), such as a wide brook with pebble, sand or clay banks, representing an unsuitable habitat for both subspecies. Otherwise, the contact zones between areas of vicariant subspecies follow the geomorphological variation, or there are narrow or wider hybrid zones in between. Individuals of adjacent subspecies that usually live vicariantly in the contact zone, live syntopically in a contact area of various habitats, e.g., a riparian and an adjacent steep dry forest, or Nardion strictae and Hyperico grisebachii-Pinetum mugo associations, which might comprise zones only a few meters wide. In most localities in the hybrid zones, hybrids of varying intermediate stages are present, and in some places they live in syntopy with individuals showing pure characters of one or both neighboring subspecies. Most interbreeding zones are a few to $20 \mathrm{~km}$ wide, while the overlapping area of $N$. $b$. schmidti ssp. nov. and $N$. b. sparsum is over $100 \mathrm{~km}$ wide in eastern Slovenia. Taking into account various environmental conditions related to the subspecies requirements in the main distribution areas, such flexible borders could be understood.

Field notes on microhabitats inhabited by individual taxa of $N$. b. complex allowed us to conclude that all these taxa - with the exception of $N$. pluridentatum with unknown habitat preferences - are specialized for living in loose litter, deep cushion moss and similar loose microhabitats in riparian, deciduous and pine forests, various scrublands, orchards and high-turf grasslands, and maybe a humus-rich, shallow subterranean habitat (SSH), originally named Milieu Souterrain Superficiel (MSS) (Mammola et al. 2016; Halse 2018). Typically, the loose, organic substrate is at least a few centimeters deep, moist, not dry or wet, and with at least a small amount of mull humus. In humid, calm conditions, individuals move on the surface and sometimes climb mushrooms, rotten wood and similar objects. However, individuals are very scarce or lacking at sites with compact litter due to abundant precipitation or the formation of macroaggregates by fungal activity (Coleman et al. 2018). This is true for any habitat type, including natural and moderately managed habitats. Thus, the taxa of $N$. b. complex are indicators of loose organic substrate producing humus soils, which indicates a type of pedological process with rich organic strata rather than a specific plant community.

We consider the $N$. $b$. complex a perfect opportunity to study speciation in Opiliones in general, and Dyspnoi in particular. In the following contributions we shall present chemotaxonomy based on the scent gland profiles and the genetics of the taxa under study. Despite the taxonomic complexity of the $N$. bidentatum group we conclude that morphological approach, based on detailed morphological study and suported by another taxonomic approaches, allows credible identification, which might importantly alleviate current faunistic and ecological investigations.

\section{Acknowledgements}

Many colleagues, friends and students provided the material and made this study possible: Andreini, Barbara Bertoncelj, I. Bertoncelj, Urška Bertoncelj, Savo Brelih, Matjaž Černila, Božo Drovenik, Aleš Gergeli, Vesna Germovšek, Fulvio Gasparo, Gianluca Governatori, Jürgen Gruber (JG), Jovan Hadži (JH), Bogdan Horvat, Hribernik, Simona Hudrap, Petra Igerc, Franc Janžekovič, Andrej Kapla, Stanko Karaman, Aleksander Kocuvan, G. Kolar, Christian Komposch, Koren, Dunja Kos, Ivan Kos, Ildikó 
Kovač, V. Kovačič, Senad Kozar, Peter Kozel (PK), Tjaša Kozel, Drago Kuhar, Matjaž Kuntner, Valika Kuštor, Ivan Leitinger, Saška Lipovšek (SL), Jochen Martens (JM), Mencinger, Mojca Mlakar, K. Musar, Tone Novak (TN), Boštjan Ogrizek, B. Oven, Roman Ozimec, Majda Pavlin, Peričnik, Alja Pirnat, Jan Podlesnik, Slavko Polak, Anton Polenec, Franc Potočnik, R. Potočnik, M. Potokar, Janez Ravljen, Sonja Samu, Axel Schönhofer (ASc), Albin Seliškar, Ignac Sivec, Ljuba Slana Novak (LSN), Maja Slana Novak, Jože Staudacher, Rudolf Sturany, Mojmir Štangelj, Tina Tkavc, Tomi Trilar, Peter Trontelj, H. Urbas, A. Valenčak, Milena Varžič, France Velkovrh, M. Vončina, D. Vrček, Maja Zagmajster, Ana Ziherl, Nataša Žmaher and A. Žunič. We are indebted to Michelle Gadpaille for valuable improvement of the language. We are deeply grateful to two anonymous referees for their corrections and many insightful comments and suggestions for improvement of the manuscript.

\section{Competing interests}

The authors declare no competing interests.

\section{Funding}

This study was partly supported by the Slovenian Research Agency within the Biodiversity Research Program (Grant No. P1-0078), by the Project "Development of research infrastructure for the International competitiveness of the Slovenian RRI space - RI-SI-LifeWatch" (the operation is co-financed by the Republic of Slovenia, Ministry of Education, Science and Sport and the European Union from the European Regional Development Fund) and by the Austrian Science Fund (FWF), project number P29699-B25.

\section{References}

Babalean A.F. 2005. General overview of the opilionid fauna (Arachnida, Opiliones) in Romania. Scientific Annals of ,, Alexandru Ioan Cuza” University of Iasi. (New Series), Section 1. Animal Biology 51: 47-54.

Blick T. \& Komposch C. 2004. Checkliste der Weberknechte Mittel- und Nordeuropas. Checklist of the harvestmen of Central and Northern Europe (Arachnida: Opiliones). Version 27. December 2004. Available from https://arages.de/fileadmin/Pdf/checklist2004_opiliones.pdf [accessed 25 Feb. 2021].

Braby M.F., Eastwood R. \& Murray R. 2012. The subspecies concept in butterflies: has its application in taxonomy and conservation biology outlived its usefulness? Biological Journal of the Linnean Society 106: 699-716. https://doi.org/10.1111/j.1095-8312.2012.01909.x

Coleman D.C., Callaham Jr. M.A. \& Crossley Jr. D.A. 2018. Fundamentals of Soil Ecology. $3^{\text {rd }}$ Ed. Elsevier Academic Press.

Ćurčić B.P.M., Karaman I.M. \& Tomić V.T. 1999. On some new and little known epigean harvestmen (Opiliones, Arachnida) from west Serbia, Yugoslavia. Arches of Biological Sciences, Belgrade 51 (4): $61-62$.

De Queiroz K. 2007. Species concepts and species delimitation. Systematic Biology 56: 879-886. https://doi.org/10.1080/10635150701701083

Fernández R., Sharma P.P., Tourinho A.L. \& Giribet G. 2017. The Opiliones tree of life: shedding light on harvestmen relationships through transcriptomics. Proceedings of the Royal Society B 284: e20162340. https://doi.org/10.1098/rspb.2016.2340

Giribet G. \& Sharma P.P. 2015. Evolutionary biology of harvestmen (Arachnida, Opiliones). Annual Review in Entomology 60: 157-175. https://doi.org/10.1146/annurev-ento-010814-021028

Gruber J. 1976. Ergebnisse zoologischer Sammelreisen in der Türkei: Zwei neue Nemastomatidenarten mit Stridulationsorganen, nebst Anmerkungen zur systematischen Gliederung der Familie (Opiliones, Arachnida). Annalen des Naturhistorischen Museums in Wien 80: 781-801. 
Gruber J. 2007. Dyspnoi - Historical systematic synopsis and family chapters. In: Pinto-da-Rocha R., Machado G. \& Giribet G. (eds) Harvestmen: the Biology of Opiliones: 131-159. Harvard University Press, Cambridge MA.

Gruber J. \& Martens J. 1968. Morphologie, Systematik und Ökologie der Gattung Nemastoma C.L. Koch (s. str.) (Opiliones, Nemastomatidae). Senckenbergiana Biologica 49 (2): 137-172.

Hadži J. 1927. Opilioni Schmidtove zbirke. Glasnik Muzejskega Društva za Slovenijo 7-8: 1-41 + VIII.

Hadži J. 1928. Beitrag zur Kenntnis der Opilioniden-Fauna von Slowenien (Jugoslawien). Zoologischer Anzeiger 77 (1-2): 5-19.

Hadži J. 1931. Opilioni Triglavskoga masiva. Prirodoslovne razprave 1: 107-154.

Hadži J. 1973a. Novi taksoni suhih južin (Opilionidea) v Jugoslaviji. Razprave SAZU, IV. razred 16 (1): $1-120$.

Hadži J. 1973b. Opilionidea. In: Hadži J., Lorković Z., Horvatić S., Bole J. \& Martinčič A. (Consilium JAZU, eds) Catalogus Faunae Jugoslaviae III/4: 1-24. SAZU, Ljubljana.

Haig M., Beever E.A., Chambers S.M., Draheuim H.M., Dugger B.D., Dunham S., Elliot-Smith E., Fontaine J.B., Kesler D.C., Knaus B.J., Lopes I.F., Loschl P., Mullins T.D. \& Sheffield L.M. 2006. Taxonomic considerations in listing subspecies under the U.S. Endangered Species Act. Conservation Biology 20 (6): 1584-1594. https://doi.org/10.1111/j.1523-1739.2006.00530.x

Halse S. 2018. Research in calcretes and other deep subterranean habitats outside caves. In: Moldovan O.T., Kováč L. \& Halse S. (eds) Cave Ecology: 415-434. Springer, Cham.

https://doi.org/10.1007/978-3-319-98852-8

ICZN, International Commission on Zoological Nomenclature 1999. International Code of Zoological Nomenclature (fourth edition). The International Trust for Zoological Nomenclature, c/o The Natural History Museum, London.

Irwin D.E. 2012. A novel approach for finding ring species: look for barriers rather than rings. $B M C$ Biology 10: 21. https://doi.org/10.1186/1741-7007-10-21

Isaac N.J.B., Mallet J. \& Mace G.M. 2004. Taxonomic inflation: its influence on macroecology and conservation. Trends in Ecology and Evolution 19: 464-469. https://doi.org/10.1016/j.tree.2004.06.004

Klimeš L. 2000. Checklist of harvestmen (Opiliones) of Czechia and Slovakia. Ekológia Bratislava 19, Suppl. 3: 125-128.

Koch C.L. 1836. Die Arachniden getreu nach der Natur abgebildet und beschrieben. Vol. 3. C.H. Zeh'sche Buchhandlung, Nürnberg.

Komposch C. 1999. Rote Liste der Weberknechte Kärntens (Arachnida: Opiliones). In: Rottenburg T., Wieser Ch., Mildner P. \& Holzinger W.E. (eds): Rote Listen gefährdeter Tiere Kärntens. Naturschutz in Kärnten 15: 547-565. Amt der Kärntner Landesregierung, Abt. 20, Landesplanung, Klagenfurt.

Komposch C. 2002. The harvestmen fauna of Hungary (Arachnida, Opiliones). In: Samu F. \& Szinetár Cs. (eds) Proceedings of the $20^{\text {th }}$ European Colloquium of Arachnology: 227-242. Szombathely.

Komposch C. 2009. Opiliones (Weberknechte). In: Rabitsch W. \& Essl F. (eds) Endemiten - Kostbarkeiten in Österreichs Pflanzen- und Tierwelt: 476-500. Naturwissenschaftlicher Verein für Kärnten \& Umweltbundesamt.

Komposch C. 2011. Endemic harvestmen and spiders of Austria (Arachnida: Opiliones, Araneae). Arachnologische Mitteilungen 40: 65-79. https://doi.org/10.5431/aramit4008

Komposch C. \& Gruber J. 2004. Die Weberknechte Österreichs (Arachnida, Opiliones). Denisia 12, zugleich Kataloge der Österreichishen Landesmuseen Neue Serie 14: 485-534. 
Kovač I. 1997. Suhe južine (Opiliones) v različnih habitatih v Prekmurju. (Harvestmen (Opiliones) in various habitats in Prekmurje.) Diploma work, Department of Biology, Faculty of Education, University of Maribor.

Kurt K. 2014. Updated checklist of harvestmen (Arachnida, Opiliones) in Turkey. Archives of Biological Sciences 66 (4): 1617-1631. https://doi.org/10.2298/ABS1404617K

Loksa I. 1962. Beiträge zur Kenntnis der Weberknecht-Fauna Ungarns, I. Zoologischer Anzeiger 168: 256-269.

Mallet J. 2013. Subspecies, semispecies, superspecies. In: Levin A.S. (ed.) Encyclopedia of Biodiversity 7: 46-48. Elsevier, Academic Press, San Diego. http://doi.org/10.1016/B978-0-12-384719-5.00138-6

Mammola S., Giachino P.M., Piano E., Jones A., Barberis M., Badino G. \& Isaia M. 2016. Ecology and sampling techniques of an understudied subterranean habitat: the Milieu Souterrain Superficiel (MSS). The Science of Nature 103: e88. https://doi.org/10.1007/s00114-016-1413-9

Marcellino I. 1973. Notizie su Opilioni (Arachnida) italiani e dell'Alto Isonzo. Annali del Museo Civico di Storia Naturale di Genova 79: 192-205.

Martens J. 1978. Spinnentiere, Arachnida: Weberknechte, Opiliones. In: Senglaub F., Hannemann H. \& Schumann H. (eds) Die Tierwelt Deutschlands 64: 1-464. Fischer Verlag, Jena.

Martens J. 2006. Weberknechte aus dem Kaukasus (Arachnida, Opiliones, Nemastomatidae). Senckenbergiana Biologica 86 (2): 145-210.

Miralles A., Vasconcelos R., Perela A., Harris D.J. \& Carranza S. 2010. An integrative taxonomic revision of the Cape Verdean skinks (Squamata, Scincidae). Zoologica Scripta 10: 1-29.

https://doi.org/10.1111/j.1463-6409.2010.00453.x

Monahan W.B., Pereira R.J. \& Wake D.B. 2012. Ring distributions leading to species formation: a global topographic analysis of geographic barriers associated with ring species. BMC Biology 10: e20.

https://doi.org/10.1186/1741-7007-10-20

Mučalica M. 1988. A contribution to the study of harvestmen (Opiliones, Arachnida) on the mountains Avala and Fruska gora (Serbia, Yugoslavia). Glasnik Prirodnjačkog muzeja srpske zemlje. Serija B, Biološke nauke 43/44: 193-198.

Murphree C.S. 1988. Morphology of the dorsal integument of ten opilionid species. Journal of Arachnology 16 (2): 237-252.

Novak T. 2004a. Harvestmen of the Museo Friulano di Storia Naturale in Udine (Arachnida: Opiliones). Part 1. Gortania 26: 211-241.

Novak T. 2004b. An overview of harvestmen (Arachnida: Opiliones) in Croatia. Natura Croatica 13: 231-296.

Novak T. 2005a. An overview of harvestmen (Arachnida: Opiliones) in Bosnia and Herzegovina. Natura Croatica 14: 301-350.

Novak T. 2005b. The harvestmen fauna (Arachnida: Opiliones) from the Sub Mediterranean region of Slovenia - II. Annales, Series historiae naturalis 15: 103-114.

Novak T. 2005c. Terrestrial fauna from cavities in Northern and Central Slovenia, and a review of systematically ecologically investigated cavities. Acta Carsologica 34: 169-210.

https://doi.org/10.3986/ac.v34i1.285

Novak T. \& Gruber J. 2000. Remarks on published data on harvestmen (Arachnida: Opiliones) from Slovenia. Annales, Series historiae naturalis 10: 281-308.

Novak T., Gruber J. \& Slana L. 1984. Remarks on Opiliones from cavities in Slovenia (Yugoslavia). Mémoires de Biospéologie 11: 185-197. 
Novak T., Gruber J. \& Slana L. 1995. A contribution to the knowledge of the harvestmen (Opiliones) from the submediterranean region of Slovenia. Annales, Series historiae naturalis 5: 181-192.

Novak T., Gruber J. \& Slana L. 1996. Weberknechte (Opiliones) des Zentral-Europäischen zoogeographischen Gebietes Sloweniens. Znanstvena revija, Maribor 7: 47-60.

Novak T., Slana L., Červek N., Mlakar M., Žmaher N. \& Gruber J. 2002. Harvestmen (Opiliones) in human settlements of Slovenia. Acta Entomologica Slovenica 10: 131-154.

Novak T., Lipovšek Delakorda S. \& Slana L. 2006. A review of harvestmen (Arachnida: Opiliones) in Slovenia. Zootaxa 1325 (1): 267-276. https://doi.org/10.11646/zootaxa.1325.1.17

Novak T., Janžekovič F., Rak M., Ivajnšič D., Kozel P., Slana Novak L. \& Škornik S. 2017. Can highland habitat type distributions replace mapping of harvestman species? Journal of Insect Conservation 21 (2): 331-343. https://doi.org/10.1007/s10841-017-9979-x

Oatley G., Bowie R.C.K. \& Crowe T.M. 2011. The use of subspecies in the systematics of southern African white-eyes: historical entities or eco-geographic variants. Journal of Zoology 284: 21-30.

https://doi.org/10.1111/j.1469-7998.2010.00772.x

Patten M.A. \& Unitt P. 2002. Diagnosability versus mean differences of sage sparrow subspecies. The Auk 119 (1): 26-35. https://doi.org/10.1093/auk/119.1.26

Raspotnig G., Leutgeb V., Schaider M. \& Komposch C. 2010. Naphthoquinones and anthraquinones from scent glands of a dyspnoid harvestman, Paranemastoma quadripunctatum. Journal of Chemical Ecology 36: 158-162. https://doi.org/10.1007/s10886-010-9745-y

Raspotnig G., Bodner M., Schäffer S., Koblmüller S., Schönhofer A. \& Karaman I. 2014. Chemosystematics in the Opiliones (Arachnida): a comment on the evolutionary history of alkylphenols and benzoquinones in the scent gland secretions of Laniatores. Cladistics 30: 1-8. https://doi.org/10.1111/cla.12079

Raspotnig G., Schaider M., Föttinger P. \& Schönhofer A. 2017. A model for phylogenetic chemosystematics: Evolutionary history of quinones in the scent gland secretions of harvestmen. Frontiers in Ecology and Evolution 5: 139. https://doi.org/10.3389/fevo.2017.00139

Roewer C.F. 1914. Die Familien der Ischyropsalidae und Nemastomatidae der Opiliones-Palpatores. Archives für Naturgeschichte 80 A (3): 99-169.

Roewer C.F. 1917. Über Nemastomatiden und ihre Verbreitung. Archives für Naturgeschichte 83 A (2): $140-160$.

Roewer C.F. 1923. Die Weberknechte der Erde. Gustav Fischer, Jena.

Roewer C.F. 1951. Über Nemastomatiden. Weitere Weberknechte XVI. Senckenbergiana 32: 95-153.

Sangster G. 2009. Number of bird species result from taxonomic progress, not taxonomic inflation. Proceedings of the Royal Society B 276 (1670): 3185-3191. https://doi.org/10.1098/rspb.2009.0582

Schaider M., Novak T., Komposch C., Leis H.-J. \& Raspotnig G. 2018. Methyl-ketones in the scent glands of Opiliones: a chemical trait of cyphophthalmi retrieved in the dyspnoan Nemastoma triste. Chemoecology 28: 61-67. https://doi.org/10.1007/s00049-018-0257-5

Schönhofer A.L. 2013. A taxonomic catalogue of the Dyspnoi Hansen and Sørensen, 1904 (Arachnida: Opiliones). Zootaxa 3679 (1): 1-68. https://doi.org/10.11646/zootaxa.3679.1.1

Schönhofer A.L. \& Holle T. 2007. Nemastoma bidentatum (Arachnida: Opiliones: Nemastomatidae): neu für Deutschland und die Tschechische Republik. Arachnologische Mitteilungen 33: 25-30.

https://doi.org/10.5431/aramit3306 
Schulz J.W. \& Pinto-da-Rocha R. 2007. Morphology and functional anatomy. In: Pinto-da-Rocha R., Machado G. \& Giribet G. (eds) Harvestmen: the Biology of Opiliones: 14-61. Harvard University Press, Cambridge MA.

Staręga W. 1976. Weberknechte (Opiliones, excl. Sironidae) Bulgariens. Annales Zoologici 33(18): 287-433.

Šilhavý V. 1956. Sekáči - Opilionidea. In: Fauna ČSR 7. Nakladatelství Československé akademie věd, Praha.

Torstrom S.M., Pangle K.L. \& Swanson B.J. 2014. Shedding subspecies: The influence of genetics on reptile subspecies taxonomy. Molecular Phylogenetics and Evolution 76: 134-143.

https://doi.org/10.1016/j.ympev.2014.03.011

Willemart R.H., Farine J.-P. \& Gnaspini P. 2009. Sensory biology of Phalangida harvestmen (Arachnida, Opiliones): a review, with new morphological data on 18 species. Acta Zoologica 90: 209-227.

https://doi.org/10.1111/j.1463-6395.2008.00341.x

Wolff J.O., García-Hernández S. \& Gorb S.N. 2016. Adhesive secretions in harvestmen (Arachnida: Opiliones). In: Smith A.M. (ed.) Biological Adhesives: 281-301. Springer.

https://doi.org/10.1007/978-3-319-46082-6

Zink R.M. 2004. The role of subspecies in obscuring avian biological diversity and misleading conservation policy. Proceedings of the Royal Society London B 271: 561-564. https://doi.org/10.1098/rspb.2003.2617

Manuscript received:30 April 2021

Manuscript accepted: 24 August 2021

Published on: 28 October 2021

Topic editor: Rudy C.A.M. Jocqué

Desk editor: Radka Rosenbaumová

Printed versions of all papers are also deposited in the libraries of the institutes that are members of the EJT consortium: Muséum national d'histoire naturelle, Paris, France; Meise Botanic Garden, Belgium; Royal Museum for Central Africa, Tervuren, Belgium; Royal Belgian Institute of Natural Sciences, Brussels, Belgium; Natural History Museum of Denmark, Copenhagen, Denmark; Naturalis Biodiversity Center, Leiden, the Netherlands; Museo Nacional de Ciencias Naturales-CSIC, Madrid, Spain; Real Jardín Botánico de Madrid CSIC, Spain; Zoological Research Museum Alexander Koenig, Bonn, Germany; National Museum, Prague, Czech Republic. 